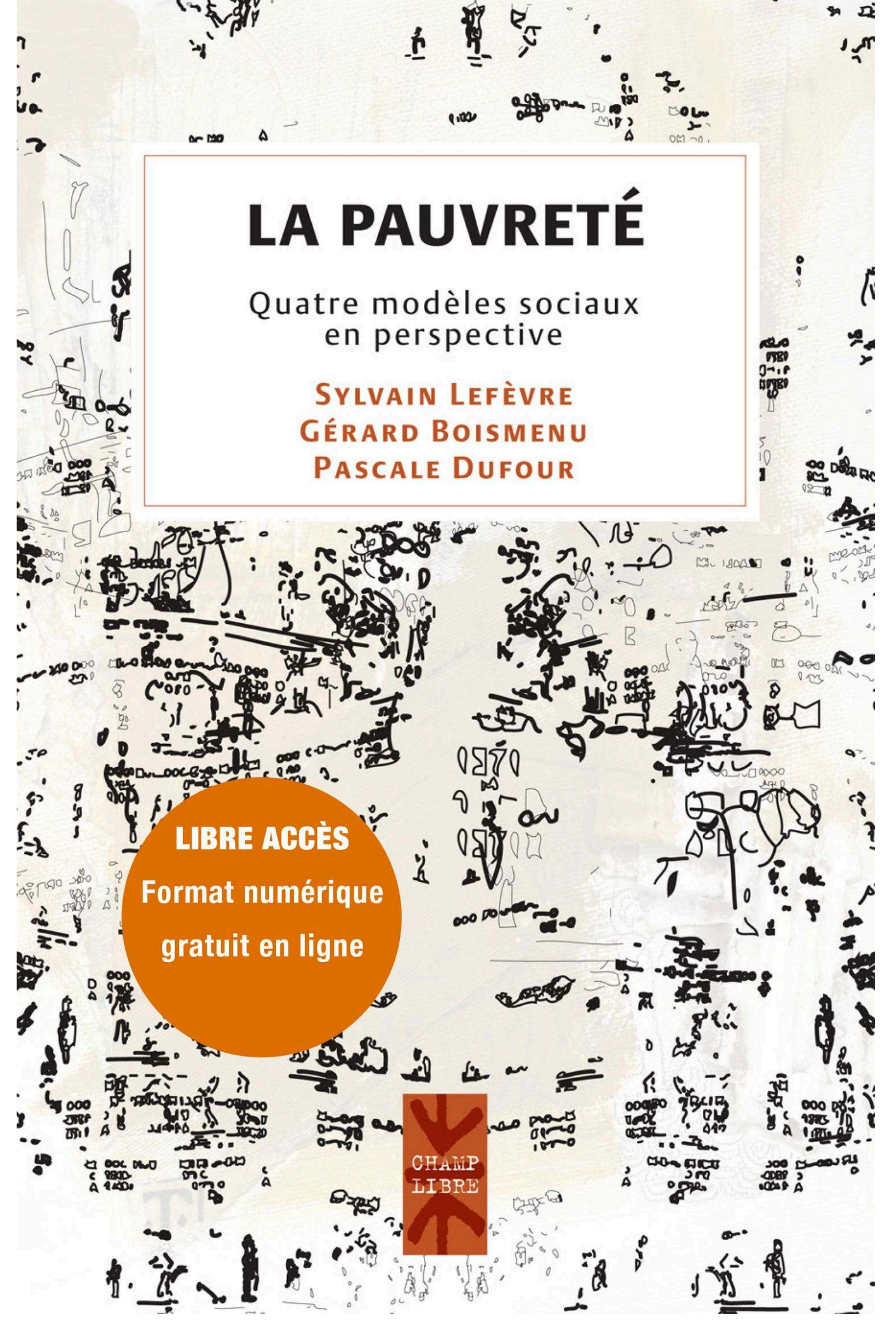




\section{La pauvreté}

Quatre modèles sociaux en perspective

\section{Gérard Boismenu, Pascale Dufour et Sylvain Lefèvre}

DOI : 10.4000/books.pum.4399

Éditeur: Presses de l'Université de Montréal

Lieu d'édition : Montréal

Année d'édition : 2011

Date de mise en ligne : 23 janvier 2018

Collection : Champ libre

EAN électronique : 9782821897632

\section{Q OpenEdition}

\section{Books}

https://books.openedition.org

\section{Édition imprimée}

EAN (Édition imprimée) : 9782760626645

Nombre de pages : 212

Référence électronique

BOISMENU, Gérard ; DUFOUR, Pascale ; et LEFÈVRE, Sylvain. La pauvreté : Quatre modèles sociaux en perspective. Nouvelle édition [en ligne]. Montréal : Presses de l'Université de Montréal, 2011 (généré le 07 décembre 2022). Disponible sur Internet : <http://books.openedition.org/pum/4399>. ISBN :

9782821897632. DOI : https://doi.org/10.4000/books.pum.4399. 


\section{RÉSUMÉS}

La pauvreté traverse l'histoire contemporaine. Elle continue d'exister entre les crises et leur survit. La conjoncture économique ne fait que moduler son ampleur et ses formes d'expression. C'est pourquoi, pour comprendre le phénomène, il faut le situer dans le modèle de développement des sociétés. Pour les auteurs de ce livre en effet, la pauvreté n'est rien de moins que le résultat d'un arrangement politique et social propre à chaque modèle de développement. En d'autres termes, par les formes institutionnelles qu'elles se donnent, les sociétés produisent une pauvreté qui leur ressemble. C'est ce que révèle la comparaison de quatre modèles de l'État social : la Grande-Bretagne, le Danemark, la France et le Québec. Se distancer de l'actualité immédiate tout inquiétante qu'elle soit - c'est peut-être la meilleure manière d'affronter une réalité inconfortable et d'agir en conséquence. 



\section{LA PAUVRETÉ QUATRE MODÈLES SOCIAUX EN PERSPECTIVE}





\section{SYLVAIN LEFÈVRE GÉRARD BOISMENU \\ PASCALE Dufour}

\section{LA PAUVRETÉ}

Quatre modèles sociaux en perspective

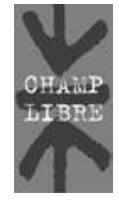


Catalogage avant publication de Bibliothèque et Archives nationales du Québec et Bibliothèque et Archives Canada

Lefèvre, Sylvain

La pauvreté: quatre modèles sociaux en perspective

(Champ libre)

ISBN 978-2-7606-2255-5

1. Pauvreté. 2. Pauvreté - Europe. 3. Pauvreté - Québec (Province).

4. Inégalité sociale. 5. État providence.

I. Boismenu, Gérard. II. Dufour, Pascale, 1971- . III. Titre.

IV. Collection: Champ libre (Presses de l'Université de Montréal).

HC79.P6L43 $2011 \quad 339.4$ '6 C2O11-9406O6-3

ISBN (version imprimée) 978-2-7606-2255-5

ISBN (version numérique PDF) 978-2-7606-2664-5

Dépôt légal: $2^{\mathrm{e}}$ trimestre 2011

Bibliothèque et Archives nationales du Québec

(C) Les Presses de l’Université de Montréal, 2011

Les Presses de l'Université de Montréal reconnaissent l'aide financière du gouvernement du Canada par l'entremise du Fonds du livre du Canada pour leurs activités d'édition.

Les Presses de l'Université de Montréal remercient de leur soutien financier le Conseil des Arts du Canada et la Société de développement des entreprises culturelles du Québec (SODEC). 


\section{AVANT-PROPOS}

Depuis les évènements financiers dramatiques de 2008, de nombreuses analyses circulent qui font état de reculs sociaux importants en Europe et en Amérique du Nord. Les taux de chômage à la hausse, les banqueroutes des personnes et des ménages, les fermetures d'entreprises, remettent la pauvreté à l'ordre du jour des médias et des gouvernements. Cet ouvrage est, en partie, une réaction à ces discours «à chaud» à propos de la pauvreté. La pauvreté avait-elle réellement «disparue» avant la crise et est-elle uniquement une question de conjoncture économique? Nous avons fait le choix éditorial de nous en remettre à une analyse historique afin de contrecarrer les multiples effets d'annonce, que ce soit les grands programmes politiques ou les outils conventionnels de classement, de type PNB. En partant des données structurelles qui offrent un regard plus en profondeur sur la question de la pauvreté (répartition des richesses, types de pauvreté, segments précarisés de la population), nous espérons présenter une image plus juste des transformations en cours.

De même, il nous paraît important de ne pas considérer la pauvreté et les inégalités comme une donnée externe aux 
sociétés - rôle que peuvent jouer, par exemple, les chocs économiques -, qui nécessiterait des réponses politiques, de court terme. En renversant la perspective, et en partant des sociétés, nous avons voulu souligner que les compromis politiques, sociaux et économiques qui structurent ces sociétés «produisent» aussi certaines formes de pauvreté.

Par ailleurs, nous articulons des enseignements tirés de l'analyse économique, sociologique et politique afin de présenter une analyse la plus complète possible (bien que non exhaustive) des différentes dimensions de la pauvreté.

\section{Pourquoi lire ce livre?}

La position développée au fil des chapitres s'articule autour de trois propositions:

1. Nous considérons les différentes formes de pauvreté comme le résultat des arrangements politiques et institutionnels de chaque société. Bien sûr la pauvreté est aussi une question de macroéconomie et s'articule à des données plus sectorielles (par exemple, le marché du logement), mais ici, nous considérons la médiatisation de cette question par les arrangements institutionnels dans les milieux de travail, de l'emploi, des mesures sociales, au sein des États, pour montrer comment ces architectures sociopolitiques influencent la production de la pauvreté et la manière dont les sociétés la traitent.

2. Nous proposons une analyse comparée qui tente d'aller au-delà des archétypes. Quand on sort des «canons» de la comparaison dans le champ de l'analyse des États sociaux, pour se pencher davantage sur des cas hybrides, qu'apprenonsnous? Nous verrons que conclure à une convergence des États n'est pas une conclusion valide selon notre grille d'analyse.

3. En nous questionnant sur le sens des transformations en cours, nous mettons à profit cette comparaison, en illus- 
trant, notamment dans la dernière partie de l'ouvrage, comment une analyse plus fine permet de saisir le travail distinct à l'œuvre dans les sociétés. Pauvreté et inégalités sont présentes partout, mais le sens qu'elles revêtent socialement (et sociologiquement) diffère beaucoup; même si a priori la distance entre les sociétés diminue.

\section{Comment le lire?}

L'ouvrage est construit en trois temps. Le premier chapitre revient sur les principales analyses des formes différenciées de pauvreté suivant les sociétés. Nous mettons en relief les principaux apprentissages, de même que les limites de ces travaux, en revenant notamment sur les difficultés liées à la mesure même de la pauvreté. Pour bien saisir comment la question de la pauvreté s'inscrit dans des configurations institutionnelles propres à chaque société, nous proposons une analyse de ses fondements autour de quatre piliers:

- le marché du travail et ses régulations,

- les compromis sociaux entre les acteurs syndicaux, économiques et étatiques,

- le traitement politique réservé aux personnes pauvres,

- les représentations sociales de la pauvreté.

Ces réflexions nous ont conduits à penser la comparaison, qui constitue le deuxième temps de l'ouvrage, en deux «morceaux» distincts. Les chapitres 2 et 3 comparent deux archétypes bien connus de la comparaison des régimes d'État social: le Danemark et la Grande-Bretagne, alors que les chapitres 4 et 5 explorent des cas hybrides, correspondant moins directement aux canons de la comparaison entre la France et le Québec. Cette sortie des sentiers battus met en relief la complexité des processus qui sous-tendent la pauvreté et les inégalités sociales pour chaque société. Finalement, le troisième temps (chapitres 6 et 7) est consacré à un regard croisé 
sur les quatre cas du point de vue de la convergence supposée des États sociaux. En partant d'une discussion critique des indicateurs de la comparaison en matière de pauvreté et d'inégalités, nous revisitons cette question de la convergence en proposant non plus de partir des politiques sociales nationales, mais bien des formes différenciées de pauvreté et d'inégalités au sein de chaque espace social. 


\section{Comprendre la pauvreté: enjeux politiques et analytiques}

La crise financière et économique de la deuxième moitié des années 2000 suscite des conséquences sociales dans de nombreux pays de l'OCDE, avec une augmentation dramatique des taux de chômage. Les conséquences sont aussi politiques, avec un apparent «retour de l'État» dans la gouvernance économique. Les gouvernements déplorent un dévoiement de l'économie de marché par le biais de la financiarisation du capitalisme, et appellent à la fois à un retour à la dimension «productive» du système («l'économie réelle» contre les «bulles spéculatives») et à une reprise en main de l'État sur l'encadrement de l'économie. Les difficultés actuelles découleraient en effet des excès du «laisser-aller» (dans un sens péjoratif) d'acteurs économiques influents: cupidité des courtiers, complaisance des responsables bancaires, indécence des bénéficiaires de «parachutes dorés»...

$\mathrm{Au}$-delà de cette rhétorique moralisatrice, les dirigeants politiques affrontent la crise économique actuelle en proclamant également le retour de l'État, y compris dans son rôle d'encadrement de l'activité économique. À ce titre, des mesures présentées auparavant comme contraires au bon fonctionnement de l'économie de marché sont promues par les 
décideurs politiques et économiques: politique de relance budgétaire, baisse des taux d'intérêt, nationalisation, réglementation des institutions financières, voire mesures protectionnistes. Qu'on assiste à un véritable tournant dans l'orientation des politiques sociales et économiques ou que cette séquence ne soit qu'un intermède afin de replâtrer un édifice lézardé, seul le temps permettra d'en juger.

\section{La permanence de la pauvreté}

Une prise de recul historique invite à la prudence dans l'appréciation de la situation contemporaine. D'une part, la «question sociale» (que ce soit l'accroissement des inégalités ou la persistance de la pauvreté) n'avait pas disparu ces dernières décennies, y compris durant les années de croissance. D'autre part, les politiques de «laisser-faire» et de déréglementation financière et économique ne doivent pas conduire à survaloriser l'autonomie de la sphère économique. Concrètement, les incidences sociales des systèmes économiques des pays de l'OCDE sont intimement liées aux décisions politiques et aux arbitrages au sein des sociétés.

Un rapport de l'OCDE, paru à l'automne 2008, permet d'appuyer ces deux éléments ${ }^{1}$. Comme son titre l'indique, Croissance et inégalités, le document met l'accent sur l'accroissement depuis le milieu des années 1980 des inégalités sociales, malgré des années placées sous le signe de la croissance économique.

Le cas des États-Unis est emblématique à la fois de la désarticulation des notions de croissance et de partage de la richesse, et des effets d'optique liés à la focalisation sur quelques indicateurs fétiches, comme le PNB (produit national

1. OCDE, Croissance et inégalités: Distribution des revenus et pauvreté dans les pays de l'OCDE, octobre 2008. 
brut) et le taux de croissance. C'est aujourd'hui le pays le plus riche en termes de production par habitant: parmi treize pays développés, si l'on associe un indice 100 aux États-Unis pour le PNB par habitant, la position relative des autres pays va de 85 (Suisse) à 65 (France), avec un indice moyen de $76^{2}$. Pour autant, on ne peut en déduire que la population américaine est la plus riche, si tant est que l'on prend en compte la répartition de cette richesse. L'étude comparée de la distribution du revenu monétaire disponible, après transferts sociaux et impôt positif et négatif, révèle qu'il n'en est rien, ce qui va à l'encontre de l'intuition portée par le sens commun.

Alors que durant les décennies qui ont suivi la Deuxième Guerre mondiale, la croissance de la production et de la productivité avait donné une impulsion à la classe moyenne américaine et avait été accompagnée par un resserrement de la distance économique entre les mieux et les plus mal nantis, il apparaît que de 1973 à 2006 (c'est-à-dire avant le déclenchement de la «crise des subprimes»), la tendance s'inverse. Durant cette séquence temporelle, le PNB a crû aux ÉtatsUnis de plus de $85 \%$ et la productivité a augmenté de près de $50 \%$, mais l'inégalité est revenue à des niveaux inconnus depuis la crise des années $1930^{3}$.

L'inégalité des revenus n'est donc pas affaire de rareté de biens; elle peut aller de pair avec un développement économique soutenu, ce qui est d'ailleurs une caractéristique forte de la société américaine contemporaine. Si l'on considère l'écart entre les $20 \%$ les plus fortunés et les $20 \%$ les moins dotés aux États-Unis, on observe qu'après un resserrement

2. Timoty S. Smeeding, Lee Rainwater, Comparing Living Standards across Nations: Real Incomes at the Top, the Bottom, and the Middle, University of New South Wales, 2002.

3. Shawn Fremstad et al., Movin' On Up: Reforming America's Social Contract to Provide a Bridge to the Middle Class, Centre for Economic Policy Research, 2008. 
tout relatif de 1965 à 1980, la bipolarisation s'exacerbe fortement depuis. Alors que les plus faibles revenus font quasiment du surplace, les autres quintiles voient leur part de la richesse s'accentuer, de manière de plus en plus prononcée à mesure qu'on s'approche du sommet. D'ailleurs, c'est pour le $1 \%$ des familles les plus riches que la poussée est la plus fulgurante ${ }^{4}$.

L'accentuation des inégalités n'est pas l'apanage des ÉtatsUnis. Non seulement on assiste à l'approfondissement des inégalités dans la plupart des pays de l'OCDE, alors que la production générale de richesses s'accroît, mais également à la persistance de la pauvreté, comme nous le verrons dans cet ouvrage. Toutefois, cette pauvreté touche des segments de population différents selon les configurations nationales. C'est là le second point à mettre en relief: au-delà des performances mesurées par tel ou tel indicateur (le nombre de personnes pauvres, le revenu moyen, etc.), ce ne sont pas les mêmes groupes qui sont touchés selon les pays, et ils ne font pas face aux mêmes dispositifs de politiques publiques. Derrière le consensus néolibéral apparemment partagé par les différents gouvernements depuis trois décennies, avec plus ou moins de volontarisme et d'enthousiasme, coexistent donc des modèles sociaux différents. Par ce terme, on entend l'architecture des interdépendances sociales au sein d'une société, «ce que se doivent mutuellement les citoyens et la manière dont sont organisées lesdites obligations ${ }^{5}$ ».

4. Sherman Arloc, Income Inequality Hits Record Levels, New CBO Data Show - Incomes Rose \$180,00o for Top One Percent in 2005. But Just \$400 for MiddleIncome Households, Center on Budget and Policy Priorities, 2007.

5. George Ross, «Les modèles sociaux dans le maelström: l'avenir de la citoyenneté sociale dans l'Union européenne», Jane Jenson, Bérengère Marques-Pereira, Éric Remacle (dir.), L'état des citoyennetés en Europe et dans les Amériques, Montréal, Presses de l’Université de Montréal, 2007, p. 355. 
On ne saurait aborder la pauvreté et les inégalités comme un «raté», plus ou moins inattendu; il faut y voir davantage le produit d'arbitrages politiques et de configurations socioéconomiques, autrement dit de «choix de société». Il ne s'agit pas d'imputer quelque caractère intentionnel, planifié et maîtrisé à cette production de la pauvreté, que ce soit de la part des élites politiques et économiques d'un pays, ou de ses habitants. Néanmoins, pour provocatrice qu'elle puisse sembler, cette formule a le mérite de «tordre le bâton dans l'autre sens» par rapport aux visions de la pauvreté et des inégalités fatalistes («il y en a toujours eu» et «il faut bien des perdants»), ou techniciste (c'est un problème temporaire qui sera résorbé quand le bon réglage socioéconomique sera trouvé).

Les études de cas permettent de montrer comment les sociétés, par les politiques publiques mises en place et les choix opérés en termes de redistribution des richesses, s'accommodent, d'une certaine façon et chacune à leur manière, de la pauvreté.

\section{Transformations des trajectoires de l'État social}

Cette approche nous permet de revenir, dans la dernière partie de l'ouvrage, sur le débat concernant la convergence ou la différenciation des États sociaux. Pour résumer ce débat, la première position diagnostique un alignement généralisé des politiques économiques et sociales à travers les pays. Le plus souvent, ce processus est lié à des bouleversements macrosociaux exogènes (mondialisation, vieillissement de la population, société postindustrielle) ou aux limites endogènes de l'État social (crise financière, de légitimité, d'efficacité) tels qu'ils auraient prévalu dans de nombreux pays occidentaux dans la seconde moitié $\mathrm{du} \mathrm{XX}^{\mathrm{e}}$ siècle. 
Ce constat prend parfois des tonalités normatives, comme dans la plupart des travaux de l'OCDE, où la «nécessaire réforme» et l'incontournable «modernisation» correspondent à un vade-mecum désormais bien connu, ou dans les discours des opposants au néolibéralisme où la vague néolibérale est présentée comme faisant tomber les digues sociales. Dans les deux cas, le constat est le même: on assisterait depuis le début des années 1980 à un aplanissement des différences, via une «course vers le bas» collective, entre les différents projets sociaux des pays.

Dans ce débat, la position contraire insiste sur l'irréductible différence entre les régimes d'État social. Cette différence a été schématisée par des typologies assez contrastées. Avec sa typologie qui a fait école, Esping-Andersen distingue notamment le degré de «démarchandisation» que les États autorisent, entre le régime libéral, le régime conservateurcorporatiste et le régime social-démocrate ${ }^{6}$. Elle a fait l'objet de nombreuses critiques ${ }^{7}$; outre celles provenant des études féministes $^{8}$, la plupart contestent l'affiliation de tel ou tel pays, quelques-unes proposent la formalisation de régimes complémentaires (méditerranéen-familialiste ${ }^{9}$ ou des antipodes $^{10}$ ). Mais ces développements critiques renforcent in fine

6. Gøsta Esping-Andersen, The Three Worlds of Welfare Capitalism, Princeton University Press, 1990.

7. Pour une synthèse, voir François-Xavier Merrien, «États-providence en devenir. Une relecture critique des recherches récentes», Revue française de sociologie, vol. 43, $\mathrm{n}^{\circ}$ 2, 2002, p. 211-242.

8. Jane Lewis, «Gender and the development of welfare regims», Journal of European Social Policy, vol. 2, n 3, 1992, p. 159-173; A. S. Orloff, «Gender and the social rights of citizenship : the comparative analysis of gender relations and welfare states», American Sociological Review, vol. 58, n 3, 1993, p. 303-328; D. Sainsbury (dir.), Gendering Welfare States, Sage Publications, 1994.

9. Maurizio Ferrera, «The southern model of welfare in social Europe», Journal of European Social Policy, vol. 6, $\mathrm{n}^{\circ}$ 1, 1996, p. 17-37.

10. F. G. Castles, M. Mittchell, «Worlds of welfare and families of nations », in F. G. Castles (dir.), Families of Nations: Patterns of Public Policy in Western Democracies, Darmouth, 1993. 
une tendance au durcissement théorique de familles hétérogènes de régimes. On voit mal la compatibilité de cette approche avec celle qui met l'accent sur l'homogénéisation tendancielle des régimes.

Une seconde opposition caractérise ce débat sur les évolutions des politiques sociales au sein de pays de l'OCDE. Contre la perspective mettant l'accent sur la diffusion internationale, voire l'hégémonie des réformes néolibérales, à partir du début des années 1980, des travaux invitent notamment à considérer avec davantage de retenue les emblématiques réductions des dépenses sociales. L'étude de Paul Pierson insiste sur l'incapacité des gouvernements néolibéraux de M. Thatcher en Angleterre et de R. Reagan aux États-Unis à mener leurs projets de reconfiguration radicale des politiques sociales ${ }^{11}$. Outre le poids des institutions et des politiques héritées du passé (notion de «path dependance»), l'auteur souligne à la fois l'impopularité de ce type de réformes et la résistance de multiples groupes sociaux ayant développé des intérêts objectifs dans l'existence de l'État social.

Si l'hypothèse d'un tournant radical semble invalidée, il ne faut pas en conclure que tout changement est impossible; il prend plutôt les traits de modifications incrémentales, limitées, et produisant leurs effets avec une temporalité différée. Surtout, les changements possibles sont ceux qui s'ancrent paradoxalement dans une certaine continuité, au moins apparente, avec les logiques institutionnelles héritées.

Cette réflexion sur les dynamiques de reproduction et d'altération de logiques instituées croise les deux axes du débat entre, d'une part, l’opposition caractérisée par l'«homogénéisation des pratiques» et par l'«essentialisation de régimes

11. Paul Pierson, Dismantling the Welfare State? Reagan, Thatcher and the Politics of Retrenchment, Cambridge University Press, 1995. 
d'État social distincts» et, d'autre part, l'opposition du «tournant néolibéral hégémonique» face à la «résistance des logiques institutionnelles». Pour articuler ces différentes problématiques, il s'agit d'analyser les transformations récentes des régimes d'État social, et notamment les problèmes communs qu'ils affrontent, en mettant l'accent sur les réponses différenciées qu'ils y apportent, en fonction de leurs forces et handicaps structurels ${ }^{12}$.

Face à la diffusion ces dernières années de recettes relativement standardisées, souvent désignées par le terme de workfare (activation des dépenses sociales, développement de la conditionnalité des aides, notion d'investissement social ${ }^{13}$ ), les déclinaisons nationales adoptées sont de bons traceurs et révélateurs des configurations différenciées. Les manières dont sont appropriés tels ou tels dispositifs, l'«adoucissement» ou au contraire le «durcissement» d'une contrainte, l'euphémisation ou au contraire l'accent mis sur une mesure, sont autant de variations au sein desquelles se négocient des compromis institutionnels et politiques complexes. De même, s'intéresser aux visages différenciés de la pauvreté et des inégalités selon les pays nous renseigne sur la trajectoire de ces régimes, la forme des compromis socioéconomiques qui s'y nouent. À rebours de la plupart des démarches qui questionnent l'évolution des États sociaux «par le haut», c'est-à-dire en étudiant les façades institutionnelles et les directions politiques proclamées, nous pro-

12. Gøsta Esping-Andersen (dir.), Welfare States in Transition: National Adaptation in Global Economies, Sage Publications, 1996; Paul Pierson (dir.), The New Politics of the Welfare State, Oxford University Press, 2001.

13. Pascale Dufour, Gérard Boismenu, Alain Noël, L'aide au conditionnel. La contrepartie dans les mesures envers les personnes sans emploi en Europe et en Amérique du Nord, Presses de l’Université de Montréal, 2003; Gøsta EspingAndersen, Trois leçons sur l'État-providence, Seuil, 2008; Jean-Claude Barbier, La longue marche vers l'Europe sociale, PUF, 2009. 
posons une réflexion qui éclaire, «par le bas», les différentes situations de pauvreté selon les pays et les dispositifs dans lesquels elles s'insèrent.

En partant de la classification acceptée des États sociaux, nous avons construit une comparaison qui nous permet de traiter, théoriquement et empiriquement, la question des transformations des trajectoires de l'État social. Premièrement, nous retenons deux cas exemplaires de régimes distincts: le Danemark et la Grande-Bretagne ${ }^{14}$. Le premier, loué pour l'articulation réussie entre flexibilité économique et protection sociale, s'oppose quasiment terme à terme au second, réputé «produire» un nombre croissant de laisséspour-compte, dans le but de ne pas entraver le fonctionnement du marché. Comment ont évolué ces deux modèles, quasi fétichisés dans la littérature, et quels accommodements de la pauvreté proposent-ils?

Nous contrastons cette première comparaison à un second couple, moins standard, le Québec et la France. Prototypes supposés de formes d'hybridation et/ou cas «inclassable», la comparaison enrichie de ces deux cas nous permet de prendre une mesure plus fine du sens des transformations récentes: entre convergence, apprentissages mutuels ou résistances institutionnelles.

L'intérêt de la démarche, on l'aura compris, ne réside pas tant dans la production de nouvelles données par rapport à des cas vus et revus dans la littérature, mais bien davantage, dans la construction d'une comparaison qui nous conduit, dans la deuxième partie de l'ouvrage, à une réflexion plus

14. Dans cet ouvrage, nous traitons principalement de la Grande-Bretagne (Angleterre, Écosse et Pays de Galles). Néanmoins, il sera parfois fait référence au Royaume-Uni (incluant donc l'Irlande du Nord) puisque la plupart des études statistiques, notamment de l'OCDE, s'y réfèrent. 
générale sur la mesure et la lecture que nous faisons des fondements de la pauvreté.

Afin de procéder à des comparaisons significatives entre pays de l'OCDE, il faut nous entendre sur les termes utilisés et sur les instruments de mesure adoptés. Pour cela, nous allons revenir dans un premier temps sur les enjeux d'une «mesure» de la pauvreté, puis sur la méthodologie suivie pour forger une analyse comparée entre plusieurs types de configurations nationales.

\section{Prendre la mesure de la pauvreté}

Circonscrire le phénomène de la pauvreté impose à tout le moins que l'on adopte une définition de la notion. Le repérage quantitatif pose plusieurs problèmes, renforcés par la perspective comparative que nous avons retenue. Il nous faut retenir une définition et un repérage de la pauvreté à la fois féconds et en accord avec la disponibilité des données dans chacun des pays étudiés. Par exemple, il n'y a pas de consensus au Canada sur ce que devrait être la mesure de la pauvreté $^{15}$. Ainsi, suivant les indicateurs retenus, le Québec est présenté comme une province ayant un haut taux de pauvreté ou comme ayant un niveau de pauvreté parmi les plus faibles au pays ${ }^{16}$. De plus, contrairement à la situation qui prévaut au sein des pays européens, où le décompte de la pauvreté a été standardisé, il n'existe pas de seuil officiel de pauvreté. Les débats surgissent régulièrement à ce propos ${ }^{17}$. Il ne faut pas perdre de vue que les indicateurs que l'on peut

15. Gouvernement du Québec, Inventaire des indicateurs de pauvreté et d'exclusion sociale. Conditions de vie, 2005, Introduction.

16. Marie-Renée Roy, Guy Fréchet, Frédéric Savard, «Travail et pauvreté au Canada», Options politiques, IRPP, 2008.

17. Gouvernement du Québec, Inventaire des indicateurs de pauvreté et d'exclusion sociale. Conditions de vie, op. cit. 
retenir sont d'abord des balises, des repères, des indices: c'est une façon de se représenter un phénomène, mais ce n'est pas le phénomène en lui-même. La première ambivalence dans le repérage de ce qu'on nomme «pauvreté» oscille entre les conceptions «absolue» et «relative» du phénomène. Nous devons également préciser deux dimensions centrales de la problématique de la pauvreté: les liens possibles entre le niveau de pauvreté et l'inégalité sociale; les liens de plus en plus complexes entre la pauvreté et l'emploi.

\section{La pauvreté absolue}

La conception objective de la pauvreté fait référence aux ressources économiques (ou matérielles). Au début du $\mathrm{XX}^{\mathrm{e}}$ siècle, Rowntree ${ }^{18}$ propose d'associer la pauvreté à une insuffisance de revenu pour assurer les besoins essentiels; on en vient à parler de la notion de pauvreté absolue. La mesure de la pauvreté absolue est établie en référence au revenu nécessaire permettant à la personne ou au ménage de se procurer les biens essentiels de subsistance basés sur les besoins biologiques, en termes d'aliments, d'eau, de vêtements et d'habitation. On a là le minimum pour maintenir en état l'efficacité physique des individus. De là, la volonté affirmée d'établir scientifiquement les standards minimaux.

Cette grille d'analyse n'est pas sans susciter plusieurs critiques, notamment sur la conception implicite de la pauvreté que cela suppose. Il est difficile de définir ce qu'il faut entendre par le minimum nécessaire pour la reproduction physique de l'individu ou de la famille et d'y associer un niveau de revenu. Il en va de même des standards et besoins qui varient selon les individus, les cultures, les sociétés et,

18. Seebohm Rowntree, Poverty: A Study of Town Life, Nelson, 1901; Seebohm Rowntree, Poverty and Progress, Longmans Green, 1941. 
surtout, dans le temps. Dans cette démarche, ce sont les besoins physiques que l'on prend en compte, alors que les besoins sociaux et culturels sont laissés de côté. En somme, le seuil établi et fondé par la reproduction physique est une vision étriquée de la pauvreté. Durant les années 1960, on en est venu à revoir cette approche et à la dépasser en allant au-delà des besoins physiques et biologiques, et en relevant le caractère multidimensionnel de ce phénomène. La pauvreté a été mise en relation avec le niveau de vie généralement accepté dans une société donnée et à un moment donné. Townsend écrit à ce propos :

Les individus peuvent être considérés comme pauvres quand ils manquent des ressources nécessaires pour obtenir les types de régimes alimentaires, participer aux activités et avoir les conditions de vie générales de la société à laquelle ils appartiennent. Leurs ressources sont tellement en dessous de celles des individus ou des familles moyennes qu'ils sont, dans les faits, exclus des modes ordinaires de consommation et d'activités ${ }^{19}$. (Notre traduction)

Des travaux ont pris acte de ces critiques et ont élargi l'éventail des éléments nécessaires à une couverture des «besoins de base». On peut imaginer, par exemple, la mesure d'un panier de consommation où, en plus de la nourriture, des vêtements et du logement, on inclut le transport et l'accès à certains services jugés indispensables ${ }^{20}$. On ne parle plus seulement de la subsistance des personnes, mais davantage de la répartition et du niveau de ressources nécessaires pour que la personne soit en mesure de participer activement à la vie en société. La formalisation de ce seuil est alors un point

19. Peter Townsend, Poverty in the United Kingdom, A Survey of Household Resources and Standards of Living, Penguin Books \& Allen Lane, 1979, p. 31.

20. Voir les travaux du Centre d'étude sur la pauvreté et l'exclusion, 2009. 
de passage qui permet d'ouvrir sur une conception relative de la pauvreté.

\section{La pauvreté relative}

La pauvreté est relative en ce sens qu'elle est inscrite et posée en relation à la société historiquement datée dans laquelle les personnes vivent. La question centrale devient celle du seuil à partir duquel les personnes sont réputées participer correctement à la vie sociale commune. Par définition, ce seuil se situe à un niveau supérieur à celui de la pauvreté absolue. Mais à quel point exactement? Sur ce plan, les perspectives normatives interfèrent nécessairement puisqu'en désignant un niveau relatif de revenu permettant la participation à la Cité, c'est l'idéal de la citoyenneté qui est débattu.

Au Canada, ce seuil est établi à 50\% de la médiane des revenus disponibles. En Europe, le seuil de 60\% de la médiane du revenu disponible a été adopté. On mesure sans peine l'enjeu du choix de tel ou tel seuil. Ainsi, alors qu'en France, le seuil de pauvreté fut longtemps fixé à 50\% du revenu médian, l'Insee a récemment privilégié le seuil de pauvreté à $60 \%$ du revenu médian afin d'harmoniser son mode de calcul sur celui de l'institut de statistique européen Eurostat. Conséquence immédiate: le taux de pauvreté monétaire a été multiplié par deux ${ }^{21}$. Si, en 2006, en retenant un seuil de pauvreté à $50 \%$ du revenu médian, 7,1\% de la population française était considérée comme pauvre, la proportion passait à 13,2\% en retenant le seuil de $60 \%$ du revenu médian. Nous reviendrons dans la seconde partie de l'ouvrage sur l'importance politique des effets de cadrage et d'affichage

21. Anne Lambert, «Quelle mesure officielle pour la pauvreté?», Regards croisés sur l'économie, vol. 2, no 4, 2008, p. 30-33. 
que permettent tour à tour l'instrumentation et l'instrumentalisation des indicateurs statistiques.

L'établissement d'un seuil de pauvreté relative essuie un certain nombre de critiques. En effet, ce seuil n'est rien d'autre qu'une construction statistique abstraite, qui n'a pas de validité indépendante autre que de donner une indication empirique de la pauvreté. Dans la mesure où ce seuil est fonction de la distribution des revenus (un pourcentage du revenu médian), il témoigne tout autant de l'inégalité des revenus que de la pauvreté en tant que telle. Cette pauvreté relative n'a donc de sens que dans les sociétés prises distinctement, et beaucoup moins quand on juxtapose plusieurs sociétés assez dissemblables (le pauvre en Roumanie est beaucoup plus pauvre que le pauvre en Allemagne, par exemple). Cependant, c'est la mesure la plus féconde et la plus largement disponible pour travailler dans une perspective comparative.

Ainsi, Smeeding, Rainwater et Burtless dressent un tableau permettant de comparer la situation respective de 18 pays au milieu des années $1990^{22}$. Deux seuils sont retenus. Le premier, celui de $40 \%$ du revenu disponible (équivalent) médian, qui correspond en fait au seuil officiel de pauvreté aux ÉtatsUnis et, le deuxième, où la barre est fixée à $50 \%$ de la médiane. Des 18 pays retenus, les taux varient de 1,3\% (Luxembourg) à 10,7\% (aux États-Unis), en prenant le seuil de $40 \%$ de la médiane, alors que la moyenne est de 4,8\%. Les États-Unis, à un bout du spectre, font plus que doubler cette moyenne. Il ne peut nous échapper que 11 pays, de l'Europe continentale et du Nord, sont en deçà de la moyenne des 18 pays. Si l'on prend maintenant le seuil de $50 \%$, toujours pour le milieu des

22. Timothy M. Smeeding, Lee Rainwater, Gary Burtless, «U.S. Poverty in a Cross-national Context», Sheldon Danziger, Robert H. Haveman (dir.), Understanding Poverty, Harvard University Press, 2001. 
TABLEAU 1.1

Taux de pauvreté dans 18 pays au milieu des années 1990, par seuils de pauvreté

\begin{tabular}{|c|c|c|c|c|c|}
\hline \multirow{2}{*}{ Pays } & \multirow[t]{2}{*}{ Année } & \multicolumn{2}{|c|}{$40 \%$ taux de pauvreté } & \multicolumn{2}{|c|}{$50 \%$ taux de pauvreté } \\
\hline & & Taux & Rang & Taux & Rang \\
\hline États-Unis & 1997 & 10,7 & 1 & 17,8 & 1 \\
\hline Italie & 1995 & 8,9 & 2 & 13,9 & 2 \\
\hline Australie & 1994 & 7,0 & 3 & 6,7 & 13 \\
\hline Canada & 1994 & 6,6 & 4 & 11,4 & 4 \\
\hline Royaume-Uni & 1995 & 5,7 & 5 & 13,2 & 3 \\
\hline Espagne & 1990 & 5,2 & 6 & 10,4 & 5 \\
\hline Israël & 1992 & 5,2 & 6 & 10,2 & 6 \\
\hline Pays-Bas & 1994 & 4,7 & 8 & 7,9 & 7 \\
\hline Suède & 1992 & 4,6 & 9 & 6,5 & 15 \\
\hline Allemagne & 1994 & 4,2 & 10 & 7,5 & 8 \\
\hline Suisse & 1992 & 4,0 & 11 & 6,9 & 11 \\
\hline Danemark & 1992 & 3,6 & 12 & 7,1 & 10 \\
\hline France & 1994 & 3,2 & 13 & 7,4 & 9 \\
\hline Norvège & 1995 & 3,0 & 14 & 6,9 & 11 \\
\hline Autriche & 1992 & 2,8 & 15 & 6,7 & 13 \\
\hline Finlande & 1995 & 2,1 & 16 & 5 & 17 \\
\hline Belgique & 1992 & 1,9 & 17 & 5,5 & 16 \\
\hline Luxembourg & 1994 & 1,3 & 18 & 3,9 & 18 \\
\hline $\begin{array}{l}\text { Moyenne pour } \\
\text { l'ensemble }\end{array}$ & & 4,7 & & 8,6 & \\
\hline
\end{tabular}

Source: Timothy M. Smeeding, Lee Rainwater, Gary Burtless, «U.S. Poverty in a Crossnational Context », Sheldon Danziger, Robert H. Haveman (dir.), Understanding Poverty, Harvard University Press, 2001, p. 186 (notre traduction).

années 1990, le taux moyen de pauvreté passe de 4,8 à 8,6\%. Il y a toujours un écart considérable entre les deux pôles et, plutôt que d'y voir une progression linéaire d'un pays à l'autre, il y a davantage une cassure avec, d'un côté, surtout des pays anglo-saxons et, de l'autre, les pays d'Europe continentale et d'Europe scandinave. De façon générale, que l'on retienne l'un des seuils ou l'autre, la position relative des pays change peu. La seule réserve concerne la très nette amélioration de la 
performance de l'Australie et de la Suède en retenant le deuxième seuil, témoignant de sociétés plus égalitaires.

Plus près de nous, soit au tournant des années 2000, les données pour huit pays témoignent d'écarts toujours importants entre les taux de pauvreté ${ }^{23}$. Avec le seuil de $50 \%$ de la médiane du revenu disponible, la Finlande est à 5,4\%, alors que les États-Unis sont à $17 \%$, et la moyenne se situe à 9,8\%. Si on opère des découpages par âge (enfants, aînés, foyer monoparental, sans enfants, etc.), mis à part dans la catégorie «adultes sans enfants» (ils y occupent la deuxième place), les États-Unis ont le pire taux de pauvreté dans chaque catégorie. Dans tous les types de ménage, les États-Unis ont un taux de pauvreté supérieur à la moyenne; de façon régulière, c'est le Canada ou le Royaume-Uni qui arrive au deuxième rang.

La forte inégalité des salaires joue un rôle primordial dans le taux de pauvreté basé sur une relation au revenu disponible. À l'inverse, les pays avec une structure plus égalitaire des salaires ont des taux de pauvreté relative moins élevés. Ce lien entre pauvreté et inégalités n’est pas linéaire, mais il se vérifie dans la concordance des classements des pays sur ces deux échelles (les pays les plus inégalitaires étant aussi les pays où les taux de pauvreté sont les plus importants, et inversement).

\section{Pauvreté et inégalités}

La mesure de la pauvreté ne va pas sans discussion. Celle des inégalités est plus consensuelle dans la mesure où un outil fiable, le coefficient de Gini, permet des éclairages et des

23. Thimoty M. Smeeding, «Government Programs and Social Outcomes: Comparison of United States with Other Rich Nations», Alan J. Auerbach, David E. Card, John M. Quigley (dir.), Public Policy and the Income Distribution, Russel Sage Foundation, 2006. 
comparaisons précises. On sait que ce coefficient, entre o et 100, permet de «mesurer» l'inégalité dans la distribution des revenus, au sens où o signifie que nous avons une parfaite égalité (de revenu dans ce cas-ci) et 100 indique un niveau extrême d'inégalité: dans ce cas limite, une seule famille recevrait tous les revenus. Ce coefficient, appliqué au «revenu disponible des familles» - cela permet de tenir compte de l'effet de la fiscalité - , et se rapportant à plusieurs pays, permet de comparer l'ampleur de l'inégalité dans la distribution des revenus et de faire ressortir les écarts d'un pays à l'autre.

Dans l'ensemble, on note une accentuation de l'inégalité du revenu disponible des familles dans les décennies 1980 et 1990, mais cela souffre certaines exceptions. Dans quelques pays (Danemark, Suisse, Irlande et France), la variation est presque nulle et aux Pays-Bas, il y a un resserrement des inégalités; pour le reste, nous assistons plutôt à un approfondissement des inégalités.

Les pays scandinaves ${ }^{24}$ se situent au départ à un seuil très bas $(22,1)$, révélant par là une structure particulièrement égalitaire de la distribution du revenu disponible des familles. L'augmentation moyenne de 3 points laisse ce groupe de pays dans une situation enviable $(25,2)$, même s'il faut mettre en lumière deux considérations. D'abord, au sein de ce groupe, deux pays (Suède et Finlande) laissent pointer une progression assez nette de l'inégalité, mais, en même temps, il s'agit de pays, au départ, singulièrement égalitaires. Ensuite, cette progression relative, associée à une stabilité des pays regroupés sous le terme d'«économie sociale de marché (ÉSM)

24. Nous reviendrons ultérieurement sur la tripartition entre économie libérale de marché (ÉLM) et économie sociale de marché (ÉSM), scindée entre le modèle «nordique» et le modèle «continental», proposée par Jonas Pontusson. 
TABLEAU 1.2

Distribution du revenu disponible des familles, exprimée par le coefficient Gini, 1979-2000

\begin{tabular}{|l|c|c|}
\hline & La plus ancienne & La plus récente \\
\hline Économie sociale de marché nordique & $\mathbf{2 2 , 1}$ & $\mathbf{2 5 , 2}$ \\
\hline Danemark (1987-1997) & 25,4 & 25,7 \\
\hline Finlande (1987-2000) & 20,9 & 24,7 \\
\hline Norvège (1979-2000) & 22,3 & 25,1 \\
\hline Suède (1981-2000) & 19,7 & 25,2 \\
\hline Économie sociale de marché continentale & $\mathbf{2 5 , 3}$ & $\mathbf{2 6 , 7}$ \\
\hline Autriche (1987-1997) & 22,7 & 26,6 \\
\hline Belgique (1985-2000) & 22,7 & 27,7 \\
\hline Allemagne (1981-2000) & 24,4 & 26,4 \\
\hline Pays-Bas (1983-1999) & 26,0 & 24,8 \\
\hline Suisse (1982-1992) & 30,9 & 30,7 \\
\hline Économie libérale de marché & $\mathbf{2 9 , 3}$ & $\mathbf{3 3 , 0}$ \\
\hline Australie (1981-1994) & 28,1 & 31,1 \\
\hline Canada (1981-2000) & 28,4 & 30,2 \\
\hline Irlande (1987-2000) & 32,8 & 32,3 \\
\hline Royaume-Uni (1979-1999) & 27,0 & 34,5 \\
\hline États-Unis (1979-2000) & 30,1 & 36,8 \\
\hline France (1979-1994) & $\mathbf{2 9 , 3}$ & $\mathbf{2 8 , 8}$ \\
\hline Italie (1986-2000) & $\mathbf{3 0 , 6}$ & $\mathbf{3 3 , 3}$ \\
\hline
\end{tabular}

Source: Jonas Pontusson, Inequality and Prosperity: Social Europe vs. Liberal America, Cornell University Press, 2005 , p. 36 (notre traduction).

continentale», a pour effet combiné que ces deux groupes ont des coefficients similaires $(25,2$ contre 26,7$)$. Les pays anglo-saxons, dits d'«économie libérale de marché», creusent davantage cet écart avec 6,3 points entre les deux coefficients, marquant une «séquence néolibérale» (1980-2000). Dans ce groupe, c'est le Royaume-Uni et les États-Unis qui ont, et de loin, les coefficients les plus élevés $(34,5$ et 36,8$)$, ce qui est révélateur de fortes inégalités de revenu disponible des familles. Rappelons qu'en 1998 - et les choses se sont accentuées par après - le cinquième des familles américaines 
ayant le moins de revenus recevaient 4,2\% du revenu total, alors que le cinquième des familles les plus fortunées en drainaient 47,3\% ${ }^{25}$. C'est même au Royaume-Uni que l'inégalité s'accroît le plus sur cette séquence de deux décennies. On dégage ici des regroupements assez proches de ceux observés sur la question de la pauvreté.

Aussi, au regard des bons résultats en termes de croissance économique connus par la Grande-Bretagne et les États-Unis depuis le début des années 1980, la question de l'articulation entre création de richesse, inégalités et pauvreté se pose avec acuité.

D’un point de vue analytique, l'équation entre inégalités et pauvreté ne va pas de soi. L'inégalité et les écarts de revenus ne signifient pas qu'il y ait nécessairement un appauvrissement de la population. Il est possible de jouer avec certaines hypothèses qui soulignent que la relation entre ces deux termes n'est pas mécanique. Par exemple, la population la moins dotée peut connaître une amélioration de sa situation et, dans le même temps, les mieux dotés profiter d'une amélioration encore plus forte; la pauvreté aura baissé et les inégalités se seront creusées. À l'inverse, si la situation des moins dotés se détériore, mais que celle des mieux dotés connaît une détérioration encore plus importante, les inégalités auront décru, mais la pauvreté aura augmenté.

Au-delà de ces hypothèses, la vulgate libérale stipule qu'une certaine dose d'inégalité peut être bénéfique collectivement, puisqu'elle pousse chacun à produire davantage afin de maximiser ses revenus. Dans cette perspective, introduisant un glissement conceptuel entre égalité et équité, les inégalités seraient justes dans la mesure où elles récompensent l'effort

25. Gary Burtless, Timothy M. Smeeding, «The Level, Trend, and Composition of Poverty», Sheldon Danziger, Robert H. Haveman (dir.), Understanding Poverty, op. cit. 
et stigmatisent l'échec. Cependant, cette hypothèse méritocratique trouve peu d'appuis empiriques ${ }^{26}$. Même l'équation simpliste qui corrèle le revenu à l'activité économique est mise à mal par une donnée majeure de la configuration actuelle: la désarticulation des questions de revenu et de travail. Tandis qu'une partie non négligeable de l'explosion des plus hauts revenus ces dernières décennies ne provient pas du fruit d'un travail, mais de la détention de patrimoine (biens immobiliers et capitaux financiers), à l'autre extrémité du spectre social, on constate le développement d'une frange importante de travailleurs pauvres. Cette reconfiguration de la pauvreté pose de nouveaux enjeux analytiques.

\section{Travailler et être pauvre: nouveaux enjeux analytiques}

Le phénomène du travailleur pauvre est davantage connu et documenté en Amérique du Nord, mais devient de plus en plus présent pour les pays européens. Les États-Unis possèdent la proportion la plus élevée de travailleurs mal payés ${ }^{27}$ : en 2001 près de $5 \%$ des personnes travaillant au moins 27 heures par semaine sont pauvres ${ }^{28}$, et nous parlons ici du seuil de pauvreté officiel, soit environ $40 \%$ de la médiane du revenu disponible. Cela touche particulièrement les jeunes de 16 à 25 ans; cette pauvreté est davantage concentrée chez les minorités visibles, et plus importante chez les femmes que chez les hommes.

26. Jonas Pontusson, Inequality and Prosperity: Social Europe vs. Liberal America, Cornell University Press, 2005.

27. Timothy M. Smeeding, «Government Programs and Social Outcomes: Comparison of United States with Other Rich Nations», Alan J. Auerbach, David E. Card, John M. Quigley (dir.), Public Policy and the Income Distribution, op. cit.

28. Abraham T. Mosisa, «The working poor in 2001», Monthly Labour Review, vol. 126, $\mathrm{n}^{\text {os }} 11 \& 12,2 \mathrm{OO} 3$. 
Soulignons que la part des emplois faiblement rémunérés ne saurait être expliquée par la proportion de travailleurs peu qualifiés. Il n'y a pas de relation simple et linéaire et la situation est fortement contrastée d'un pays à l'autre ${ }^{29}$.

Une étude de l'OCDE ${ }^{30}$ fait ressortir une corrélation positive entre l'importance des faibles salaires et l'importance de l'inégalité des salaires (mesurée par le ratio 50/10, pour les déciles de revenu). En ce sens: la forte inégalité des salaires va de pair avec le taux élevé de bas salaires. Les bas salaires tendent à être liés à des travailleurs présentant des caractéristiques similaires d'un pays à l'autre: faible formation, jeunes travailleurs, femmes et employés dans la vente et les services de base. Les différences repérées sont davantage le fait d'une différenciation d'arrangements institutionnels, dont l'existence d'un salaire minimum et la négociation collective $^{31}$.

D'ailleurs, la fréquence de la pauvreté parmi les chômeurs varie considérablement selon les pays. Les données compilées indiquent que, dans chaque pays, ceux qui entrent en chômage ont une propension beaucoup plus forte à devenir pauvres $^{32}$. Mais des différences importantes entre les pays sautent aux yeux; la propension à devenir pauvre est beaucoup plus faible au Danemark et aux Pays-Bas, alors qu'elle est très élevée en Grande-Bretagne et en Belgique. L'incidence est généralement beaucoup plus forte pour les hommes que pour les femmes. Si le chômage est une source avérée de

29. Graeme Cooke, et Kayte Lawton, Working Out of Poverty. A study of the low-paid and the «working poor», Institute for Public Policy Research, 2008.

30. OCDE, Earnings Inequality, Low-paid Employment and Earning Mobility, Employment Outlook, 1996.

31. Graeme Cooke, Kayte Lawton, Working Out of Poverty. A study of the low-paid and the "working poor»,op. cit.

32. Duncan Gallie, Serge Paugam, Sheila Jacobs, «Unemployment, Poverty and Social Isolation. Is There a Vicious Circle of Social Exclusion?», European Societies, vol. 5, n ${ }^{\circ}$ 1, 2003. 
risque de pauvreté, cette relation est non univoque puisqu'elle dépend largement de la structure du marché de l'emploi, de l'éventail des dispositifs publics (formation, reclassement, aide au revenu, etc.). Mais la dynamique d'objectivation du statut du «pauvre» passe également par la pesanteur de représentations sociales qui peuvent instituer des cadrages différenciés: «l'assisté», «la victime», «le profiteur», etc. Cette articulation des dynamiques s'incarne différemment selon les pays et les périodes; l'intérêt de la démarche comparative est précisément d'éclairer les points de convergence et les spécificités irréductibles des cas que nous avons choisis.

\section{Comparer les fondements de la pauvreté}

Comprendre et mesurer «la pauvreté» exige de décloisonner le regard, afin de prendre en considération une multiplicité de facteurs qui font de celle-ci non seulement le produit d'une privation économique, mais un statut social, individuel et collectif, et le révélateur de compromis politiques au sein d'une société donnée. Aussi, afin de rendre compte de cette réalité complexe, nous procéderons par l'application systématique d'une grille de lecture à quatre cadrans, au sein de nos quatre cas (Grande-Bretagne, Danemark, Québec, France).

\section{Une grille d'analyse à quatre cadrans}

Le premier cadran concerne la structure du marché du travail. Au-delà des indicateurs économiques traditionnels (taux de chômage, taux d'activité, taux de croissance), nous porterons attention à la morphologie de ce marché, avec notamment le poids des emplois précaires, la fréquence des rotations, les types d'emplois créés, etc.

Le deuxième cadran renvoie à la structure des compromis sociaux, autrement dit au modèle de «concorde sociale». On 
désigne par là le poids respectif des partenaires sociaux, le plus ou moins grand dirigisme de l'État, la portée des accords socioéconomiques négociés.

Le troisième cadran synthétise l'éventail des politiques publiques à l'œuvre, et notamment des dispositifs d'activation. On mettra l'accent sur la dynamique de convergence et de reconduction de spécificités nationales dans l'application des outils a priori relativement standardisés du workfare.

Le quatrième cadran aborde le traitement politique de la pauvreté, à travers l'institutionnalisation d'une représentation du «pauvre». Comme le démontrent Paugam et Selz ${ }^{33}$, cette représentation est sujette à des évolutions structurelles et conjoncturelles. Parallèlement à des déclinaisons nationales nettement différenciées (entre stigmatisation et solidarité), on observe dans les périodes plus prospères une accentuation de cette stigmatisation, tandis que les séquences économiques plus difficiles sont propices à des jugements plus favorables, notamment quand se diffuse la perception selon laquelle chacun peut être touché par le chômage.

La conjugaison de ces quatre cadrans (graphique 1.1) permettra de formaliser une représentation de la configuration socioéconomique de chaque pays afin de mieux saisir in fine comment s'y insère la question de la pauvreté. Au-delà de l'exploration de chaque configuration, c'est la mise en dialogue entre des cas différents qui produira un certain nombre d'éclairages féconds.

33. Serge Paugam, Marion Selz, «La perception de la pauvreté en Europe depuis le milieu des années 1970. Analyse des variations structurelles et conjoncturelles. », Économie et statistique, $\mathrm{n}^{\mathrm{os}}$ 383-384-385, 2005, p. 283-305. 
GRAPHIQUE 1.1

Aux fondements de la pauvreté et des inégalités

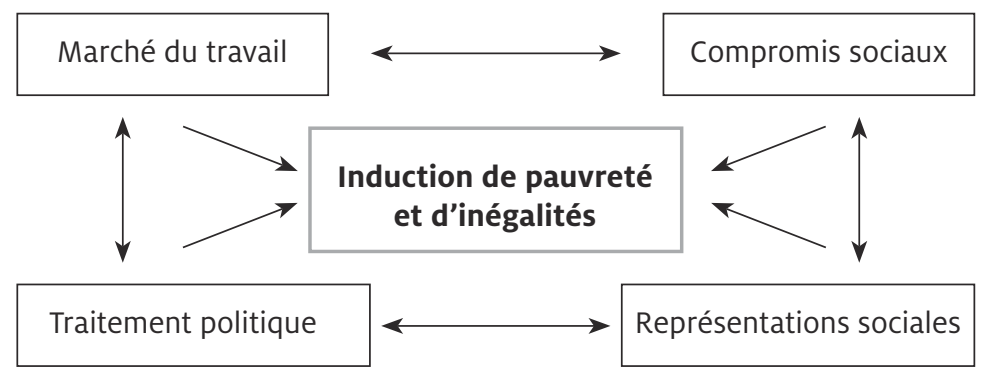

\section{Plan de l'ouvrage}

Le choix des cas étudiés s'est opéré autour de deux couples distincts: Grande-Bretagne et Danemark, Québec et France. Ainsi, nous mettons en relief l'opposition quasi idéale typique entre la situation de la Grande-Bretagne et du Danemark, si on reprend la dichotomie de Jonas Pontusson ${ }^{34}$ entre «économie libérale de marché» (ÉLM) et «économie sociale de marché» (ÉSM).

Cette schématisation est inspirée par un croisement entre les travaux des théoriciens de la variété des formes du capitalisme $^{35}$ et ceux déjà évoqués d'Esping-Andersen ${ }^{36}$. Les deux modèles se scindent selon trois critères: le degré et la modalité de coordination du milieu des affaires (coordination par le marché ou stratégique); le poids des syndicats et

34. Jonas Pontusson, Inequality and Prosperity: Social Europe vs. Liberal America, op. cit.

35. Peter J. Katzenstein, Small States in World Markets, Cornell University Press, 1985; Peter Hall, David Soskice (dir.), Varieties of Capitalism, Oxford University Press, 2001.

36. Gøsta Esping-Andersen, The Three Worlds of Welfare Capitalism, op. cit. 
des conventions collectives; et enfin la part des dépenses publiques dans le domaine social. Du côté des Économies libérales de marché, l'auteur regroupe les États-Unis, le RoyaumeUni, le Canada, l'Irlande, l'Australie et la Nouvelle-Zélande. Du côté des Économies sociales de marché, il distingue un sousgroupe «nordique» (Finlande, Danemark, Norvège et Suède), d'un second, «continental» (Allemagne, Autriche, Suisse, Belgique, Pays-Bas), selon les distinctions entre les modalités de protection sociale soulignées par Esping-Andersen (régime social-démocrate/régime corporatiste ou conservateur).

Quelques indicateurs permettent de mesurer l'opposition entre le Danemark et la Grande-Bretagne dans le domaine social, tandis qu'ils sont fréquemment présentés comme deux «modèles» (au sens normatif), dotés tous deux de fortes performances économiques et d'une grande flexibilité du marché du travail. Si on considère le «classement» des pays de l'OCDE en termes d'inégalité, selon le coefficient de Gini, au milieu des années 2000 (30 pays, coefficient moyen de o,31), on observe que le Danemark présente le coefficient le plus faible $(0,23)$, ce qui signifie la répartition des revenus la plus ouverte, tandis que le Royaume-Uni occupe la $23^{\mathrm{e}}$ place $(0,34)$, parmi les pays présentant les plus fortes inégalités ${ }^{37}$. Si l'on s'attache à la question de la pauvreté des enfants, on constate la même répartition, avec le Danemark en tête et le Royaume-Uni en queue de classement ${ }^{38}$.

37. Si les dernières places sont occupées par le Portugal, la Turquie et le Mexique, on peut souligner que les pays les plus représentatifs de l'économie libérale de marché ont des résultats très proches du Royaume-Uni puisqu'on trouve l'Irlande en $22^{\mathrm{e}}$ position, la Nouvelle-Zélande en $23^{\mathrm{e}}$ position (au même niveau que le Royaume-Uni) et les États-Unis en $27^{\mathrm{e}}$ position. Voir Croissance et inégalités: Distribution des revenus et pauvreté dans les pays de l'OCDE, octobre 2008.

38. Ibid. 
Une fois les deux polarités incarnées par le Danemark et la Grande-Bretagne posées, nous explorons les cas de la France et du Québec afin de nous interroger sur deux configurations «hybrides». Que ce soit dans le modèle d'EspingAndersen ou dans celui de Pontusson, la France cadre mal avec les typologies d'usage. On y observe des politiques sociales et des conventions collectives fortes, mais un dialogue social peu institutionnalisé et une syndicalisation faible. Le Québec, pour sa part, est fréquemment décrit comme une province en tension entre des politiques sociales volontaristes (notamment issues de sa Révolution tranquille des années 1960) et son appartenance à une configuration canadienne nettement libérale ${ }^{39}$. Pour les deux indicateurs précédemment indiqués (inégalités et pauvreté des enfants), la France et le Québec se classent d'ailleurs dans des positions médianes, à distance des deux polarités précitées.

Dans chaque configuration, on constate la production d'externalités positives, mais aussi négatives. La pauvreté de tel ou tel segment de la population n'est pas un «raté»du système, mais bien la conséquence «logique» du bouclage de chaque configuration. Les deux derniers chapitres de l'ouvrage opèrent un retour sur les dynamiques de convergence, d'emprunt de dispositifs et de brouillage des distinctions entre les configurations respectives. Nous posons la question - toujours périlleuse - du sens des transformations en cours dans l'action publique en matière de pauvreté.

39. Gérard Boismenu, Pascale Dufour, «Participation de l'État et de l'environnement social à l'aide aux personnes sans emploi», Recherches et Prévisions, $\mathrm{n}^{\circ}$ 91, mars 2008; Gerard W. Boychuk, Keith G. Banting, «Converging and Diverging Paradoxes: National and Sub-National Variation in Income Maintenance Programs in Canada and the United States», Richard G. Harris (dir.), North American Linkages: Opportunities and Challenges for Canada, University of Calgary Press, 2004; Alain Noël (dir.), Federalism and Labour Market Policy: Comparing Different Governance and Employment Strategies, McGill-Queen’s University Press, 2004. 
Sommes-nous dans un univers technique où les politiques et programmes mis en place récemment ne sont que des réponses par essai-erreur et apprentissage? Les configurations nationales ont-elles tendance à se rapprocher avec le passage du temps? Être une personne en situation de pauvreté à Copenhague, Londres, Paris ou Montréal, qu'est-ce que cela change? Comment les fondements différenciés de la pauvreté nous renseignent-ils sur les transformations des trajectoires de l'État social?

Nous explorons dans un premier temps une piste peu empruntée, celle de la construction des indicateurs qui guident nos lectures des modèles et des trajectoires des États sociaux. Cette mise en perspectives des «mesures» qui soustendent les comparaisons nous permet d'informer la question de la convergence ou de la divergence des modèles à partir d'un point de vue original. Et si notre alphabet académique (taux de pauvreté, produit national brut, taux de chômage, etc.) s'accompagnait d'une forme de «mise à plat» plutôt que d'une «mise en perspective» des fondements de la pauvreté dans nos sociétés? 



\section{CHAPITRE 2}

\section{Les arêtes du triangle danois}

Pays cité à tout va ${ }^{1}$, le Danemark est souvent posé comme le modèle de référence pour la restructuration de la protection sociale, car cette dernière, particulièrement développée, est couplée à une grande souplesse dans l'organisation des relations de travail et à des indicateurs sociaux plutôt impressionnants pour la redistribution et l'inclusion sociale. L'évolution du taux de chômage au Danemark témoigne d'une dégradation puis d'une amélioration spectaculaire: de l'ordre de $5 \%$ en 1975, il croît dans les années 1980 pour atteindre un pic à plus de $12 \%$ en 1993, avant de redescendre à $5 \%$ au début des années 2000 (moins de $3 \%$ au début 2008).

Le chômage des jeunes, qui suscite des inquiétudes dans de nombreux pays européens avec un taux autour de 18,5\% en 2005, est deux fois moins important au Danemark (8,6\% en 2005). Pour étayer cette image de «bon élève», soulignons qu'à partir de la seconde moitié des années 1990, le Danemark est devenu le pays de l'Union européenne ayant le taux d'emploi le plus élevé, le taux de pauvreté le plus bas et le niveau

1. Michel Hastings, «Dieu est-il nordique?», Revue internationale de politique comparée, vol. 13, n³ 2006, p. 377-390. 
GRAPHIQUE 2.1

Taux de chômage, pays européens et Danemark, 1970-2006

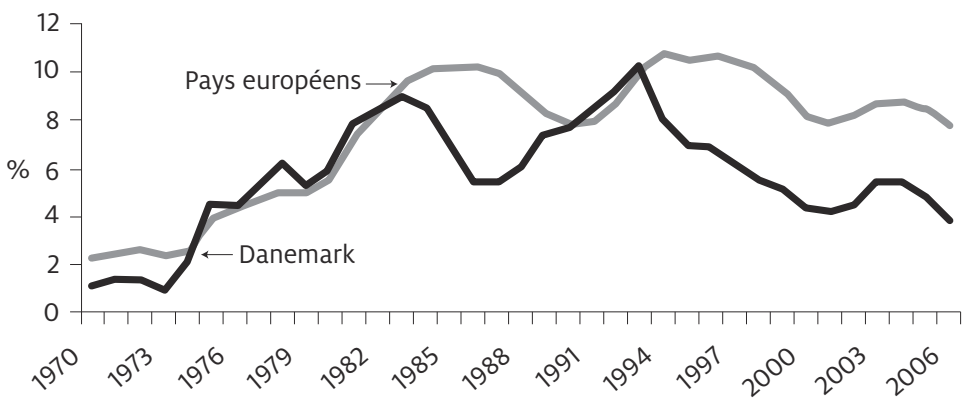

Source: Torben M. Andersen, Michael Svarer, Flexicurity - Labour Market Performance in Denmark, University of Aarhus, 2007, p. 2.

d'inégalité des revenus le plus bas ${ }^{2}$. La croissance de la production intérieure eu égard à la population est estimée à $3,6 \%$ en 2005 et à 2,7\% en 2006, taux qui sont supérieurs à la moyenne des pays de l'UE. Parallèlement, l'évolution positive du chômage depuis 1993 se boucle à des finances publiques saines (augmentation des surplus budgétaires, diminution de la dette publique) et ce, sans augmentation significative de l'inflation ${ }^{3}$.

Pour expliquer cette success-story, il est souvent fait référence à la construction d'un «triangle d'or» de la «flexicurité» danoise. On désigne ainsi l'articulation vertueuse de trois éléments: une flexibilité maximale du marché de l'em-

2. Jean-Claude Barbier (dir.), Analyse comparative de l'activation de la protection sociale en France, Grande-Bretagne, Allemagne et Danemark, dans le cadre des lignes directrices de la stratégie européenne pour l'emploi, DARES, Ministère du Travail, 2006.

3. Per Kongshøj Madsen, «The Danish model of "flexicurity": experiences and lessons», European Review of Labour and Research, vol. 10, n² 2, 2004. 
ploi (facilité des licenciements et embauches), une sécurité de revenu prévisible et généreuse qui atténue grandement les risques sociaux (importance des transferts sociaux et services publics assurant l'inclusion sociale) et une politique active du marché du travail (reconversion et formation des travailleurs) ${ }^{4}$. Après être revenus sur les différentes dimensions de cette configuration, nous explorerons les dynamiques d'inclusion et d'exclusion sur lesquelles elle s'appuie et qu'elle renforce.

\section{Un marché du travail flexible et dynamique, une population très active}

Le marché du travail danois est particulièrement dynamique et sa main-d'œuvre est la plus mobile d'Europe ${ }^{5}$. On estime qu'entre 25 et $35 \%$ de la main-d'œuvre y change d'employeur chaque année. Entre un quart et un tiers de la main-d'œuvre connaît d'ailleurs une période de chômage chaque année ${ }^{6}$. Par exemple, en 1995, on a évalué que 800 ooo personnes, soit près du tiers de la population active, ont été au chômage au cours de l'année. Mais $40 \%$ l'ont été moins de $20 \%$ de l'année tandis que $15 \%$ l'ont été pour plus de $80 \%$ de l'année. On remarque d'ailleurs qu'au cours des dernières années, le chômage de longue durée a décru, se stabilisant autour de

4. Katrine Søndergård, «La flexicurité danoise - et tout ce qui l'entoure», Chronique internationale de l'IRES, $\mathrm{n}^{\circ} 110,2008$, p. 44-45; Per Kongshøj Madsen, «How Can It Possibly Fly? The Paradoxe of a Dynamic Labour Market in a Scandinavian Welfare State», John L. Campbell, John A. Hall, Ove Kaj Pedersen (dir.), National Identity and the Varieties of Capitalism: The Danish Experience, McGill-Queen's University Press, 2006.

5. Per Kongshøj Madsen, «How Can It Possibly Fly? The Paradoxe of a Dynamic Labour Market in a Scandinavian Welfare State», op. cit.

6. Thomas Bredgaard, Flemming Larsen, «The Transitional Danish Labour Market: Understanding a Best Case, and Policy Proposals for Solving Some Paradoxes», CARMA, Aalborg University, Research Paper, 2006. 
20 \% du chômage total 7 . Dans une perspective comparée, l'importance respective du chômage de court et long terme place le Danemark dans une situation intermédiaire. En effet, au sein des pays d'économie libérale de marché, dont nous examinons dans le prochain chapitre le cas britannique, le chômage de court terme domine tandis que celui de long terme n'occupe qu'une portion congrue. À l'opposé, les pays d'économie sociale de marché connaissent un chômage de long terme souvent plus important et problématique, comme en Belgique ou en Allemagne. Dans une configuration différente, les pays d'Europe du Sud sont également aux prises avec un chômage de longue durée très important.

Par ailleurs, au Danemark, le taux d'emploi très élevé est relativement bien distribué: il atteint 75,9\% en 2005, avec un taux pour les femmes presque aussi élevé que pour les hommes, sauf pour les catégories d'âge de 50 ans. Notons d'ailleurs qu'au sein de la classe d'âge 55-64 ans, le taux d'emploi est de l'ordre de 50,5\%, alors qu'il n'est que de 42,5\% en moyenne pour l'Europe des $25^{8}$. Le vieillissement de la population représente à ce titre l'un des grands défis auxquels le Danemark est confronté. On estime en effet que sur 100 personnes actives, les personnes âgées passeront de 22,6 en 2005 à 40 en 2050 , ce qui signifie une croissance des dépenses afférentes de 4,8\% du PNB en 2050. La situation conduit à considérer sérieusement le report de l'âge de la retraite aussi

7. Helge Albrechtsen, «Do We Need the Unemployed as a Forth Industrial Relations Actor? - The case of the United Kingdom, the Federal Republic of Germany and the Denmark within Framework of the European Employment Strategy», Jens Lind, Herman Knudsen, Henning Jørgensen (dir.), Labour and Employment Regulation in Europe, Peter Lang, 2004.

8. Danemark, National Report on Strategies for Social Protection and Social Inclusion, Ministry of Social Affairs \& Ministry of Interior Affairs and Health, 2006; Per Kongshøj Madsen, «The Danish model of "flexicurity": experiences and lessons», European Review of Labour and Research, vol. 10, n" 2, 2004. 
bien pour ce qui est de la «retraite précoce» (de 60 à 62 ans), que pour l'âge normal de la retraite (de 65 à 67 ans).

Pour saisir la structure de ce marché du travail, avec une population très active et mobile, il faut cerner sa régulation par une politique d'activation.

\section{Une activation reposant sur le développement de l'employabilité des travailleurs}

L'activation danoise est particulière dans le sens où elle articule simultanément un niveau de protection de l'emploi très limité, une flexibilité dans les relations de travail et un processus de prise en charge collective des risques sociaux. Pour comprendre sa genèse, notons qu'au tournant des années 1990, le chômage élevé a été associé principalement à des facteurs structurels. L'introduction de politiques d'activation de la main-d'œuvre se voulait une réponse par laquelle il devenait possible de lier motivation des chômeurs à trouver et à accepter un emploi, et qualification de ceux qui n'étaient pas en mesure de se trouver un emploi par eux-mêmes ${ }^{9}$. Sans gommer complètement la part de la motivation et de l'incitation des travailleurs à s'insérer sur le marché du travail, la politique met l'accent sur l'offre de travail adaptée aux besoins des entreprises et sur l'augmentation de la qualification des individus. La question est d'autant plus importante que les problèmes sociaux sont alors perçus comme liés à l’intégration sur le marché du travail ${ }^{10}$. Pour ce qui est des

9. Christian Albrekt Larsen, «Policy paradigms and cross-national policy (mis)learning from the Danish employment miracle», Journal of European Public Policy, vol. 9, $\mathrm{n}^{\circ}$ 5, 2002; Thomas Bredgaard, Flemming Larsen, Per Kongshøj Madsen, The Flexible Danish Labour Market - A Review, Aalborg University, 2005.

10. Flemming Larsen, Mikkel Mailand, «Danish Activation Policy: The Role of the Normative Foundation, the Institutional Set-up and Other 
attitudes des personnes, le problème ne résiderait pas tant dans la générosité de la couverture sociale que dans le manque d'attachement au marché du travail. D'où l'élaboration d'un discours sur le contrat moral entre les citoyens et la société (droits et devoirs respectifs) et l'introduction de mesures plus conditionnelles et disciplinaires. Cela éloigne la situation danoise des préceptes conventionnels de l'universalisme à tout crin tout autant que de l'individualisme forcené et du «laisser-faire».

Ces préceptes s'incarnent dans un système d'emploi hybride. D'un côté, les employeurs peuvent ajuster leur besoin de main-d'œuvre par des mises à pied avec un court préavis, si bien que le niveau de flexibilité du marché du travail danois se compare à des marchés du travail libéraux ${ }^{11}$.

Mais de l'autre, avec le système de sécurité sociale et les programmes d'activation, le Danemark possède toutes les caractéristiques des États sociaux-démocrates. La spécificité de l'articulation entre la flexibilité et la sécurité est que le second terme est en quelque sorte un préalable au premier: comme le soulignait le premier ministre Rasmussen, le haut niveau de sécurité sociale est une précondition pour un marché du travail flexible.

Cette situation hybride se donne à voir via le principe de l'activation, dans une tension entre universalisme et conditionnalité de l'aide. Ainsi, tous les citoyens ont droit à des prestations de revenu et à des services sociaux. La protection sociale est un droit du citoyen, l'universalisme et l'inclusion sont les principes fondamentaux de l'État social danois. Mais la protection sociale est liée au marché du travail. Tous

Drivers», Amparo Serrano Pascual, Lars Magnusson (dir.), Reshaping Welfare States and Activation Regimes in Europe, Peter Lang, 2007.

11. Per Kongshøj Madsen, Danish Labour Market Institutions and Employment Outcomes, Federal Ministry of Economics and Technology, 2007. 
les bénéficiaires deviennent sujets à un critère d'employabilité et de disponibilité au travail. Pour ce faire, le système assure des filières de formation, de qualification et d'expérience de travail, tout en introduisant une marge de conditionnalité aux droits à la sécurité du revenu. Si les prestations reçues sont généreuses (en comparaison des standards internationaux), cette dialectique des droits et des devoirs met plutôt l'accent sur les seconds ${ }^{12}$ puisque l'individu se doit d'améliorer ses qualifications en participant aux différentes mesures d'activation.

Les mesures se déclinent en quatre grandes catégories: l'emploi subventionné dans le secteur privé, l'emploi temporaire dans le secteur public, la formation (spécifique ou plus générale) et enfin une gamme de programmes assez hétérogènes: assistance en recherche d'emploi, relevé de profils de compétences, construction d'estime de soi, etc. Cette politique d'activation, qui fait écho aux préoccupations gouvernementales et des entreprises, trouve sa motivation dans la volonté de diminuer le taux structurel de chômage, d'accorder toute son importance au «capital humain», d'augmenter l'investissement en capital physique et en R\&D, d'enrayer la menace d'une offre de travail insuffisante. On pourrait affirmer que le chômage a poussé les décideurs politiques dans cette direction, alors que c'est davantage l'éventualité d'une pénurie de main-d'œuvre qui a provoqué la participation des employeurs ${ }^{13}$.

12. Wim van Oorschot, Peter Abrahamson, «The Dutch and Danish Miracles Revisited: A Critical Discussion of Activation Policies in Two Small Welfare States», Social Policy \& Administration, vol. 37, nº 3, 2003.

13. Cathie Jo Martin, «Corporatism in the Post-Industrial Age: Employers and Social Policy in the Little Land of Denmark», John L. Campbell, John A. Hall, Ove Kaj Pedersen (dir.), National Identity and the Varieties of Capitalism: The Danish Experience, McGill-Queen's University Press, 2006. 
L'objectif de cette politique est d'aider tous les bénéficiaires à accéder à une forme d'insertion dans la société active, même s'il ne s'agit pas d'une intégration dans un emploi standard sur le marché du travail. Il n'est donc pas surprenant que près de $4 \%$ de la main-d'œuvre soit à un moment donné dans un quelconque programme d'activation, voire que près de $11 \%$ le soit pour les plus jeunes 16-24 ans ${ }^{14}$. Les programmes du marché du travail sont mis en œuvre par le système public d'emploi pour les assurés et par les gouvernements locaux pour les non-assurés. Les Labour Market Councils régionaux prennent en charge l'administration des politiques du marché du travail. Ces structures sont inclusives parce qu'elles ont l'obligation légale d'une représentation égale des syndicats, des autorités locales et des employeurs privés. Elles créent ainsi un espace de négociation entre les intérêts des organisations impliquées dans la politique d'emploi et expriment les géographies des marchés locaux du travail ${ }^{15}$. Ajoutons que le renforcement des partenariats sociaux sur le plan local assure un certain élément de responsabilité et de démocratie, qui va de pair avec le principe de construction de consensus et de l'économie négociée en tant que solidarité sociale. Il est d'ailleurs intéressant de noter que la réforme instaurant l'activation obligatoire a été essentiellement menée par les partenaires sociaux, avec peu d'intervention gouvernementale ${ }^{16}$ et qu'elle bénéficie d'un

14. Wim van Oorschot, Peter Abrahamson, «The Dutch and Danish Miracles Revisited: A Critical Discussion of Activation Policies in Two Small Welfare States», Social Policy \& Administration, vol. 37, $\mathrm{n}^{\circ}$ 3, 2003.

15. David Etherington, Martin Jones, «Beyond contradictions of the workfare state? Denmark, welfare-through-work, and the promises of jobrotation», Environment and Planning C: Government and Policy, vol. 22, $\mathrm{n}^{\circ}$, 2004, p. 133-143.

16. Cathie Jo Martin, «Corporatism in the Post-Industrial Age: Employers and Social Policy in the Little Land of Denmark», National Identity and the Varieties of Capitalism: The Danish Experience, op cit. 
bon appui politique au sein de la population en général, ainsi que des chômeurs à long terme ${ }^{17}$.

Comme nous allons le voir maintenant, le soubassement normatif de ces dispositifs socioéconomiques d'activation du marché de l'emploi se retrouve dans le traitement politique de la pauvreté.

\section{Le traitement politique de la pauvreté : I'universalisme et le primat de l'inclusion comme matrice sociale}

À l'évidence, le Danemark est une société qui trouve de grandes vertus à un certain égalitarisme dans la distribution sociale de la richesse. L'idée maîtresse est que tous les citoyens aient accès, indépendamment de leurs revenus, à des services de base de grande qualité (services médicaux, hospitalisation, éducation, garderies, soins aux personnes âgées et aux personnes handicapées). De même, il est considéré normal que la vaste majorité de la population active trouve un emploi ou soit en formation générale ou spécialisée. On le perçoit aisément, ces injonctions normatives s'articulent aux finalités économiques de l'activation, comme pour la formation continue ou encore la possibilité pour les ménages ayant des enfants de concilier vie familiale et vie professionnelle pour les deux parents, grâce aux services de garde par exemple.

Ces postulats d'inclusion sociale ne semblent pas être de vaines dispositions, car, si on retient la comparaison avec l’Union européenne des 25 en 2003, le taux de pauvreté (60\% de la médiane) est nettement inférieur (10 contre $16 \%$, soit environ $60 \%$ de moins) à celui des pays européens, et la

17. Anders Rosdahl, Hanne Weise, «When all must be active - workfare in Denmark», Ivar Lødemel, Heather Trickey (dir.), «An Offer You Can't Refuse»: Workfare in International Perspective, Policy Press, 2001. 
TABLEAU 2.1

Les indicateurs de la cohésion sociale pour l'Union européenne, en \%

\begin{tabular}{|l|c|c|c|c|c|c|c|}
\hline & A & B & C & D & E & F & G \\
\hline UE 15 & 15 & 9 & 4,4 & 9,8 & 9,6 & 3,0 & 18,1 \\
\hline Irlande & 21 & 13 & 4,5 & 10,8 & 8,5 & 1,3 & 12,1 \\
\hline Portugal & 20 & 15 & 6,5 & 5,1 & 5,3 & 1,8 & 41,1 \\
\hline Grèce & 20 & 14 & 5,7 & 4,5 & 9,0 & 5,1 & 15,3 \\
\hline Italie & 19 & 13 & 4,8 & 7,0 & 9,7 & 5,3 & 24,6 \\
\hline Espagne & 19 & 10 & 5,5 & 6,1 & 7,2 & 3,9 & 29,8 \\
\hline Royaume-Uni & 17 & 10 & 4,9 & 17,0 & 10,9 & 1,1 & 16,7 \\
\hline France & 15 & 9 & 4,0 & 9,3 & 10,4 & 2,8 & 13,3 \\
\hline Belgique & 13 & 7 & 4,0 & 13,8 & 14,2 & 3,5 & 12,4 \\
\hline Luxembourg & 12 & 9 & 3,8 & 2,8 & 6,3 & 0,8 & 17,0 \\
\hline Autriche & 12 & 7 & 3,5 & 4,4 & 7,5 & 0,8 & 9,5 \\
\hline Allemagne & 11 & 6 & 3,6 & 9,3 & 10,0 & 4,0 & 12,6 \\
\hline Finlande & 11 & 6 & 3,5 & n/a & n/a & 2,3 & 9,9 \\
\hline Danemark & 11 & 5 & 3,1 & n/a & n/a & 0,9 & 10,0 \\
\hline Pays-Bas & 11 & 5 & 3,8 & 7,2 & 8,1 & 0,7 & 15,0 \\
\hline Suède & 10 & n/a & 3,4 & n/a & n/a & 1,0 & 9,0 \\
\hline
\end{tabular}

A Les personnes ayant un revenu net inférieur à $60 \%$ de la médiane nationale (2001)

B Les personnes ayant un revenu net inférieur à $60 \%$ de la médiane nationale l'année en cours et les 2 ou 3 années précédentes (2001)

C Rapport par quintile des revenus disponibles (2001)

D Rapport de la population des 0-17 vivant dans des ménages où personne ne travaille (2003)

E Rapport de la population des 18-59 ans vivant dans des ménages où personne ne travaille (excluant les ménages d'étudiants de 18-24 ans) (2003)

F Chômeurs de plus d'un an (en \% de la population active)

G Proportion des 18-24 ans avec moins d'un niveau secondaire d'éducation qui ne sont pas étudiants à temps plein ou en formation (2003)

Source: John Hills, Inequality and the State, Oxford University Press, 2004, p. 59 (notre traduction). 
pauvreté touche beaucoup moins les enfants et les personnes âgées, toute comparaison faite. Toujours dans une perspective européenne, on l'a déjà mentionné, l'inégalité est beaucoup moins prononcée, quelle que soit la méthode de mesure retenue ${ }^{18}$.

Cette caractérisation globale s'inscrit dans la durée, au sens où cela fait partie des données structurelles de l'organisation socioéconomique de cette société. Cela n'empêche pas des variations conjoncturelles, ni n'impose une permanence aux mécanismes de redistribution de la richesse ni aux risques de joindre les rangs des personnes pauvres, mais on ne peut qu'observer une certaine stabilité dans les indices relevant de la distribution de la richesse dans la société et quant à la circulation sociale des personnes.

Larchitecture de ces dispositifs de régulation des inégalités repose sur l'intervention publique via l'instrument fiscal et des dépenses publiques. Ainsi, en 2000, le total des dépenses publiques représentait 54,3\% du PIB. En large partie, les dépenses en protection sociale sont financées par les impôts et non par les contributions sociales des employés et entreprises ${ }^{19}$. L'impôt sur le revenu est en moyenne plutôt élevé, tout comme l'imposition marginale sur les revenus ordinaires $^{20}$ : le taux d'imposition est supérieur à $50 \%{ }^{21}$. Une étude de l'OCDE démontre que, pour le milieu des années 2000, cette fiscalité et les transferts réduisent au Danemark l'inégalité des revenus d'environ $45 \%$, ce qui est la politique

18. Danemark, National Report on Strategies for Social Protection and Social Inclusion, op. cit.

19. Philippe Egger, Werner Sengenberger (dir.), Decent Work in Denmark: Employment, Social Efficiency and Economic Security, International Labour Office, 2003.

2O. Per Kongshøj Madsen, Danish Labour Market Institutions and Employment Outcomes, Bonn, Federal Ministry of Economics and Technology, 2007.

21. OCDE, Croissance et inégalités: distribution des revenus et pauvreté dans les pays de l'OCDE, octobre 2008. 
redistributive la plus forte (à côté de la Suède et la Belgique) parmi les 24 pays étudiés, où la baisse est en moyenne d'un tiers $^{22}$.

Les prestations sociales et les services sociaux ne sont généralement pas - ou seulement d'une manière limitée - dépendants des revenus précédents ou de l'attachement au marché du travail. Cette intervention a un impact avéré sur l'état de la pauvreté. Par exemple, selon une étude d'Eurostat basée sur les revenus de 1997, la proportion de la population danoise qui recevait un revenu de moins de $60 \%$ de la médiane nationale diminuait de 30 à $11 \%$, une fois prises en compte les prestations sociales autres que les pensions $^{23}$. La comparaison avec les États-Unis est frappante, dans la mesure où la réduction des inégalités par l'instrument fiscal et les transferts y est deux fois moins forte que dans le cas danois ${ }^{24}$.

Le modèle d'État social danois repose donc sur trois piliers: l'universalisme, l'égalité et la solidarité. L'universalisme fonde l'ensemble de la sécurité du revenu et des services publics, faisant en sorte que tout citoyen est porteur de droits qu'il fait valoir au besoin, indépendamment de sa relation au milieu familial. La structure de financement de cette protection sociale applique le principe de la solidarité, d'où une imposition fiscale très progressive. Le principe d'égalité rend caduque l'introduction de distinctions de catégories et de démarche ciblée ${ }^{25}$. Mais ce modèle trouve sa justification et sa pertinence dans sa complémentarité ins-

22. Ibid.

23. Philippe Egger, Werner Sengenberger (dir.), Decent Work in Denmark: Employment, Social Efficiency and Economic Security, op. cit.

24. OCDE, Croissance et inégalités: distribution des revenus et pauvreté dans les pays de l'OCDE, op. cit.

25. Lise Lotte Hansen, «From Flexicurity to FlexicArity? Gendered Perspectives on the Danish model», Journal of Social Sciences, vol. 3, n 2, 2007. 
titutionnelle avec les processus sociaux de «dialogue social» et la politique active du marché du travail. Nous allons donc voir maintenant comment la régulation du marché du travail, et plus largement l'interventionnisme étatique et les principes d'universalisme et de solidarité, s'articulent à une maîtrise des antagonismes sociaux spécifique.

\section{Régulation du marché du travail et maîtrise des antagonismes sociaux}

L'élaboration des compromis touchant les règles et le mode d'organisation du marché du travail est fondée sur un partenariat entre acteurs aux intérêts divergents, mais qui s'imposent à eux-mêmes la recherche de compromis plus ou moins stables. On peut parler d'un conflit d'intérêts maîtrisé, comme résultante et non comme point de départ de la mise en relation des grands acteurs collectifs. Ceux-ci se limitent pour l'essentiel à deux grandes organisations: DA (Dansk Arbejdsgiverforening), l'organisation patronale principale fondée en 1898, et LO (Landsorganisationen i Danmark), la Fédération danoise des syndicats, fondée en 1896 et très majoritaire. Il faut noter à ce titre que le taux de syndicalisation des salariés est de $80 \%$ en 2006, c'est-à-dire parmi les densités d'adhésion les plus élevées en Europe ${ }^{26}$. Les deux tiers des syndiqués sont affiliés à LO. Ce système bipartite sans intervention gouvernementale est d'un grand intérêt pour toutes les parties qui peuvent négocier des accords reflétant leurs besoins respectifs. Par intérêt commun de trouver des compromis, ils s'imposent un dialogue constructif et une relation

26. Dans les pays scandinaves, les chômeurs restent membres des syndicats. Corrélativement, les syndicats développent des initiatives importantes pour les réinsérer, notamment en participant aux politiques d'activation. 
fondée sur le consensus plus que sur le conflit ${ }^{27}$. Cela est rendu possible grâce à la confiance entre acteurs, une confiance basée sur le respect des contrats signés, l'application des règles et la participation négociée aux décisions de ressources humaines. Ainsi, à la différence de ce qu'on observera par exemple dans le cas français, il n'y a pas de perception de la «précarité de l'emploi» dans la société danoise. Ce qui domine, c'est bien plus une «confiance sociale» installée depuis longtemps, confiance qui trouve ses racines dans le compromis de septembre 1899 (Septemberforliget) entre partenaires sociaux, sous la forme d'un «consensus conflic$\operatorname{tuel}^{28} »$. Depuis ce compromis fondateur, les deux parties prennent en charge des questions qui sont traitées dans d'autres pays par l'État, comme la durée du temps de travail, la fixation du montant du salaire minimum, ou encore la mise en place d'une juridiction prud'homale privée. Ce dialogue social contribue à perpétuer cette confiance et il devient très difficile pour un gouvernement ou quelque acteur de changer radicalement les postulats qui fondent le compromis social sans reconstruire le consensus. Cela n'exclut cependant pas l'innovation.

Quelques exemples permettent d'illustrer ce point cardinal de la construction du projet de société. Les partenaires sociaux ont émis, en 1987, une déclaration conjointe indiquant qu'ils prendraient en considération la performance macroéconomique et négocieraient les augmentations de salaire en deçà du niveau international de la croissance des salaires afin d'assurer la compétitivité de l'industrie danoise. Cette déclaration est maintenant à la base des négociations

27. Katrine Søndergård, «La flexicurité danoise - et tout ce qui l'entoure», op. cit.

28. Jean-Claude Barbier, «Apprendre vraiment du Danemark: réflexion sur le "miracle danois"», Centre d'études de l'emploi, vol. 18, n² 2, 2005, p. 3. 
de salaire au Danemark ${ }^{29}$. Des inflexions politiques sont également à l'œuvre, comme dans la réforme du marché du travail de 1993. À ce titre, le changement de discours sur les politiques d'activation a marqué la volonté de définir un instrument important pour lutter contre le chômage structurel. Parallèlement, le gouvernement social-démocrate cherchait à démontrer sa capacité à briser la courbe du chômage. Les réformes n'ont été possibles que grâce à un large appui public. Mais si la sphère politique a été motrice durant cette séquence, il faut souligner qu'au moment des réformes de 1999, les partenaires sociaux ont dominé le processus puisque la plupart des détails ont été déterminés par les négociations entre DA et $\mathrm{LO}^{30}$. Dernier exemple: en septembre 2003, la tentative du gouvernement conservateur-libéral de diminuer les dépenses des prestations d'assurancechômage a fait face à un front uni d'opposition des syndicats et des organisations d'employeurs qui l'ont bloquée ${ }^{31}$.

La régulation des relations industrielles concourt également à la construction des consensus puisqu'elles se caractérisent par un haut niveau de syndicalisation, un processus de décision décentralisé, un rôle de l'État limité à celui de facilitateur et la fréquence de consultations intensives,

29. Torben M. Andersen, Michael Svarer, Flexicurity - Labour Market Performance in Denmark, University of Aarhus, 2007.

30. Cathie Jo Martin, «Corporatism in the Post-Industrial Age: Employers and Social Policy in the Little Land of Denmark», National Identity and the Varieties of Capitalism: The Danish Experience, op. cit.; Henning Jorgensen, «Le rôle des syndicats dans les réformes sociales en Scandinavie dans les années quatre-vingt-dix», Revue française des affaires sociales, $\mathrm{n}^{\circ}$ 4, 2003, p. 121-150.

31. Per Kongshøj Madsen, "The Danish model of "flexicurity": experiences and lessons», European Review of Labour and Research, vol. 10, n 2, 2004; Per Kongshøj Madsen, «How Can It Possibly Fly? The Paradoxe of a Dynamic Labour Market in a Scandinavian Welfare State», National Identity and the Varieties of Capitalism: The Danish Experience, op. cit. 
formelles et informelles ${ }^{32}$. Si les négociations collectives sont plutôt décentralisées, les accords sont, pour leur part, extensifs et portent sur une multitude de thèmes, tout en assurant une flexibilité et une créativité. L'obligation de paix sociale durant la convention, généralement de 2 ou 3 ans, assure une certaine stabilité. Le taux très élevé de couverture des accords collectifs (85\% en moyenne, soit $100 \%$ dans le secteur public et $77 \%$ dans le secteur privé) établit un cadre général, tout en étant décliné localement pour assurer une grande adaptabilité ${ }^{33}$. La négociation des salaires suit une démarche de «décentralisation centralisée»: les organisations membres de LO et de DA négocient les salaires minimums au niveau sectoriel et, ce faisant, posent les lignes directrices pour la négociation salariale annuelle au niveau local. La standardisation de l'ajustement des salaires est favorisée par la considération du coût de la main-d'œuvre et du niveau des prix au Danemark et à l'étranger, tel qu'il est établi par le comité tripartite de statistique. Plus récemment, on a aussi substitué aux accords de régulation «fermés» des accords-cadres plus ouverts destinés à être complétés par des négociations locales d'entreprises pour qu'elles puissent les adapter.

L'évolution va vers des accords toujours plus larges, incluant des thèmes aussi variés que les régimes de pension, les jours de congés payés, les jours payés consacrés aux soins familiaux, le fonds de formation continue, le fonds de congé de maternité, etc. En incorporant des questions sociales dans les conventions collectives, il est possible d'établir des com-

32. Philippe Egger, Werner Sengenberger (dir.), Decent Work in Denmark: Employment, Social Efficiency and Economic Security, op. cit.

33. Katrine Søndergård, «La flexicurité danoise - et tout ce qui l'entoure», op. cit. 
promis dans une perspective plus large, ce qui contribue à maintenir un équilibre entre flexibilité et sécurité.

La tradition de la réglementation corporatiste du marché du travail danois a pour origine le compromis de septembre 1899 par lequel les syndicats ont accordé aux employeurs le droit de «disposer des travailleurs» en retour du droit des employés de s'organiser en syndicat. Cet événement a aussi mené à la tradition de laisser aux acteurs du marché du travail le soin de négocier les salaires et les conditions de travail sans l'intervention de l'État; il en est résulté l'établissement d'un marché du travail volontariste. Les principes reconnus du système de négociations collectives sont: le droit de s'organiser, le droit de l'employeur de gérer et contrôler le travail, le droit de mener des actions et l'obligation de paix sociale qui proscrit l'utilisation de la grève pendant une convention collective. Les politiques du marché du travail sont le résultat du processus de négociations collectives. Elles s'inscrivent dans la loi d'une manière limitée, mais tout de même soumise à un système spécifique d'arbitrage/tribunal industriel. En somme, les acteurs sociaux sont responsables de la majeure partie des normes. C'est ainsi que l'on peut dire que le Danemark combine intimement les régimes de relations industrielles néocorporatiste et pluraliste.

\section{Les revers de l'ambition universaliste}

Conçu dans une perspective universelle, le jumelage danois de la flexibilité et de la sécurité ne cible pas des groupes désavantagés par le marché du travail ou hors de lui. On ne peut cependant ignorer la différenciation des effets selon les groupes du marché du travail. Ainsi, la flexibilité ne concerne pas toutes les catégories de travailleurs: les salariés peu ou pas qualifiés changent davantage d'emploi durant l'année 
(30\% de ces salariés le font), alors que le phénomène est beaucoup plus limité pour ceux qui occupent des positions supérieures (16\% des senior managers ${ }^{34}$ ). La combinaison d'un marché du travail flexible et d'un large filet de protection social tend à avantager les travailleurs hautement qualifiés, mobiles et flexibles, capables de changer d'emploi et de fonction rapidement. De leur côté, les travailleurs non qualifiés sont plus souvent exposés à des «tests de productivité» par leur employeur et sont davantage exposés à la compétitivité internationale en raison du type d'emploi qu'ils ont tendance à occuper; ils sont donc davantage susceptibles d'être exclus du marché du travail.

Une part de la population potentiellement active est donc progressivement exclue du marché de l'emploi. Elle devient prestataire de transferts (en 20O2, le nombre de personnes recevant des prestations sociales était de 800 ooo, soit $16 \%$ de la population totale), mais également cible des différents processus d'activation que nous avons évoqués. C'est au travers du double traitement de cette catégorie, statistique et sociale, que se jouent en partie l'endroit et l'envers du «miracle danois». Au lieu d'être étiquetées «chômeurs», ces personnes exclues du marché de l'emploi sont «en congé» ou «en activation». Pour Abrahamson, si on fusionne ces données, on peut conclure que le taux des personnes en âge de travailler n'occupant pas un emploi ordinaire est, en 20O2, de 9,2\%, soit un taux $50 \%$ supérieur à celui du chômage enregistré $^{35}$. Pour les tenants du modèle danois, ce procédé va au-delà d'un jeu de reclassification et d'étiquetage: ces statuts ne sont pas équivalents au statut de chômeur, eu

34. Per Kongshøj Madsen, Danish Labour Market Institutions and Employment Outcomes, op. cit.

35. Peter Abrahamson, «Welfare Reform: Renewal or Deviation?», National Identity and the Varieties of Capitalism: The Danish Experience, op. cit. 
égard au rapport au marché du travail, à la probabilité de réinsertion professionnelle et d'inclusion sociale. Pour des observateurs davantage critiques, ces mesures d'activation n'éliminent pas le problème de ceux qui circulent d'un programme à l'autre, d'un statut d'assisté à celui de travailleur précaire. Nous aurions là des filières de relégation de la «main-d'œuvre inutile» et un processus de gestion et de circulation d'une population de laissés-pour-compte et non d'autonomie ${ }^{36}$.

En complément de ce problème transversal, doit être mise en relief la situation critique de la population immigrante. Le solide bilan danois concernant l'exclusion sociale occulte l'écart entre les immigrants et les Danois sur les principaux indicateurs, écart qui est plus important que dans les autres pays ${ }^{37}$. De façon générale, les immigrants et leurs descendants ont environ un taux de chômage deux fois plus élevé que la population moyenne ${ }^{38}$. Et, en particulier, la situation des immigrants originaires de pays non occidentaux représente un problème majeur pour le marché du travail danois. De 1980 à 2005 , le nombre d'immigrants et de leurs descendants croît de façon importante: leur représentation passe de 3 à 8,4\%. On calcule que 70,9\% de ces immigrants proviennent de pays non occidentaux ${ }^{39}$. En 2005, le

36. Wim van Oorschot, Peter Abrahamson, «The Dutch and Danish Miracles Revisited: A Critical Discussion of Activation Policies in Two Small Welfare States», Social Policy \& Administration, vol. 37, nº 3, 2003.

37. Panos Tsakloglou, Fotis Papadopoulos, «Poverty, material deprivation and multi-dimensional disadvantage during four life stages: evidence from the ECHP», Matt Barnes et al. (dir.), Poverty and Social Exclusion in Europe, Edward Elgar Pub, 2002.

38. Peter Abrahamson, «Welfare Reform: Renewal or Deviation?», op. cit.

39. John Andersen, Jørgen Elm Larsen et Iver Hornemenn Moeller, «The exclusion and marginalisation of immigrants in the Danish welfare society: Dilemmas and challenges», International Journal of Sociology and Social Policy, vol. 29, $\mathrm{n}^{\text {os }}$ 5/6, 2009, p. 274-286. 
taux d'emploi des immigrants de pays non occidentaux est de $46,1 \%$ contre $60,2 \%$ pour les immigrants venant de pays occidentaux et $74,6 \%$ pour les Danois. Si on fait entrer la variable homme-femme, on constate que les immigrantes venant de pays non occidentaux ont un taux d'emploi de $39,4 \%$ contre $71,5 \%$ pour les Danoises ${ }^{40}$. Ces immigrants connaissent donc un taux de chômage sans commune mesure, qui est particulièrement élevé pour la première génération, mais qui dure, toutes proportions gardées, pour les immigrants de la deuxième génération. Cela peut s'expliquer par certaines barrières, telles que le niveau inférieur d'éducation, la langue, la discrimination ${ }^{41}$. Des études ont montré que le chômage chez les immigrants non européens était fonction du niveau de scolarisation, de leur formation professionnelle dans le système danois et du nombre d'années de scolarité dans leur pays d'origine. On peut penser que c'est une question de simple adéquation professionnelle avec les besoins du marché du travail, mais on ne peut pas négliger l'effet de la socialisation au sein de la société danoise et de l'assimilation de l'éthique du travail qui parcourt les institutions danoises ${ }^{42}$. S'ajoute à cette question l'état de santé de ces populations qui, là encore, confirme leurs «handicaps sociaux». En somme, on constate une combinaison de déficience de scolarité/formation et de diminution de la demande du travail peu qualifié ${ }^{43}$. Mais rien ne dit que cela

40. Catharina Juul Kristensen, «Toward Two-tier Universalism? A Discussion of the Welfare Principle», Journal of Social Sciences, vol. 3, n 2, 2007.

41. Per Kongshøj Madsen, Danish Labour Market Institutions and Employment Outcomes, op. cit.

42. John Andersen, Jørgen Elm Larsen et Iver Hornemenn Moeller, «The exclusion and marginalisation of immigrants in the Danish welfare society: Dilemmas and challenges», op. cit.

43. Helge Albrechtsen, «Do We Need the Unemployed as a Forth Industrial Relations Actor?», op. cit. 
épuise la question comme le suggère la montée en force du parti d'extrême droite danois (Dansk Folkeparti), attisant la stigmatisation des immigrants, notamment de confession musulmane, auxquels est opposée la «fermeture ethnique». Fort de ces succès électoraux, ce parti est devenu dans les années 2000 la troisième force du parlement et est en poste au sein de la coalition gouvernementale depuis 2001. Parallèlement, les puissantes organisations syndicales ont longtemps entretenu une attitude relativement frileuse à l'égard des travailleurs immigrés ${ }^{44}$.

Alors que le vieillissement de la population danoise pourrait conduire à l'intensification du recours à la main-d'œuvre étrangère, on mesure l'ampleur des défis lancés aux valeurs d'universalisme et d'inclusion sociale de ce pays relativement homogène de 5 millions d'habitants.

44. Henning Jorgensen, «Le rôle des syndicats dans les réformes sociales en Scandinavie dans les années quatre-vingt-dix», op. cit. 

CHAPITRE 3

\section{La légitimité des inégalités et la question sociale en Grande-Bretagne}

À l'image du Danemark, la Grande-Bretagne présente un cas qu'on pourrait dire idéal-typique, dans le sens où elle concentre et accentue un certain nombre de traits qu'on retrouve de manière plus nuancée dans d'autres configurations nationales. Le Danemark présente une certaine exemplarité lorsqu'il s'agit d'évoquer le cercle vertueux de la flexicurité; s'il en est de même pour le modèle britannique, cette exemplarité est bien différente. Laccent mis sur la flexibilité en Grande-Bretagne trouve son point d'ancrage dans les vertus du marché, tandis que les formes publiques d'intervention sont subordonnées à une complémentarité définie d'abord en intériorisant les impératifs des acteurs privés. Dit autrement, les rigidités institutionnelles associées à l'intervention publique seront combattues, alors que la démarche disciplinaire, voire punitive, dans l'intervention sociale auprès des populations sera valorisée. C'est ce bouclage, bien différent du cas danois précédemment étudié, que nous allons mettre en lumière.

La situation britannique ne semble pas manquer d'atouts. La croissance de la richesse, eu égard à la population, est 
parmi l'une des plus élevées au cours des vingt dernières années du dernier siècle (1980-2000), en comparaison des pays européens et nord-américains. Le taux de chômage est assez faible, notamment pour le chômage de longue durée. Mais, d'un autre côté, la Grande-Bretagne reste fortement inégalitaire et la richesse par habitant en 2002 est l'une des moins élevées parmi les 18 pays comparables. Un coup d'œil rapide sur quelques indicateurs sociaux permet de dresser un bilan assez sombre sur le plan de l'inégalité et de la pauvreté. La pauvreté (définie à moins de $60 \%$ de la médiane du revenu net) est très élevée en termes relatifs, ce qui associe d'assez près ce pays aux pays d'Europe du Sud. C'est aussi une pauvreté qui tend assez fortement à s'inscrire dans la durée (en termes de persistance).

On comprend que la pauvreté et les inégalités semblent liées au modèle de développement socioéconomique et à l'organisation des rapports socioprofessionnels de la configuration britannique; en somme, ce serait le prix à payer de ce modèle libéral de relations de travail.

\section{Un marché du travail actif après de grandes difficultés}

À l'image du Danemark (mais selon un ordre séquentiel différent), la situation du marché du travail en GrandeBretagne affiche dans les années 2000 des signes positifs, après avoir connu des phases de fort chômage. Le marché du travail connaît de très grandes difficultés au cours des années Thatcher, puis, après une accalmie, éprouve de nouveau une exacerbation de la crise de l'emploi au début des années 1990. Avant l'arrivée du gouvernement Blair (1997), la situation se redresse, ce qui est confirmé par la suite, si bien que, depuis 1993, le taux de chômage fléchit constamment pour se situer à près de $5 \%$ dans les années 2000. En 2006, il 
atteint son niveau le plus bas depuis vingt ans et oscille entre $4,5 \%$ et $4,8 \%{ }^{1}$. Cette évolution est remarquable. Il est vrai qu'une tendance à la diminution du chômage s'enclenche dans la plupart des pays de l'OCDE au début des années 1990, mais cette tendance est plus précoce en GrandeBretagne, la diminution y suit un rythme plus rapide et la trajectoire ne se dément pas. D'ailleurs, si la situation du marché de l'emploi stagne ou se détériore dans la plupart des pays au début des années 2000 , le Royaume-Uni confirme la contraction du chômage ${ }^{2}$.

Le chômage de longue durée (12 mois et plus) a beaucoup diminué et se compare très avantageusement aux autres pays européens: alors qu'il est de 3,3\% pour l'Union européenne (des 15, en 2002), il n'est plus qu'à 1,1\% au Royaume-Uni. Ajoutons que le chômage de longue durée a diminué de plus de $75 \%$ entre 1997 et $2003^{3}$. Un regard panoramique permet de souligner qu'avant le gouvernement Blair, la part des travailleurs de 25 à 49 ans, qui étaient en situation de chômage de longue durée, variait peu. Les hommes étaient nettement plus touchés que les femmes. Depuis l'introduction du New Deal 25 plus par le gouvernement Blair, il y a un très net mouvement à la baisse et l'écart entre les hommes et les femmes sur ce registre s'est resserré très sérieusement. Dans l'ensemble, le chômage est surtout concentré sur la strate d'âge des jeunes, soit de 18 à 24 ans (ici on fait abstraction des 16 et 17 ans): l'écart est particulièrement important avec les 25 ans et plus, et il s'approfondit. En somme, le taux de chômage des

1. Anne Daguerre, «Les politiques de retour à l'emploi aux États-Unis, en Grande-Bretagne et en France», Critique internationale, nº 31, 2006.

2. Nicolas Grivel, Nathalie Georges, Dominique Méda, Les prestations et services d'accompagnement des demandeurs d'emploi: comparaisons internationales Suède, Pays-Bas, Royaume-Uni, Centre d'étude de l'emploi, 2007.

3. John Hills, Inequality and the State, Oxford University Press, 2004. 
jeunes a diminué de $25 \%$ au cours de la dernière décennie, mais ce taux reste trois fois celui du taux de chômage des travailleurs plus âgés.

Si l'on prend un peu de recul, on constate que l'évolution du chômage distingue le Royaume-Uni de plusieurs pays. Mis à part les États-Unis, les différents pays ont connu des années 1990 particulièrement préoccupantes sur le plan du chômage, leur situation se dégradant nettement par rapport à la situation britannique .

Pour caractériser le dynamisme du marché de l'emploi britannique, on peut le replacer dans le cadre européen. On observe alors que le taux d'emploi du Royaume-Uni est le quatrième plus élevé dans l'Union européenne des 25, dépassant d'ores et déjà la cible identifiée pour 2010 par la Stratégie de Lisbonne. Parallèlement, ce pays se situe également audelà pour deux autres objectifs : avec $65,9 \%$ de taux d'emploi pour les femmes, il dépasse l'objectif de $60 \%$, et avec 56,9\% pour les travailleurs âgés, il se situe clairement au-dessus des $50 \%$ visés 5 . Ce fort taux s'accompagne d'une augmentation caractérisée pour les femmes seules chefs de famille (on passe de 44 à $54 \%$ de 1996 à 2002). D'autres données mettent en relief que le taux d'emploi du parent seul a augmenté de 11,3 points de 1997 à 2006, pour atteindre 56,6\%. Cette évolution semble liée aux politiques gouvernementales mises en œuvre, avec le New Deal Lone Parent et le crédit fiscal ${ }^{6}$.

Il nous faut également souligner un autre trait distinctif du marché du travail britannique: une flexibilité se conjuguant, contrairement à ce que nous avons vu au Danemark,

4. Stewart Kitty, «Changes in poverty and inequality in the UK in international context», John Hills, Kitty Stewart (dir.), A More Equal Society? New Labour, Poverty, Inequality and Exclusion, Policy Press, 2005.

5. Royaume-Uni, UK National Report on Strategies for Social Protection and Social Inclusion 2006-2008, Ministry of Labour \& Social Affairs, 2006.

6. Ibid. 
avec une très grande précarité. En effet, la structure du marché du travail repose sur un fort turnover. Le chômage est transitoire et très important. Plusieurs travailleurs actifs repassent chaque année par le marché du travail, mais il est vrai que la majorité retrouve rapidement un emploi compte tenu du nombre élevé d'emplois vacants et des caractéristiques très disparates de ces emplois ${ }^{7}$. Le peu de contraintes associées au contrat de travail standard explique d'ailleurs le faible recours aux contrats de travail à durée déterminée, l'avantage relatif de ce dernier y étant moins manifeste. Par exemple, depuis 1985, certains droits liés à la sécurité d'emploi ne sont reconnus qu'après deux ans de travail continu; cette période fut ramenée à un an en 1999, mais elle n'était que de six mois avant $1985^{8}$.

Le travail à temps partiel y est par contre très répandu particulièrement chez les femmes, ce qui contribue à leur insécurité et à leur insuffisance de protection -, si bien, qu'avec l'Espagne et le Danemark, la Grande-Bretagne détient un taux du travail à temps partiel parmi les plus élevés en Europe 9 . La croissance des emplois à temps partiel ou temporaires signifie dans les faits que 30\% des employés n'ont plus de travail à plein temps ou à revenu stable. Parallèlement, les travailleurs sont de plus en plus touchés par des changements dans les heures de travail et par l'augmentation du nombre d'heures supplémentaires non rémunérées. Nombreux sont

7. Guillaume Delautre, Nathalie Georges, «De l'utilité d'un outil de profilage des demandes d'emploi: comparaison de trois expériences européennes", L'Observatoire de l'ANPE: l'essentiel, n 10, 2007.

8. Alex de Ruyter, John Burgess, «Growing Labour Insecurity in Australia and the UK in the Midst of Job Growth: Beware the Anglo-Saxon Model», European Journal of Industrial Relations, vol. 9, $\mathrm{n}^{\circ}$ 2, 2003.

9. Jochen Clasen, Daniel Clegg, «Unemployment Protection and Labour Market Reform in France and Great Britain in the 1990s: Solidarity Versus Activation?», Journal of Social Policy, vol. 32, n 3, 2003. 
ceux qui travaillent bien au-delà de la durée normale de la semaine de travail ${ }^{10}$.

Dans l'ensemble, l'encadrement du marché du travail au Royaume-Uni laisse d'ailleurs place à une grande flexibilité sur plusieurs rapports. Si l'on considère la législation sur la protection de l'emploi (LPE) - soit l'ensemble des règles qui régissent l'embauche et le licenciement des salariés, qui ont généralement pour objet de renforcer la sécurité de l'emploi pour les travailleurs - , force est de constater que le RoyaumeUni présente un profil particulièrement peu contraignant. Par exemple, on postule qu'une législation trop restrictive est sujette à freiner les décisions d'embauche, car elle suppose des coûts d'ajustement des effectifs pour l'employeur et des obstacles juridiques et institutionnels à l'embauche ${ }^{11}$.

En somme, on perçoit nettement une modalité d'articulation de la flexibilité et de la sécurité très différente du modèle danois. C'est également la représentation de la précarité qui différencie les deux configurations. Dans le modèle britannique, une architecture morale bien spécifique sous-tend les dispositifs de sécurité sociale et d'aide aux chômeurs.

\section{La stigmatisation de la pauvreté et la responsabilisation des individus}

Durant le règne des conservateurs, sous la férule notamment de Margaret Thatcher (1979-1990), on assiste à l'hégémonie d'une conception minimaliste de la protection sociale, réformée en 1988, et à la volonté arrêtée de réduire le rôle de l'État.

10. Alex de Ruyter, John Burgess, «Growing Labour Insecurity in Australia and the UK in the Midst of Job Growth: Beware the Anglo-Saxon Model», op. cit.

11. OCDE, Perspectives de l'emploi de l'OCDE. Stimuler l'emploi et les revenus, Paris, Organisation de coopération et de développement économiques, 2006. 
Fondamentalement, une crainte permanente inspire les politiques: en aidant ceux qui n'en auraient pas besoin, on crée un problème de dépendance et de pauvreté plus qu'on ne le résout. La réforme de l'assurance-chômage menée par John Major en 1996 va dans ce sens, en réduisant la durée des prestations, durcissant les conditions d'admissibilité et les contrôles (réforme du Job Seeker Allowance). Cette conception nourrit encore aujourd'hui la volonté d'activation des personnes vivant de prestations et motive l'obsession de tri à l'origine des réformes successives du système de protection sociale ${ }^{12}$.

Après dix-huit années de majorité gouvernementale conservatrice, la proportion de la population ne touchant que de faibles revenus a plus que triplé (de $8 \%$ à $25 \%$ ). Mais l'élection du gouvernement travailliste de Tony Blair en 1997 ne suscite pas une grande bifurcation, même si elle introduit davantage de dispositifs d'incitation, à côté de ceux plus contraignants. Pendant deux ans, les pouvoirs publics ont poursuivi la politique budgétaire des conservateurs, et aucune réduction des inégalités de revenus n'a été enregistrée ${ }^{13}$. Ce gouvernement a tablé sur la notion d'exclusion sociale pour s'engager dans des politiques centrées sur un plus large éventail d'objectifs outre la simple compensation de revenu ${ }^{14}$. Qui plus est, la lutte à l'inégalité économique, en particulier pour les plus hauts revenus, n'est pas à l'ordre du jour: l'égalité économique cède la place à l'égalité des chances, véritable

12. Hélène Zajdela, Ai-Thu Dang, Fondements normatifs des politiques d'activation: un éclairage à partir des théories de la justice, Centre d'étude de l'emploi, 2007.

13. Trine P. Larsen, Anne Daguerre, Peter Taylor-Gooby, Woman and the Labour Market - The National Childcare Strategy including Sure Start and the Integrated Child Credit, University of Kent, 2001.

14. Matt Barnes, Social Exclusion in Great Britain: An Empirical Investigation and Comparison with the EU, Ashgate, 2005. 
matrice de la «troisième voie» théorisée notamment par Anthony Giddens ${ }^{15}$.

Plus généralement, on peut observer sur le moyen terme, au-delà de la rupture présumée, une certaine continuité dans les soubassements normatifs de la politique d'activation entre les mandats conservateurs de Margaret Thatcher puis John Major, et la séquence travailliste du New Labour de Tony Blair puis Gordon Brown. Le leitmotiv pourrait se résumer à employment opportunity for all (opportunité d'emploi pour tous) complété par work for those who can, security for those who cannot (travail pour ceux qui le peuvent, sécurité pour ceux qui ne le peuvent pas). S’il y a incapacité de travail, des dispositifs doivent assurer une vie décente. Mais cette incapacité doit être démontrée et ne doit en aucun cas être l'effet d'une dépendance aux mesures de redistribution; dans cette logique, la sécurité du revenu ne peut être que minimale et encourager l'insertion professionnelle.

Cette évolution globale s'observe d'ailleurs dans l'inflexion des représentations du chômage, dans le sens d'une individualisation et d'une stigmatisation des sans-emploi. Ainsi, la proportion de la population qui attribue la pauvreté à la paresse ou au manque de volonté passe de $19 \%$ en 1986 à $23 \%$ en 2000 et, parallèlement, la proportion qui considère que cela est dû à l'injustice de la société a diminué de $30 \%$ en 1994 à $21 \%$ en $2000^{16}$. Ce n'est pas encore la situation américaine, mais cela s'éloigne de la culture politique européenne conventionnelle. Rappelons qu'au début des années 1980, plus de la moitié de la population britannique considérait les chômeurs comme des victimes de circonstances

15. Anthony Giddens, The Third Way. The Renewal of Social Democracy, Polity Press, 1998.

16. John Hills, Inequality and the State, Oxford University Press, 2004. 
externes. Cette perception a changé fortement après 1997 avec une importante augmentation dans la proportion de ceux qui estiment que les prestations sont trop généreuses et découragent la recherche active d'emploi ${ }^{17}$.

À ce titre, dans l'environnement européen, la GrandeBretagne s'est fait le porte-étendard de la doctrine de la lutte contre la «dépendance » à l'égard de la protection sociale. Le chômage s'expliquerait par l'abus et l'irresponsabilité, à telle enseigne que les catégories de «chômeur méritant» et de «chômeur non méritant» n'ont pas eu de mal à refaire surface. Déjà les conservateurs s'étaient fait les pourfendeurs de la welfare dependancy, tout en ancrant, dans l'univers de référence, une connotation fortement négative à l'État social et l'acuité du problème de la générosité des prestations et de la fraude de la sécurité sociale ${ }^{18}$. Le New Labour, promoteur d'une «troisième voie», s'engageait à lutter contre le chômage et la pauvreté, en particulier celle des familles de travailleurs. Le gouvernement travailliste cherche à faire la jonction entre un traitement social du chômage et de la pauvreté et un libéralisme économique sur le marché du travail par le développement d'un État social actif qui permet «d'armer les individus face au marché» (selon l'expression de Giddens) ${ }^{19}$. Mais comment ces horizons normatifs se sont-ils traduits dans les politiques publiques?

17. Jochen Clasen, Reforming European Welfare States: Germany and the United Kingdom Compared, Oxford University Press, 2005.

18. Heather Trickey, Robert Walker, «Steps to Compulsion Within British Labour Market Policies», Ivar Lodemel, Heather Trickey (dir.), «An Offer You Can't Refuse»: Workfare in International Perspective, Policy Press, 2001; John Hills, Inequality and the State, Oxford University Press, 2004.

19. Hélène Zajdela, Ai-Thu Dang, Fondements normatifs des politiques d'activation: un éclairage à partir des théories de la justice, Centre d'étude de l'emploi, 2007. 


\section{Le travail comme pivot de la protection sociale}

À la fin des années 1990, un consensus s'est donc développé autour de l'idée d'une politique active et de la légitimité de mesures conditionnelles. Les principaux arguments tablaient sur les coûts associés au nombre de requérants qui n'a pas diminué malgré la croissance de l'emploi, sur le postulat de la dépendance à l'aide sociale, sur la dangerosité et le risque d'agitation sociale spécialement chez les jeunes chômeurs, et sur les histoires à succès des stratégies de workfare américaines et du miracle hollandais ${ }^{20}$. En somme, une part importante du chômage est «volontaire». Pour contrer cette «préférence individuelle», il s'agit de manier à la fois la carotte (par exemple, sous forme de crédits d'impôt en cas de retour sur le marché de l'emploi) et le bâton (diminution puis suppression des prestations pour ceux qui refusent les emplois proposés) ${ }^{21}$.

Pour ce faire, un nouveau contrat a été énoncé entre les citoyens et l'État, par lequel le gouvernement a obligation de fournir des conditions favorisant l'engagement dans un travail rémunéré, alors que les sans-emploi prestataires doivent se montrer proactifs et prêts à accepter formation, travail ou offres d'éducation. Ce contrat s'inscrit dans une nouvelle dynamique des droits et des devoirs (responsabilités) du citoyen-travailleur, en mettant en relief les seconds ${ }^{22}$ : le welfare to work est basé, pour le chômeur, sur un lien entre emploi et protection sociale selon une logique de contre-

2O. Robert Walker, Michael Wiseman, «Making welfare work: UK activation policies under New Labour», International Social Security Review, vol. 56, $\mathrm{n}^{\circ} 1,2003$.

21. Anne Daguerre, «Les politiques de retour à l'emploi aux États-Unis, en Grande-Bretagne et en France», op. cit.

22. Jochen Clasen, Reforming European Welfare States: Germany and the United Kingdom Compared, Oxford University Press, 2005. 
partie $^{23}$. Au-delà de cette conditionnalité de l'aide, notons que la protection sociale destinée aux sans-emploi se caractérise par une forte atténuation de la distinction entre les prestataires de l'assurance et de l'assistance, et par un niveau d'indemnisation "peu généreux», pour employer une litote.

Quelques chiffres permettent d'en prendre la mesure puisque le taux de remplacement net de l'assurance-chômage pour une personne seule est passé de $50 \%$ en 1997 à $46 \%$ en 1999 et de $64 \%$ à $49 \%$ pour un couple avec deux enfants pour la même période ${ }^{24}$. À titre de comparaison, le Royaume-Uni n'a dépensé que $0,19 \%$ du PIB en «mesures passives » en 2005, contre $1,2 \%$ pour la Suède et $2,02 \%$ pour les Pays-Bas ${ }^{25}$. De plus, en proportion des transferts totaux aux chômeurs, la part des prestations financées sur une base contributive a diminué de $45 \%$ en 1979 à $25 \%$ au milieu des années 1980, et de $16 \%$ à la fin des années 1980 à $9 \%$ au cours des années 200o. Il s'agit là d'une indication forte, qui témoigne de la montée en puissance d'une protection pour les chômeurs basée prioritairement sur des conditions de ressources ${ }^{26}$. Par ailleurs, l'Income Support fait office de revenu minimal pour les personnes dans l'incapacité de travailler (avec personne dépendante), malades, handicapées ou aveugles de moins de 60 ans. Versée sous conditions de ressources, cette aide

23. Pascale Dufour, Gérard Boismenu, Alain Noël, L'aide au conditionnel, Presses de l'Université de Montréal, 2003.

24. Jean-Claude Barbier, Analyse comparative de l'activation de la protection sociale en France, Grande-Bretagne, Allemagne et Danemark, dans le cadre des lignes directrices de la stratégie européenne pour l'emploi, DARES, Ministère du Travail, 2006.

25. Nicolas Grivel, Nathalie Georges, Dominique Méda, Les prestations et services d'accompagnement des demandeurs d'emploi: Comparaisons internationales Suède, Pays-Bas, Royaume-Uni, Centre d'étude de l'emploi, 2007.

26. Jochen Clasen, Reforming European Welfare States: Germany and the United Kingdom Compared, op. cit. 
minimale agit comme «filet de sécurité» pour les personnes n'ayant droit à aucune autre allocation, mais n'assure de toute évidence pas la possibilité de se sortir de la pauvreté.

On mesure donc à quel point les questions de travail et de protection sociale deviennent solidaires. L'exclusion sociale est d'abord affaire de mise en marge du travail rémunéré, découlant d'une insuffisance d'éducation, de formation, de compétences ou d'engagement par rapport au marché du travail. Posé en ces termes, le traitement politique de l'exclusion sociale passe par des dispositifs d'activation de ces populations en marge. Il s'agit notamment de développer leur «employabilité» en palliant des handicaps particuliers préjudiciables à leur insertion professionnelle. De là, des mesures de sécurité du revenu conditionnelles et l'introduction de New Deals désignant des situations particulières (parents isolés, adultes handicapés, jeunes de 18 à 24 ans) ${ }^{27}$. Mais cette conception morale de l'exclusion se traduit également par les modalités directives, punitives et disciplinaires des règles qui cadrent les politiques d'activation des prestataires $^{28}$. Dans les Jobcentre Plus, mis en place en 2001, l'essentiel des ressources est alloué au suivi intensif des chômeurs, très peu à la formation et encore moins aux emplois aidés. À la clé, les chômeurs soupçonnés de ne pas remplir leurs obligations de recherche d'emploi peuvent se voir refuser leurs prestations sociales pour une durée pouvant aller jusqu'à 26 mois.

En liant ainsi les questions d'emploi et d'exclusion sociale, le New Labour instrumentalise la citoyenneté. Celle-ci doit

27. Jane Lewis, «New Labour, nouvelle Grande-Bretagne? Les politiques sociales et la "troisième voie" ", Lien social et Politiques, n 41, 1999, p. 61-70.

28. Alexandra Dobrowolsky, Ruth Lister, Social Exclusion and Changes to Citizenship: Woman and Children, Minorities and Migrants in Britain, University of Western, 2005. 
reposer sur une prédominance des responsabilités et des obligations sur les droits et s'accommoder des objectifs économiques et d'injonctions morales: la «grandeur»d'un citoyen se mesure à l'aune de son statut professionnel, mais aussi de son engagement au sein de sa communauté locale. La régulation de l'intérêt collectif s'opère d'abord par l'harmonie de volontés librement consenties. On observe donc un double paradoxe au travers de cette figure du citoyentravailleur. D’une part, la rénovation de l'intervention étatique s'opère par la réactivation de vieux schèmes théoriques, parfois guère éloignés de l'idéal smithien, où la morale individuelle joue un rôle central à côté de la rationalité calculatrice. D'autre part, la responsabilisation des conduites et la rhétorique de l'autonomie sont orchestrées via un ensemble de dispositifs disciplinaires et paternalistes. Pour comprendre l'assise de cette architecture morale, il nous faut mettre en lumière le rôle joué par les principaux acteurs politiques, patronaux et syndicaux. Comment sont régulés collectivement les antagonismes sociaux dans ce système où prime l'échelle de la responsabilité individuelle?

\section{La faiblesse du tripartisme et la marginalisation des syndicats}

Malgré la présence de gouvernements travaillistes dans l'histoire contemporaine de la Grande-Bretagne, le tripartisme, c'est-à-dire la négociation centralisée ou coordonnée des représentants du mouvement syndical, des groupes patronaux et de la direction de l'État, ne s'y est jamais imposé. Perçu avec méfiance de part et d'autre, le tripartisme a cédé la place à la négociation dans les entreprises comme modèle dominant des relations de travail et mode d'élaboration des grands compromis. 
Avec en toile de fond le souvenir exacerbé de conflits de travail fort difficiles au cours des années 1970, le gouvernement Thatcher, cherchant à accroître la compétitivité de l'économie britannique, a disqualifié le mouvement syndical, au point de le considérer comme un ennemi intérieur, et imposé des reculs majeurs dans ses prérogatives d'acteur social $^{29}$. Il suffit de rappeler l'importance des mesures restrictives du thatchérisme visant à mettre le mouvement syndical sur la défensive, à accroître ses frais opérationnels et à réduire sa capacité organisationnelle ${ }^{30}$.

L'emploi est devenu plus concentré dans les services et les nouvelles technologies, engendrant par là une pression pour plus de flexibilité dans les heures travaillées et dans les contrats de travail, et des exigences pour les compétences dans leur acquisition et leur renouvellement ${ }^{31}$. La faiblesse des salaires et des qualifications caractérise le marché du travail en Grande-Bretagne. La stratégie conservatrice après 1979 visait précisément la déréglementation du marché du travail et le soutien à l'entreprise pour maintenir les faibles salaires, non seulement dans le but de lutter contre l'inflation, mais également pour accroître la profitabilité du capital. La coordination des entrepreneurs britanniques étant limitée, ceuxci comptent sur l'État pour être le bras opérationnel de cette entreprise. De faibles salaires devaient s'arrimer à des taux particulièrement bas de remplacement de revenu par la pro-

29. Jochen Clasen, Daniel Clegg, «Unemployment Protection and Labour Market Reform in France and Great Britain in the 1990s: Solidarity Versus Activation?», Journal of Social Policy, vol. 32, n 3, 2003.

30. Alex de Ruyter, John Burgess, «Growing Labour Insecurity in Australia and the UK in the Midst of Job Growth: Beware the Anglo-Saxon Model», European Journal of Industrial Relations, vol. 9, nº 2, 2003.

31. Pete Alcock, Social Policy in Britain, Palgrave-Macmillan, 2003. 
tection sociale, afin d'éviter un télescopage des deux sphères nuisible à l'incitation au travail et au principe du work pays ${ }^{32}$.

À son arrivée à la tête du gouvernement, le New Labour privilégie l'amélioration des droits des individus en emploi. D'où la loi sur le salaire minimum, l'implantation des European Council Directives sur le temps de travail, sur les travailleurs à temps partiel et sur les congés parentaux. Par la suite, au cours du deuxième mandat (2002), l'Employment Act reconnaît davantage de droits (concernant les congés de maternité et de paternité, ou encore le changement des heures de travail), mais son implantation est minimaliste et souffre de délais, blocages et lacunes ${ }^{33}$. Surtout, il s'agit d'une démarche renvoyant à l'octroi de droits individuels pour le travailleur, et non d'une réhabilitation d'une dimension collective de la protection sociale. La séquence travailliste ouverte par le gouvernement de Tony Blair ne signifie pas le retour au premier plan des syndicats ${ }^{34}$. Au contraire, ils seront largement maintenus aux marges du dialogue social par le gouvernement, même si Gordon Brown leur témoignera une écoute plus attentive.

Cette fermeture des néotravaillistes à l'égard des syndicats s'appuie d'ailleurs sur le déclin du nombre de membres syndicaux, enclenché en 1980, qui ralentit à la fin des années 1990, mais continue sa glissade. Le taux de syndicalisation passe de 29,8 à 28,5\% (de 1998 à 2004). Les données qui excluent les petites entreprises donnent des écarts

32. Trine P. Larsen, Anne Daguerre, Peter Taylor-Gooby, Woman and the Labour Market - The National Childcare Strategy Including Sure Start and the Integrated Child Credit, University of Kent, 2001.

33. Anna Pollert, «The Unorganised Worker: The Decline in Collectivism and New Hurdles to Individual Employment Rights», The Industrial Law Journal, vol. 34, $\mathrm{n}^{\circ}$ 3, 2005.

34. Clare Annesley, Andrew Gamble, «Economic and Welfare Policy», Steve Ludlam, Martin J. Smith (dir.), Governing as New Labour. Policy and Politics under Blair, Macmillan, 2004. 
encore plus importants: de $37 \%$ en 1998 à 34\% en 2004. Parallèlement, le nombre de conflits de travail diminue et le pourcentage d'employés liés à des conventions collectives se contracte (il est de moins de $30 \%$ en 2004 et nettement moindre dans le secteur privé, à $21 \%)^{35}$. Là où la négociation collective se poursuit, son champ d'application est plus limité et son impact sur le droit de gestion des entreprises a diminué.

Les relations entre le mouvement syndical (Trades Union Congress) et le New Labour se sont brouillées en raison de la volonté affichée par ce dernier de prendre ses distances et de ne plus être vu comme un partenaire complaisant ${ }^{36}$. La construction du New Labour s'effectue d'ailleurs à rebours de la traditionnelle affiliation des travaillistes au mouvement ouvrier $^{37}$. À l'opposé, la sphère patronale (la Confederation of British Industry), qui a tiré tous les avantages de la politique conservatrice, est de plus en plus alertée par le besoin de formation de la main-d'œuvre et soucieuse que l'intervention publique contribue à une solution. Sur plusieurs sujets, une certaine sympathie patronale se dégage à propos des politiques publiques, comme les New Deals, le salaire minimum, les crédits d'impôt qui supportent le travail mal rémunéré, etc. ${ }^{38}$.

35. William Brown, David Nash, «What has been happening to collective bargaining under New Labour? Interpreting WERS 2004», Industrial Relations Journal, vol. 39, $\mathrm{n}^{\circ}$ 2, 2008, p. 93-96; Anna Pollert, «The Unorganised Worker: The Decline in Collectivism and New Hurdles to Individual Employment Rights», op. cit.

36. Trine P. Larsen, Anne Daguerre, Peter Taylor-Gooby, Woman and the Labour Market - The National Childcare Strategy Including Sure Start and the Integrated Child Credit, op. cit.

37. Florence Faucher-King, «La modernisation du parti travailliste, 1994-2007», Politix, Revue des sciences sociales du politique, vol. 21, n 81, 2008, p. 125-149.

38. Anne Daguerre, Trine P. Larsen, Low Pay and Social Assistance: Minimum Wage and Tax Credits, University of Kent, 2001; Anne Daguerre, Trine P. Larsen, The 1996 Jobseeker's Allowance Act (JSA), University of Kent, 2001. 
On mesure à quel point ce modèle de concorde sociale diffère de l'exemple danois, précédemment exposé. Loin d'une démarche tripartite ou d'instruments de cogestion des dispositifs, on assiste plutôt à la mainmise du gouvernement sur les questions sociales, avec des organes syndicaux affaiblis et tenus à distance. À l'image du gouvernement de Margaret Thatcher, les gouvernements néotravaillistes privilégient surtout les échanges avec des réseaux d'experts et de think tanks. Comme dans le cas français que nous abordons au chapitre suivant, on peut parler d'un resserrement sur des communautés de politiques publiques restreintes, alliant quelques conseillers politiques et de hauts fonctionnaires ${ }^{39}$.

\section{Pauvreté illégitime, inégalités légitimes?}

Au terme de ce parcours, le modèle britannique apparaît très attaché à un individualisme social, à la valorisation de la responsabilité individuelle et à une certaine frugalité dans les dépenses publiques. On observe également une valorisation des valeurs libérales dans l'organisation des relations sociales, la conception de la pauvreté et de l'exclusion sociale, et la façon de penser les dispositifs qui devraient être proposés pour y remédier.

Mais si le travail occupe une place centrale, il n'est pas garant d'une distribution de la richesse qui comble les écarts entre les catégories sociales, ni de réduction de la pauvreté. Il y a un quasi-phénomène de décrochage entre la participation au marché du travail et la distribution de la richesse, avec le double phénomène de généralisation d'emplois précaires et mal rémunérés d'un côté, n'empêchant pas toujours

39. Anne Daguerre, «Les politiques de retour à l'emploi aux États-Unis, en Grande-Bretagne et en France», Critique internationale, n 31, 2006. 
d'éviter la pauvreté, et l'explosion des plus hauts salaires de l'autre $^{40}$. Cela dit, il reste vrai que le changement de la situation de l'emploi est également responsable de l'évolution favorable de la pauvreté: entre 1996-1997 et 2000-2001, il y a eu une diminution de la pauvreté de $1,3 \%{ }^{41}$. Mais ce qui est frappant, c'est la piètre évolution de l'inégalité sociale des ressources et l'état de la pauvreté en Grande-Bretagne, surtout si l'on compare cette situation à celle des pays similaires ${ }^{42}$.

Que l'on considère le coefficient Gini ou la «distance économique»entre les mieux et les plus mal nantis, ces indices témoignent d'un accroissement de l'inégalité dans la distribution de la richesse jusqu'au tournant des années 1990, puis d'une quasi-stagnation, avec un plateau ${ }^{43}$. Sur la séquence 1995-2005, on constate que les personnes les moins nanties ont connu la croissance la plus faible du revenu net, alors qu'à l'opposé, c'est le groupe des plus fortunés qui a eu la croissance la plus forte ${ }^{44}$. Cette croissance de l'inégalité des ressources a pour source l'évolution de la rémunération du travail. En effet, l'évolution des salaires versés aux travailleurs (hommes) au cours des années va dans le sens d'un accroissement de la «distance économique» entre les plus hauts salariés et les plus bas, mais on constate aussi que les

40. Anthony B. Atkinson, Thomas Piketty (dir.), Top Incomes over the Twentieth Century, A Contrast Between European and English-Speaking Countries, Oxford University Press, 2007.

41. Holly Sutherland, Tom Shefton, David Piachaud, Poverty in Britain: The Impact of Government Policy since 1997, Joseph Rowntree Foundation, 2003.

42. Saul Becker, "Security for those who cannot": Labour's neglected welfare principle», Jane Millar (dir.), Understanding Social Security: Issues for Policy and Practice, Policy Press, 2003; David Gordon, Eldin Fahmy, «La pauvreté et l'exclusion sociale en Grande-Bretagne», Économie et statistique, $\mathrm{n}^{\text {os }} 383-385,2005$.

43. Tom Sefton, Holly Sutherland, «Inequality and poverty under New Labour», John Hills, Kitty Stewart (dir.), A More Equal Society? New Labour, Poverty, Inequality and Exclusion, Policy Press, 2005, p. 235.

44. Guy Palmer, Tom MacInnes, Peter Kenway, Monitoring Poverty and Social Exclusion 2006, New Policy Institute, 2006. 
plus hauts salariés connaissent une progression nettement plus avantageuse que celle des salariés au milieu de l'échelle. En somme, la Grande-Bretagne se caractérise par une forte inégalité des revenus, qui ne tend pas à se résorber. Sur le plan de la distribution primaire des revenus comme sur le plan du revenu disponible, c'est-à-dire du revenu tenant compte de l'action des pouvoirs publics, l'inégalité connaît une progression tout en tendant à se stabiliser à des niveaux relativement élevés. Au-delà de la question de la rémunération, c'est également l'accroissement des valeurs immobilières et de patrimoine qui avantage les mieux dotés et pèse sur le budget des moins bien nantis.

Si Gordon Brown a davantage insisté sur la question des inégalités que son prédécesseur Tony Blair, l'accent mis sur des instruments d'incitation à l'activité (en écho aux théories des choix rationnels qui préconisent des systèmes d'incitation et de désincitation face auxquels l'individu orienterait sa conduite) a des avantages, mais aussi d'importantes limites ${ }^{45}$. Ainsi, les crédits d'impôt pour les foyers dotés de revenus d'activité modestes ont permis une légère redistribution pour les $20 \%$ de la population la plus modeste. Mais la plupart des mesures renforcent la priorité donnée à l'égalité des opportunités, plutôt qu'à l'égalité des conditions. À l'image du Child Trust Fund, ce fonds délivré à chaque nouveau-né, qu'il pourra toucher à sa majorité, les mesures de cet «État investisseur» oscillent entre un modèle solidariste (corriger les inégalités de patrimoine) et un modèle responsabilisant (éduquer les pauvres à l'épargne et à la gestion d'un patrimoine $)^{46}$. Surtout,

45. Florence Faucher-King, Patrick Le Galès, Tony Blair 1997-2007, Le bilan des réformes, Presses de Sciences Po, 2007.

46. Pour un retour sur la genèse de cet instrument symptomatique de la «troisième voie», voir Jérôme Tournadre-Plancq, «"Une cuillère en argent pour tout le monde". L'Asset-based Welfare: diffusion, appropriations et usages 
elles apparaissent comme de timides correctifs face au fort accroissement des inégalités de patrimoine ${ }^{47}$.

Si l'on considère non plus les inégalités, mais la pauvreté, il faut tout d'abord noter qu'elle a connu une croissance remarquable au cours des années 1980, tendance aggravée par les politiques néolibérales du gouvernement Thatcher. Avec le tournant des années 1990, on assiste à une certaine stabilisation du phénomène et ce n'est qu'une décennie plus tard que l'on entrevoit une atténuation. Malgré ce léger tassement de la pauvreté de long terme, la situation demeure préoccupante et elle place la Grande-Bretagne au niveau des pays d'Europe du Sud.

La pauvreté est d'abord liée au chômage puisqu'il y a une proportion importante de la population en âge de travailler qui vit dans une famille sans emploi. La propension à être pauvre est cinq fois moindre pour les adultes qui vivent dans un ménage où au moins l'une des personnes travaille. Dans ce dernier cas, le risque de pauvreté reste relativement plus important lorsque c'est seulement l'un des participants au ménage qui travaille et non les deux. Parallèlement, les ménages où la population est dite inactive (ni au travail, ni au chômage, mais soit parent seul, malade ou handicapé) sont le deuxième cas de figure le plus propice à la pauvretét ${ }^{4}$. Cette relation étroite entre pauvreté et relation à l'emploi témoigne

d'une "nouvelle" théorie de l'État social», Revue française de science politique, vol. 59, no 4, 2009, p. 633-653.

47. Soulignons que ce «capital de départ», certes appelé à se développer par l'épargne et des apports extérieurs, est de 250 livres (50o livres pour les enfants de foyers défavorisés) à la naissance de l'enfant, alors que les premiers modèles tablaient sur des dotations de plusieurs dizaines de milliers de livres.

48. Peter Kemp, Jonathan Bradshaw, Paul Dornan, Naomi Finch, Emese Mayhew, Routes out of Poverty. A Research Review, Joseph Rowntree Foundation, 2004. 
GRAPHIQUE 3.1

Pourcentage de la population vivant avec moins de la moitié des revenus moyens (séries $\mathrm{HBAI}$ ), Grande-Bretagne, 1961-2002

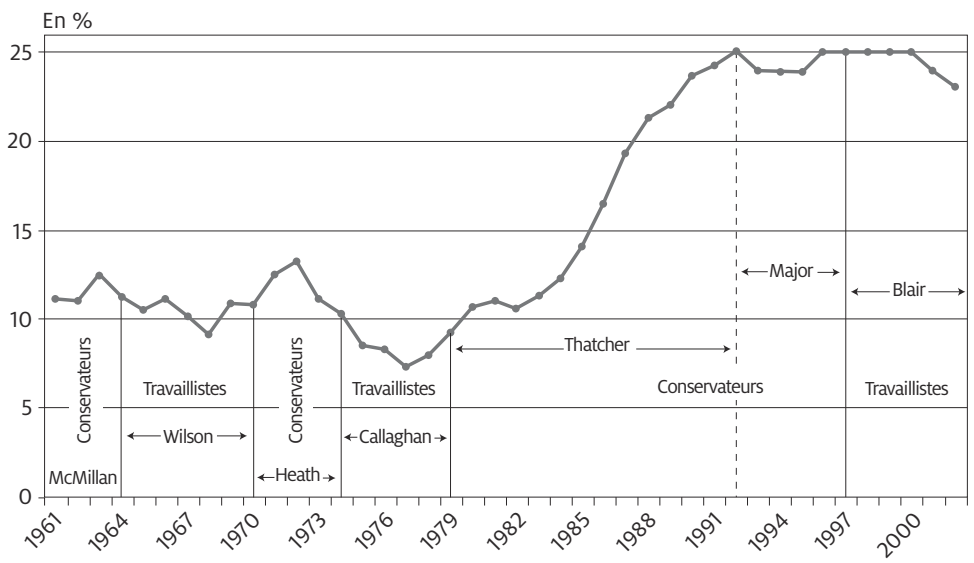

Source: David Gordon, Eldin Fahmy, «La pauvreté et l'exclusion sociale en GrandeBretagne», Économie et statistique, nos 383-385, 2005, p. 125.

GRAPHIQUE 3.2

Taux de pauvreté ( $60 \%$ de la médiane du revenu des ménages) dans les pays européens, 2004

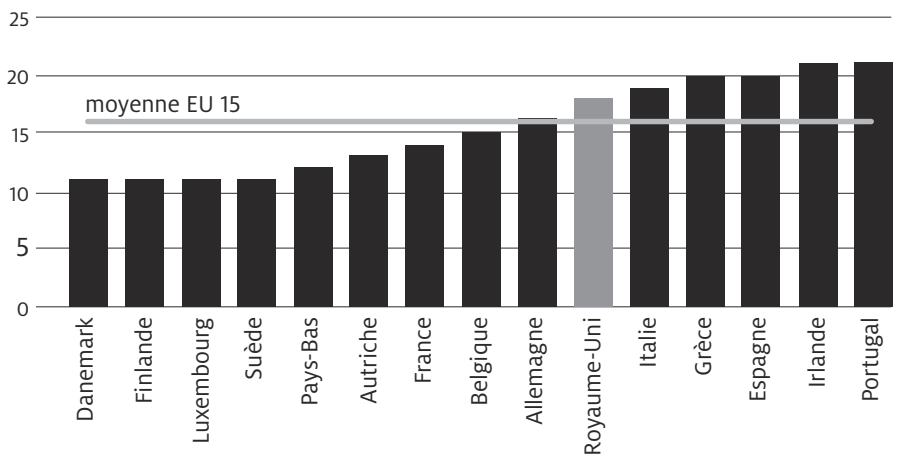

Source: Guy Palmer, Tom MacInnes et Peter Kenway, Monitoring Poverty and Social Exclusion 2006, New Policy Institute, 2006, p. 31. 
également de la faiblesse du niveau de protection publique et particulièrement du revenu de remplacement.

Une observation panoramique révèle d'ailleurs que la situation des sans-emploi au Royaume-Uni est particulièrement préoccupante, si on la met en parallèle avec dix autres pays européens. La proportion des chômeurs (assurancechômage et assistance-chômage) qui sont sous le seuil de pauvreté (60\% de la médiane) est de l'ordre de 51\% en 1996: c'est la pire situation. En 2001, la situation n'a pas évolué. Seules l'Italie et l'Irlande se classent moins bien.

\section{GRAPHIQUE 3.3}

Pourcentage des chômeurs sous le seuil de pauvreté (6o \% de la médiane du revenu normalisé) en 1996 et 2001, onze pays d’Europe

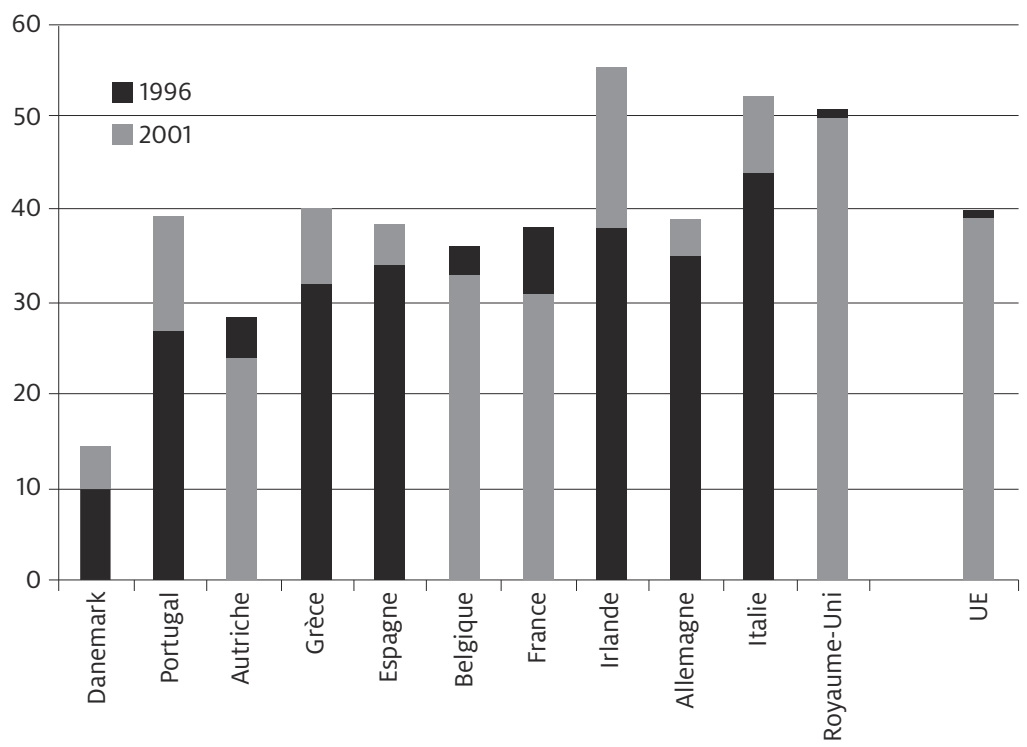

Source: Kitty Stewart, "Changes in poverty and inequality in the UK in international context », John Hills, Kitty Stewart (dir.), A More Equal Society? New Labour, Poverty, Inequality and Exclusion, Policy Press, 2005, p. 317. 
Mais cela ne doit pas masquer le fait que le nombre de ménages qui combinent pauvreté et adulte qui travaille est, toute proportion gardée, de plus en plus important, par rapport à la combinaison pauvreté et ménage sans emploi. Cette articulation entre pauvreté et travail rémunéré est liée à la question de la faiblesse des salaires, notamment pour une part importante de femmes travaillant à temps partiel ${ }^{49}$. C'est seulement en 1999 qu'a été introduite une législation sur le salaire minimum (d'un montant fort modeste) ${ }^{50}$. Elle a eu un impact sur les salaires horaires les plus faibles, pour beaucoup des femmes ${ }^{51}$, mais a faiblement joué sur la réduction de l'inégalité des revenus hebdomadaires parmi les travailleurs pauvres à temps plein ${ }^{52}$.

Parmi ces ménages touchés par la pauvreté, on peut souligner la forte présence de plusieurs types de population. Tout d'abord, la pauvreté touche de plein fouet les enfants. Un coup d'œil sur la situation européenne souligne à quel point le phénomène caractérise la situation britannique: lorsque Tony Blair prend la direction gouvernementale en 1997, le Royaume-Uni occupe la position de queue pour ce qui est du taux de pauvreté des enfants dans l'Europe des 15, celui-ci ayant triplé sur les trois dernières décennies (de 10\% à $33 \%$ ). Le gouvernement du New Labour s'est attaqué à la

49. Guy Palmer, Tom MacInnes, Peter Kenway, Monitoring Poverty and Social Exclusion 2006, op. cit. On peut souligner que les secteurs où est concentré l'emploi féminin (restauration, vente et autres services) sont les secteurs les moins syndiqués et ceux qui pratiquent les plus faibles salaires.

50. Pete Alcock, Social Policy in Britain, Palgrave-Macmillan, 2003.

51. Royaume-Uni. Opportunity for All - Eighth Annual Report 2006: Strategy Document, Department for Work and Pensions, 2006.

52. Holly Sutherland, Tom Shefton, David Piachaud, Poverty in Britain: The Impact of Government Policy since 1997, Joseph Rowntree Foundation, 2003; Trine P. Larsen, Anne Daguerre, Peter Taylor-Gooby, Woman and the Labour Market - The National Childcare Strategy including Sure Start and the Integrated Child Credit, op. cit. 
pauvreté des enfants, avec pour objectif de réduire de moitié le nombre d'enfants touchés de 1998-1999 à 2010-2011 (de 3,4 millions à 1,7 million d'enfants). Malgré des avancées sensibles et des programmes d'envergure, l'objectif d'étape de réduction de $25 \%$ pour 2004-2005 n'a pu être atteint, de même que la projection pour 2010-2011. La situation s'est certes améliorée, mais le pays, tout en cédant la dernière place au Portugal, est toujours dans le peloton de queue.

Ce phénomène se conjugue à celui des travailleurs pauvres puisque la moitié des enfants pauvres vivent dans des ménages où au moins l'un des parents travaille à plein temps ou à temps partiel. Plus encore, le phénomène s'accentue pour les populations issues de l'immigration. Ainsi, $20 \%$ des enfants en situation de pauvreté sont issus des «communautés ethniques minoritaires» et les taux de pauvreté chez les enfants d'Africains subsahariens, de Pakistanais et de Bengalis font plus que doubler les taux des enfants blancs ${ }^{53}$. D'où un phénomène de marginalisation des minorités ethniques qui touche même les personnes nées au Royaume-Uni.

Le revers du modèle socioéconomique britannique, par l'affaiblissement des revenus de protection sociale, pour ne pas «décourager le travail», mais aussi la pression à la baisse des salaires, placent donc une part importante de la population, et notamment les plus fragiles (handicapés, parents seuls, minorités ethniques et individus sans qualification), dans une situation de pauvreté aiguë. Les systèmes d'incitation fiscale et de contrainte administrative afin d'«inciter» les pauvres à «être les entrepreneurs de leur propre réussite» semblent trouver de fortes limites, notamment dans une période où l'offre d'emploi se raréfie.

53. Lisa Harker, Delivering on Child Poverty: What Would It Take?, Secretary of State for Work and Pensions, 2006. 
De plus, le modèle d'activation comporte un revers statistique puisque les données sur le chômage sous-estiment le nombre de personnes sans emploi. En effet, les personnes désœuvrées et découragées sont souvent étiquetées sous la mention de «malades» et échappent aux statistiques sur le chômage, mais n'en sont pas moins présentes dans le contingent des personnes qui sont en marge du marché du travail. Elles comptent dans la masse de la population qui émarge à la protection sociale. D'ailleurs, au tournant des années 2000, sur les quinze pays de l'Union européenne, le Royaume-Uni enregistre le taux d'absence pour maladie le plus élevé au sein de la population en âge de travailler, avec une proportion de $7 \%$, contre seulement 2,1\% en Allemagne et 0,3\% en France. On doit donc considérer que le «véritable» taux de chômage au Royaume-Uni est sans doute supérieur à celui mesuré par le Bureau international du travail. Si l'on devait ajouter aux chômeurs «officiels» les personnes «malades», mais en âge de travailler, le Royaume-Uni arriverait, dans le classement du nombre total de chômeurs, au troisième rang de l'Union européenne à quinze, derrière la Finlande et l'Espagne ${ }^{54}$.

L'exemple britannique souligne à quel point l'articulation vertueuse entre la bonne santé du marché du travail, mesurée par le taux de chômage ou la croissance économique, et la résorption de la pauvreté, mérite d'être questionnée. D’une part, le décrochage entre la participation au marché du travail et la distribution de la richesse affaiblit cette dynamique. De l'autre, les inégalités de revenus ne sont que peu atténuées par l'action redistributrice de l'État. Pour autant, comme on l'a montré, cette situation ne découle pas d'un «raté» du modèle, mais bien d'une architecture morale, de

54. David Gordon, Eldin Fahmy, «La pauvreté et l'exclusion sociale en Grande-Bretagne», Économie et statistique, n ${ }^{\text {os }} 383-385,2005$. 
compromis institutionnels et de politiques publiques, qui concourent à l'entretien de cette situation. In fine, l'ouverture relative $\mathrm{du}$ «cercle des gagnants ${ }^{55}$ », via la mise en place d'instruments censés favoriser l'égalité des chances, prime sur la régulation d'une répartition plus égalitaire des revenus.

Les deux prochains chapitres exposent les situations de la France et du Québec. Au-delà des cas «exemplaires», tels le Danemark et la Grande-Bretagne, ces deux sociétés permettent de cerner avec plus de finesse la diversité des compromis présidant à la destinée de la pauvreté. Inégalités sociales et taux de pauvreté ne sont pas tolérés de manière identique suivant le lieu où l'on se trouve et ne résultent pas des mêmes agencements sociaux-institutionnels, comme nous les reflètent nos quatre cadrans (marché du travail, représentations, traitement politique et compromis sociaux). En France, la segmentation du marché du travail et un taux élevé et constant de chômage se doublent d'une segmentation du traitement politique des situations de pauvreté. Ces rigidités institutionnelles accompagnent une offre de protection sociale qui persiste à mettre de l'avant la solidarité pour les travailleurs et la nécessité d'intervenir sur la répartition des richesses. Au Québec, plusieurs signes montrent une nette amélioration de la situation de la pauvreté ces dix dernières années. Le constat est surtout vrai quand on compare le Québec à lui-même: en revanche, mise en relief dans l'ensemble canadien, la situation québécoise reste fragile. La réduction du nombre de personnes en situation de pauvreté s'est accompagnée d'une persistance des inégalités sociales,

55. On reprend ici le terme d'un promoteur du dispositif du Child Trust Fund. Voir Mark Latham, «Economic Ownership. Expanding the Winners' Circle», Claire Kober, Will Paxton (dir.), Asset-based Welfare and Poverty: Exploring the Case for and against Asset-based Welfare Policies, National Children's Bureau Enterprises, 2002. 
voire d'un accroissement. De plus, à l'image du modèle libéral britannique, la pauvreté est bien souvent associée aux insuffisances du marché de l'emploi. Là aussi, les articulations entre les 4 cadrans nous permettent de comprendre les résultats observés. 



\section{L'idéal solidariste républicain français face à la dualisation du marché du travail}

La France est souvent qualifiée d'inclassable lorsqu'on se situe dans une perspective comparée. On a vu que le regroupement des pays proposé par Pontusson, en termes d'économie sociale de marché (ÉSM) ou d'économie libérale de marché (ÉLM), reléguait la France dans une zone indéfinie, avec l'Italie et le Japon. Il serait tout aussi imprécis d'associer le cas français aux pays d'Europe du Sud. Cette difficulté «classificatoire» met en évidence que l'on retrouve en France une situation qui combine plusieurs influences et perspectives qui l'éloignent des cohérences macrosociologiques que l'on peut percevoir dans certaines sociétés.

On peut ainsi souligner que la France a développé une protection sociale d'inspiration bismarckienne dans le cadre d'un État enclin à un interventionnisme volontariste, tout en étant généralement réfractaire aux formes de concertation néocorporatiste ou assimilée. Économie majeure sur le plan international, elle suit un parcours classique mâtiné d'un souci de protection pour les salariés, de garanties sociales pour les travailleurs âgés, d'un soutien à une politique familiale nataliste et d'une valorisation des droits individuels. 
L'État est garant de la cohésion et de la solidarité nationale. Les dépenses sociales publiques se situent d'ailleurs parmi les plus élevées des pays de l'OCDE, mais les évolutions politiques récentes tendent à modifier cet équilibre. C'est ce cocktail qui rend la configuration française irréductible au libéralisme tout autant que rétive aux compromis institutionnalisés dans lesquels la conciliation/modération des intérêts semble primer.

\section{La pesanteur du chômage de masse}

En termes de richesse produite par habitant, la France se situe parmi les grandes économies, mais n'occupe pas une position dominante ${ }^{1}$. Elle a connu une croissance de la richesse per capita particulièrement élevée de 1960 à 1980, mais le rythme a nettement ralenti dans les deux dernières décennies du $\mathrm{Xx}^{\mathrm{e}}$ siècle. Sur une période plus récente, on peut distinguer une première séquence au cours des années 1995-200O où le PIB a crû en moyenne de 2,8\% par an, d'une seconde, de 2000 à 2005, durant laquelle sa croissance chute à 1,5\% en moyenne. Cette croissance est légèrement supérieure à celle de l'ensemble de la zone euro $(+1,4 \%$ en moyenne par an entre 2000 et 2005) ${ }^{2}$. Par contre, la croissance de la productivité du travail au début des années 2000 est très élevée, se situant au niveau des États-Unis, et nettement supérieure à ce que l'on connaît en Grande-Bretagne. Au même moment, on enregistre en France l'un des taux de chômage les plus élevés, que l'on fasse la comparaison avec

1. Jonas Pontusson, Inequality and Prosperity: Social Europe vs. Liberal America, Cornell University Press, 2005.

2. France. Rapport sur les stratégies pour la protection sociale et l'inclusion sociale 2006-2008, Ministère du Travail, des Relations sociales, de la Famille et de la Solidarité, 2006, annexe. 
les pays d'économie libérale de marché ou d'économie sociale de marché.

À ce titre, il faut souligner à quel point le chômage suit un parcours accidenté depuis plus d'une décennie. Après une contraction à la fin des années 1980, il connaît une forte hausse au début des années 1990, qui se prolonge pendant quelques années. Puis, un léger tassement se confirme dans les années 2000 (9,7\% en 2005). Cette évolution est singulière par rapport aux autres pays abordés dans notre étude. Ainsi, au cours des années 1990, la crise dont souffre la France n'est pas partagée par plusieurs pays. En 2005, le taux de chômage à 9,7\% en France est supérieur d'un point de pourcentage à la moyenne de l'Europe à 25: en fait, mis à part l'Allemagne, la situation de l'emploi est nettement plus difficile en France que dans les pays d'économie sociale de marché nordique, comme le Danemark, ou des pays d'économie libérale de marché, comme la Grande-Bretagne.

En termes de structure de la population au chômage, la France se distingue également. Ainsi, les femmes sont plus touchées par le chômage que les hommes; il est vrai que l'écart se réduit depuis quelques années, mais on ne peut ignorer que dans certains pays cet écart n'existe pas ou est inverse. De plus, le chômage est vécu différemment selon les strates d'âge. Nettement plus sévère pour les jeunes de 15 à 24 ans, alors que plus de 20 \% sont au chômage en 2005, le taux de chômage n'est plus qu'à 9,0\% pour les 25-49 ans et $6,7 \%$ pour les 50 ans ou plus. L'incidence de l'éducation et de la formation générale ne fait pas de mystère: plus la formation est poussée, moins le chômage sévit ${ }^{3}$. Comme

3. Contrairement à ce que suggère l'hypothèse de la perte de valeur des diplômes, Éric Maurin démontre au contraire leur poids de plus en plus déterminant à mesure que la situation économique se dégrade. Voir Éric Maurin, La peur du déclassement, une sociologie des récessions, Seuil, 2009. 
le souligne le rapport de l'Insee, les fortes disparités entre catégories socioprofessionnelles se maintiennent puisqu'en 2005 le chômage est presque quatre fois plus fréquent chez les ouvriers non qualifiés $(18,8 \%)$ que chez les cadres $(4,9 \%)^{4}$.

Sur un autre plan, il faut noter que les populations issues de l'immigration sont nettement plus concernées par le chômage, et ce, à tous les titres. Il s'agit d'un «facteur aggravant» qui se décante de la façon suivante: pour tous les groupes d'âge, le fait d'être un homme immigré ${ }^{5}$ augmente l'incidence du chômage et le fait d'être une femme l'augmente encore. Cela signifie que dans tous les cas de figure, en termes de strates d'âge, par exemple, la population immigrée, homme et, encore davantage femme, sera plus touchée que l'ensemble de la population.

Mais la spécificité la plus notable du chômage en France, au regard des autres configurations étudiées, est l'importance du chômage de longue durée. Alors qu'en 2002, il est de près de $1 \%$ au Danemark et au Royaume-Uni, le chômage de longue durée (de plus d'un an) atteint 2,8\% en France. Seule avec la Belgique, la France est à peu près à la moyenne (3\%) de l'UE à 15 pays, alors que les pays d'Europe du Sud ont une situation beaucoup plus aggravée. Le problème du chômage de longue durée s'est approfondi depuis en France, alors que les taux se sont stabilisés autour de $4 \%$ en 2005. Les mesures actives du marché du travail, qui visaient directement ce type de sans-emploi, n'ont pu redresser la situation: la proportion des chômeurs de longue durée parmi l'ensem-

4. France, portrait social.Édition 2007 , Institut national de la statistique et des études économiques, 2007.

5. Un individu vivant en France est considéré comme «immigré» s'il est né à l'étranger et ne possédait pas la nationalité française à sa naissance. Après son arrivée en France, il peut devenir français par acquisition (immigré français) ou garder sa nationalité (immigré étranger). 
TABLEAU 4.1

Taux de chômage en 2005 des personnes immigrées et non immigrées selon le sexe et l'âge (en \%)

\begin{tabular}{|l|c|c|c|c|}
\hline & Ensemble & $\begin{array}{c}\mathbf{2 5} \text { à } \mathbf{3 9} \\
\text { ans }\end{array}$ & $\begin{array}{c}\mathbf{4 0} \text { à } \mathbf{4 9} \\
\text { ans }\end{array}$ & $\begin{array}{c}\mathbf{5 0} \text { ans } \\
\text { ou plus }\end{array}$ \\
\hline Hommes actifs immigrés & 15 & 17 & 14 & 14 \\
Hommes actifs non immigrés & 8 & 9 & 5 & 6 \\
Ensemble des hommes actifs & 9 & 9 & 6 & 7 \\
\hline Femmes actives immigrées & 22 & 28 & 18 & 16 \\
Femmes actives non immigrées & 10 & 11 & 7 & 6 \\
Ensemble des femmes actives & 11 & 12 & 8 & 7 \\
\hline Ensemble de la pop. active immigrée & 18 & 21 & 16 & 15 \\
Ensemble de la pop. active non immigrée & 9 & 10 & 6 & 6 \\
Population active totale & 10 & 11 & 7 & 7 \\
\hline
\end{tabular}

Source: France. Rapport sur les stratégies pour la protection sociale et l'inclusion sociale 20062008, Paris, Ministère du Travail, des Relations sociales, de la Famille et de la Solidarité, 2006, annexe p. 10.

ble des chômeurs se situe au-delà des $40 \%$. Le cinquième des chômeurs sont dans cette situation durant une période qui dépasse les deux ans ${ }^{6}$. À ce titre, la situation française se démarque assez nettement de la tendance dans la zone euro ainsi que de l'ensemble des pays de l'OCDE. Notons que ce chômage de longue durée touche davantage les femmes que les hommes $(43,2 \% \text { contre } 41,8 \%)^{7}$. De plus, la situation est très critique pour les 50 ans et plus.

En ce qui concerne son taux d'activité, la France se situe dans la moyenne européenne, avec une forte progression, en termes relatifs, depuis une dizaine d'années. L'écart entre les hommes et les femmes tend notamment à se rétrécir. Cependant, le taux d'activité générale est très inférieur à ce qu’on

6. Jochen Clasen, Daniel Clegg, «Unemployment Protection and Labour Market Reform in France and Great Britain in the 1990s: Solidarity Versus Activation?», Journal of Social Policy, vol. 32, n 3, 2003.

7. Institut national de la statistique et des études économiques, France, portrait social. Édition 2007. 
TABLEAU 4.2

Taux d'emploi en 2008, par âge, dans l'Union européenne (en \%)

\begin{tabular}{|l|c|c|c|c|}
\hline & $\mathbf{1 5}$ à 24 ans & $\mathbf{2 5}$ à 54 ans & 55 à 64 ans & Ensemble \\
\hline Danemark & 67,0 & 88,0 & 57,0 & $\mathbf{7 8 , 1}$ \\
\hline France & 32,2 & 83,2 & 38,3 & $\mathbf{6 5 , 2}$ \\
\hline Royaume-Uni & 52,4 & 81,4 & 58,0 & $\mathbf{7 1 , 5}$ \\
\hline UE à 27 & $\mathbf{3 7 , 6}$ & $\mathbf{7 9 , 6}$ & $\mathbf{4 5 , 6}$ & $\mathbf{6 5 , 9}$ \\
\hline
\end{tabular}

Source: Eurostat, 2009.

observe au Danemark ou au Royaume-Uni, en raison du statut spécifique des populations en amont et en aval du marché de l'emploi. On désigne ainsi d'une part les jeunes, dont la scolarité est relativement longue et l'entrée sur le marché du travail plus tardive - et ardue, nous y reviendrons - que dans la plupart des pays européens. Mais d'autre part, ce sont les franges âgées de 55 à 64 ans qui sont beaucoup moins présentes sur le marché de l'emploi que leurs vis-à-vis britanniques et surtout danois. Cela est en partie lié aux politiques d'éviction précoce des travailleurs plus âgés par des modalités de préretraite et retraite avantageuses. L’âge légal de la retraite y est d'ailleurs de 60 ans (avant les réformes de 2010), contre 65 au Royaume-Uni et 67 au Danemark. Plus qu'ailleurs, les emplois sont monopolisés par les travailleurs de 25 à 54 ans.

\section{La monopolisation par l'État de l'intérêt général}

L'État français est historiquement porteur d'une mission inspirée par la pensée républicaine qui invite à la solidarité. La pauvreté et l'exclusion sociale sont vécues comme l'expression d'une rupture des liens sociaux, d'où cette solidarité qui renvoie à un pacte social, à une communauté morale et à une politique pétrie des droits, des valeurs et des obliga- 
tions. Garant de la cohésion sociale, l'État doit intervenir pour rétablir l'ordre, notamment par des institutions et des politiques. Le politique est nécessairement à l'avant-scène, tant du point de vue du gouvernement que des acteurs collectifs. Ainsi, il constitue le système de référence par lequel se pense et se projette le traitement de la pauvreté et de l'exclusion sociale.

Le monopole légitime de la prise en charge de l'intérêt général par l'État, si l'on peut dire, reconduit les injonctions d'un modèle républicain et centralisateur qui fait une faible place à la reconnaissance des corps intermédiaires, quelle que soit la forme prise par l'organisation des intérêts. À ce titre, on peut souligner le volontarisme de l'État dans l'encadrement des relations de travail et surtout dans la fixation de «standards» nationaux. Le modèle de pratiques qui caractérise la France est influencé davantage par le pluralisme que par la stratégie de la coopération entre les acteurs ${ }^{8}$.

De son côté, l'histoire singulière du syndicalisme ouvrier français et sa fragmentation l'opposent aux modalités de concorde sociale de type néocorporatiste. Le mouvement syndical jouit d'une force politique et d'une capacité d'impact nettement supérieures à son poids dans l'organisation des travailleurs: le taux de syndicalisation est faible, le mouvement syndical est fragmenté et le pouvoir des confédérations sur leurs éléments est fragile. De plus, ce syndicalisme peine à s'implanter dans les bassins d'emploi en fort développement, où priment CDD, intérim et autres formes d'emploi dites atypiques, peu propices à l'organisation d'une

8. Gérard Boismenu, «Systèmes de représentation des intérêts et configurations politiques», Revue canadienne de science politique, vol. 27, $\mathrm{n}^{\circ}$ 2, 1994, p. 309-343; Gérard Boismenu, «Modèles politico-institutionnels et politique macro-économique. Analyse comparée de douze pays industrialisés, 19601988 », Études internationales, vol. 26, n² 2, 1995, p. 237-274. 
mobilisation. Une telle situation serait, dans d'autres pays, le témoignage de la marginalisation de cet acteur collectif. Or, la grande politisation du traitement des enjeux en fait, au contraire, un interlocuteur obligé, même sous des formes plus ou moins conflictuelles.

De l'autre côté de l'espace social, la centrale des patrons, le MEDEF, recomposée en octobre 1998, s'engage dans une «refondation sociale» avec un succès mitigé. Celle-ci vise à «déplacer» le dialogue social de la scène nationale vers l'entreprise, voire au besoin à la branche industrielle. Façon de dépolitiser les enjeux et les conflits, le nouveau dialogue social devrait se tenir sans l'État, sauf lorsque cela s'avère tout à fait nécessaire, en valorisant la négociation décentralisée ${ }^{9}$. Dans le domaine de la protection sociale, le patronat n'a pas nécessairement réussi à imposer ses vues, mais il a pu procéder à un recadrage des thématiques et des enjeux, ce qui n'est pas négligeable. Les «dirigeants du MEDEF ont réussi [environ en 2000] avec la refondation sociale [...] à déplacer le débat sur le terrain des thèmes chers aux approches libérales de la protection sociale: poids économique et inefficacité sociale des systèmes publics de protection sociale, incitations/désincitations au travail, liées aux prestations sociales, thématiques de la responsabilité individuelle, de l'activation des dépenses sociales, de la transformation de la nature des risques ${ }^{10} \ldots$ » On reconnaît là un certain nombre de thématiques rencontrées outre-Manche (et a fortiori outre-Atlantique). Ce déplacement discursif constitue une

9. Jacques Freyssinet, «La réforme de l'indemnisation du chômage en France», La Revue de l'IRES, $\mathrm{n}^{\circ}$ 38, 2002.

10. Bruno Palier, Gouverner la sécurité sociale: Les Réformes du système français de protection sociale depuis 1945, Quadrige/Presses universitaires de France, 2005, p. 411. 
pièce majeure pour tout changement d'orientation dans le domaine des politiques publiques.

\section{Intolérance (théorique) et accommodements (pratiques) envers la pauvreté}

L'idéologie républicaine semble définir un seuil de tolérance appelant les pouvoirs publics à juguler la pauvreté, car elle est perçue comme une défaillance de l'ordre social et un défi à la solidarité nécessaire sous l'égide de la république. Dès la Révolution, la France a donné force à la notion de citoyenneté en postulant que l'individu devait être intégré au corps social et en en faisant un enjeu politique ${ }^{11}$. La fraternité appelle la solidarité, mais celle-ci prend des visages différents selon les époques. Dans la constitution de 1946, la poursuite de l'égalité est associée à l'intervention publique, mais la protection doit composer avec les formes institutionnelles empreintes des particularismes «corporatistes», d'où la combinaison de principes universalistes et de la diversité des droits en fonction des activités professionnelles ${ }^{12}$. Dans sa construction même, le système de sécurité sociale français induit une différenciation forte des traitements des travailleurs, en fonction de leur corporation, puis en fonction de leur statut d'emploi. La segmentation croissante du marché du travail, liée à la multiplication des types de contrats de travail, va aller de pair avec une segmentation du traitement des personnes sans emploi. Ainsi, les rigidités souvent

11. Michel Dollé, «La décentralisation du RMI et la création d'un revenu minimum d'activité, le RMA: une réforme problématique», Droit Social, $\mathrm{n}^{\mathrm{os}} 7-8,2 \mathrm{OO}$.

12. Bruno Jobert, «De la solidarité aux solidarités dans la rhétorique politique française», Colette Bec, Giovanna Procacci, Claudine AttiasDonfut (dir.), De la responsabilité solidaire: mutations dans les politiques sociales aujourd'hui, Syllepse, 2003, p. 77-78. 
évoquées à propos du «modèle» français sont autant liées à des compromis historiquement construits entre l'État et les «partenaires sociaux» qu'aux positionnements des syndicats pour la défense des droits acquis dans la période récente.

Alors que le système de sécurité sociale (composé de régimes assuranciels et d'allocations universelles) devait repousser la pauvreté à la marge de même que les politiques d'assistance, la réapparition brutale d'une nouvelle pauvreté, à la faveur des crises des années 1970, a provoqué l'émergence de mécanismes assistanciels.

L'émergence de la notion d'exclusion dans les années 1980 lie le chômage à un déficit d'intégration. L'insertion devient alors le maître mot des politiques sociales. La «nouvelle pauvreté», qui se distingue d'une pauvreté perçue comme «marginale», prend les traits d'une pauvreté «disqualifiante ${ }^{13}$ », pouvant toucher tout le monde. Face à ce fléau, on observe une réaction ambivalente de l'opinion et des pouvoirs publics en France, où les injonctions républicaines se teintent de compassion et de mauvaise conscience, à l'inverse des logiques individualisantes et punitives du système britannique. Simonin rappelle avec à propos l'interprétation de Donzelot voulant que l'on «considère en France [...] que tout problème social de l'individu est d'abord de la faute de la société, que l'individu subit les effets de la société, que celle-ci donc lui doit protection, et que si cette dernière vient à manquer, il se trouve comme exclu, rejeté par une société qui contracte pour le coup une dette envers lui, celle de le faire vivre [...] de lui permettre d'éprouver le sentiment de son utilité sociale, de trouver à l'employer pour lui assurer

13. Pour un retour sur cette distinction, voir Serge Paugam, Les formes élémentaires de la pauvreté, PUF, 2005. 
[...] sa propre cohésion ${ }^{14} »$. On est là bien loin de la rhétorique du blairisme, et encore davantage du thatchérisme.

Pour autant, des évolutions récentes méritent d'être soulignées, pour prendre la mesure du brouillage de ces repères normatifs. On peut reprendre l'hypothèse de Bruno Palier ${ }^{15}$ à propos d'une dualisation de la protection sociale. D'un côté, on assiste au développement depuis les années 1970 de minima sociaux, dont bénéficient les individus démunis (en 2008 , près de $10 \%$ de la population française ${ }^{16}$ ), au nom de la solidarité nationale. De l'autre, un ensemble de dispositifs ont été introduits, reposant sur le modèle de l'activation. C'est le lien entre ces deux versants des politiques sociales qui nous intéresse. À la fin des années 1990 apparaît dans le débat politique français une explication de l'inactivité qui tranche avec celle jusqualors dominante: des minima sociaux trop hauts, comparés à des emplois faiblement rémunérés, décourageraient la reprise d'activité. Un rapport de l'Insee appuie cette hypothèse de la désincitation au travail et introduit les termes du débat anglo-saxon, de la «désincitation» (disincentive effect) à la «trappe à pauvreté» (poverty trap).

Ces préceptes mettent l'accent sur le «chômage volontaire» et donc sur l'individualisation du problème. La solution passerait par des incitations afin de moduler le calcul coûtavantage du chômeur entre la «tentation du chômage» et la «préférence pour l'emploi». Même si ces préceptes vont à

14. Bernard Simonin, «Indemnisation, placement, activation: la politique de l'emploi tiraillée entre plusieurs modèles de responsabilité», Colette Bec, Giovanna Procacci, Claudine Attias-Donfut (dir.), De la responsabilité solidaire: mutations dans les politiques sociales aujourd'hui, Syllepse, 2003, p. 127.

15. Bruno Palier, Gouverner la sécurité sociale. Les réformes du système français de protection sociale depuis 1945, Quadrige/Presses universitaires de France, 2005.

16. Bruno Palier, «Du welfare au workfare: les transformations des politiques de lutte contre la pauvreté», Regards croisés sur l'économie, La Découverte, $n^{\circ} 4,2008$. 
l'encontre de la vision républicaine d'un traitement collectif du chômage, ils trouvent néanmoins plusieurs points d'appui dans le débat hexagonal.

D'abord, soulignons le travail de promotion de ces idées par des réseaux d'experts, mêlant démonstration microéconomique et considérations morales, et s'instituant en relais des préconisations de l'OCDE, de l'Union européenne (Sommet de Lisbonne en 2000), mais aussi d'expériences étrangères, notamment les pays anglophones ${ }^{17}$.

Ensuite, il faut préciser le contexte favorable, avec des années de reprise de la croissance, qui voit le taux de chômage décroître. Or, ce type de configuration est favorable au succès d'une explication du chômage par la rhétorique de la paresse. C'est ce que mettent en lumière Serge Paugam et Marion Selz en étudiant l'évolution depuis le milieu des années 1970 de deux matrices explicatives à propos du chômage ${ }^{18}$, cadré soit comme une injustice au sein de la société (et c'est alors un problème collectif), soit comme une mauvaise volonté de la part du chômeur (et c'est le devoir de celui-ci de «s'activer» et de «se mettre au travail»). Au-delà des différences nationales, ils observent une progression générale de l'explication par la paresse entre 1993 et 2001. Comme le précise S. Paugam: «Lorsque le chômage diminue, la probabilité de donner cette explication augmente fortement, ce qui s'est effectivement produit à la fin des années 1990. Tout se passe comme si la population considérait, en période de reprise de l'emploi, que, si les pauvres ne trou-

17. Pour un retour sur ce travail d'importation, notamment sous la férule d'économistes de l'Insee, voir Pierre Strobel, «Les chômeurs sont-ils responsables de leur sort?», Lien social et Politiques, n 46, 2001, p. 163-173.

18. Serge Paugam, Marion Selz, «La perception de la pauvreté en Europe depuis le milieu des années 1970. Analyse des variations structurelles et conjoncturelles», Économie et statistique, nº 383-385, 2005, p. 283-305. 
vaient pas d'emploi, c'était au moins en partie de leur faute ${ }^{19}$.» Cela se répercute en France sur la perception du RMI (revenu minimum d'insertion), dont les bénéficiaires passent progressivement dans l'opinion publique du statut de victime à celui de privilégié2 ${ }^{20}$. Pour Nicolas Duvoux, la mise en concurrence, dans le débat politique entre «les assistés» et «les travailleurs à bas salaires», a été orchestrée par une exploitation médiatique et politique, dans laquelle a joué l'amalgame avec la question de l'immigration ${ }^{21}$.

S’il eut jusqu'au début des années 2000 un succès mitigé dans l'opinion publique ${ }^{22}$, ce jeu d'opposition entre les «profiteurs» et les «méritants», entre «eux» et «nous», prend un visage particulier en France puisque la stigmatisation des autres s'articule à une peur largement partagée de «tomber» dans la pauvreté. Alors que le taux de pauvreté y est plus faible, les enquêtes d'opinion montrent paradoxalement que la perception du risque de pauvreté, et le sentiment que la pauvreté puisse toucher un jour ou l'autre, se situent à un niveau nettement plus élevé en France que dans le reste de l’Europe $^{23}$. Cette énigme renvoie à la structure spécifique du marché du travail en France, avec un centre intégré, aux p. 60 .

19. Nicolas Duvoux, Serge Paugam, La régulation des pauvres, PUF, 2008,

20. David Alibert, Régis Bigot, Le regard des Français sur les politiques de l'emploi, le chômage, la formation et le RMI, Centre de recherche pour l'étude et l'observation des conditions de vie, 2006.

21. Nicolas Duvoux, Serge Paugam, La régulation des pauvres, op. cit. Voir aussi Mourad Guichard, «France: Nicolas Sarkozy et le "chômeur-profiteur" imaginaire», Libération, 21 mai 2008.

22. Bruno Jobert, «De la solidarité aux solidarités dans la rhétorique politique française», Colette Bec, Giovanna Procacci, Claudine Attias-Donfut (dir.), De la responsabilité solidaire: mutations dans les politiques sociales aujourd'hui, Syllepse, 2003.

23. Stéphanie Jamet, Lutter contre la pauvreté et l'exclusion sociale en France, Document de travail du Département des Affaires économiques, Éditions OCDE, 2007, p. 7-8; Éric Maurin, La peur du déclassement, une sociologie des récessions, op. cit. 
emplois bien protégés, et une marge en expansion où se développent des formes précaires d'emploi. Celles-ci occupent une situation à l'interface du monde du travail intégré et du chômage de longue durée, dont on a vu l'importance en France. On aborde donc là deux éléments clefs: la croissance des «travailleurs pauvres» et l'explosion des formes d'emploi dites «atypiques». Cette reconfiguration du marché du travail et des risques de pauvreté conduit à mettre en question les politiques publiques qui ont été mises en place sur ces thématiques.

\section{La dualisation du marché du travail : vers un workfare à la française?}

L'emploi est de moins en moins un antidote à la pauvreté, comme l'indique l'émergence du phénomène des «travailleurs pauvres». Parmi les adultes en âge de travailler, qui sont en situation de pauvreté, $41 \%$ occupent un emploi. Ces travailleurs pauvres représentent $6,4 \%$ de la population active française en 2005, alors que ce taux n'est que de $1 \%$ au Danemark $^{24}$. Surtout, c'est l'évolution de leur nombre qui inquiète. Ainsi, sur une séquence de reflux du chômage importante (1996-2OO1), leur nombre baisse faiblement, mais dans une séquence plus terne (2003-2005), leur nombre croît de $25 \%{ }^{25}$. Or, leur croissance doit être mise en lien avec un ensemble de politiques publiques qui ont poussé à la déréglementation des marchés du travail et, plus largement, à la dualisation du marché du travail. Traditionnellement, la protection des emplois réguliers en France est une des plus importantes en Europe et le coût du travail un des plus éle-

24. Yannick L'Horty, «Pourquoi le travail ne protège plus de la pauvreté», Regards croisés sur l'économie, vol. 2, n 4, 2008, p. 107-114.

25. Idem. 
vés, tout comme le montant du salaire minimum ${ }^{26}$. Cette politique permet de lutter contre la pauvreté, mais pénalise l'emploi très peu qualifié et de faible productivité, ce qui devient une rigidité pour l'insertion sur le marché du travail. On a donc vu depuis le début des années 1990 le développement des nouveaux contrats dérogeant à la norme du contrat à durée indéterminée, avec diminution des charges sociales et des cotisations. Ces formes d'emploi persistent à être nommées «atypiques» alors qu'elles deviennent la norme: elles concernent les trois quarts des contrats de travail passés dans les dernières années, que ce soit sous forme d'emploi à durée limitée (CDD), d'intérim ou de travail à temps partiel ${ }^{27}$.

Cette destitution du contrat à durée indéterminée, bien encadré et réglementé, comme canon du modèle d'embauche, ne va pas de soi, comme l'ont montré des mouvements sociaux d'ampleur, par exemple contre le Contrat de première embauche (CPE) en 2006. Ces mesures ont eu un impact positif sur l'emploi, notamment par la diminution des charges sociales et cotisations patronales, mais cet effet quantitatif s'est accompagné d'une augmentation forte et régulière d'emplois au bas de l'échelle et peu protégés, sous forme d'intérims, de contrats à durée déterminée, de contrats d'apprentissage, de stages et de contrats aidés. Tandis que la rigidité de l'emploi demeure avérée dans les segments les plus traditionnels du marché du travail, dans d'autres on assiste à une nette poussée de flexibilisation de la contractualisation de l'emploi. À rebours du modèle danois, la flexibilité rime ainsi avec le sentiment d'insécurité. Cette situation met au défi l'univers de représentation républicain qui insiste sur

26. Stéphanie Jamet, Lutter contre la pauvreté et l'exclusion sociale en France, op. cit.

27. Robert Castel, La montée des incertitudes. Travail, protections, statut de l'individu, Seuil, 2009. 
le devoir de solidarité, s'exprimant jusqu'ici par l'encadrement légal du travail et la protection sociale, afin d'assurer la sécurité des citoyens dans leur participation à la sociétée ${ }^{28}$. Les politiques publiques concernant l'emploi trouvent alors des points d'articulation problématiques, voire de tension, avec les politiques sociales.

En effet, le système de protection sociale en France a été mis en place avec l'idée de répondre aux difficultés passagères des citoyens, causées par les risques sociaux, et non pour faire face de façon durable à une pauvreté endémique ou pour corriger des difficultés structurelles nuisant à l'insertion sur le marché du travail ${ }^{29}$. Le système de protection sociale fait référence traditionnellement à un marché du travail formalisé où les travailleurs (masculins) sont pourvoyeurs de ressources pour la famille, bénéficiant de droits et de garanties sur une société caractérisée par l'assurance sociale. Dans ce modèle, une force de travail composée de jeunes, de personnes âgées, de femme, etc. qui ne peuvent tirer les mêmes bénéfices et ne sont pas porteurs des mêmes droits. Cette segmentation mène tendanciellement à une situation où les premiers sont interconnectés à un système assuranciel et les seconds à un système assistanciel, voire à la garantie de minima sociaux ${ }^{30}$. Cette segmentation et les droits qui y sont attachés se complètent de l’héritage de

28. Jean-Claude Barbier, Bruno Théret, Le nouveau système français de protection sociale, La Découverte, 2004; Jean-Claude Barbier et al., Normative and Regulatory Frameworks Influencing the Flexibility, Security, Quality and Precariousness of Jobs in France, Germany, Italy, Spain and the United Kingdom, ESOPE Project, European Commission.

29. Michel Borgetto et al., Les débats sur l'accès aux droits sociaux entre lutte contre les exclusions et modernisation administrative, Centre d'études et de recherches de Sciences administratives et politiques (CERSA), 2004.

30. Bruno Palier, Christelle Mandin, Policy Maps - France, University of Kent, 2003. 
politiques familiales ${ }^{31}$ et d'une protection sociale contributive, liée aux corps d'emploi tout en étant généralisée, touchant aux risques pour la santé et pour la vieillesse ${ }^{32}$.

À la fin des années 1990, face à un chômage qui ne semble pas devoir se résorber, à la persistance de la pauvreté et de formes diverses d'exclusion, la loi contre les exclusions (1998) souligne que le devoir de solidarité mène à une politique d'assistance, tout en veillant à sortir les citoyens de leurs difficultés, d'abord par l'accès à l'emploi et à des conditions de vie décentes ${ }^{33}$. Cela signifie de chercher à réduire le chômage, à augmenter le taux d'activité de la population et à lutter contre les difficultés graves d'insertion de certaines populations. Pour résumer, l'action publique fut déployée dans trois directions: développement de l'offre d'activités et d'emplois; incitation à la sortie des logiques d'assistance et à la reprise d'emploi via la sécurisation des parcours professionnels; et enfin la facilitation de la participation au marché du travail par une levée des obstacles à l'entrée ou au retour à l'emploi ${ }^{34}$. Les populations, dans leurs caractéristiques propres, revêtent dès lors plus d'importance et appellent à des traitements différenciés. Cependant, le ciblage reste faible puisque de nombreuses dépenses (retraite, santé, politiques familiales) ne sont pas placées sous conditions

31. Jean-Claude Barbier, Valeria Fargion, «Continental Inconsistencies on the Path to Activation: Consequences for Social Citizenship in Italy and France», European Societies, vol. 6, $\mathrm{n}^{\circ}$ 4, 2004.

32. France. Le rapport de l'Observatoire national de la pauvreté et de l'exclusion sociale 2007-2008, Observatoire national de la pauvreté et de l'exclusion sociale, La Documentation française, 2008.

33. Michel Borgetto et al., Les débats sur l'accès aux droits sociaux entre lutte contre les exclusions et modernisation administrative, Centre d'études et de recherches de Sciences administratives et politiques (CERSA), 2004.

34. France. Rapport sur les stratégies pour la protection sociale et l'inclusion sociale 2006-2008, Ministère du Travail, des Relations sociales, de la Famille et de la Solidarité, 2006. 
GRAPHIQUE 4.1

Dépenses pour l'emploi en France

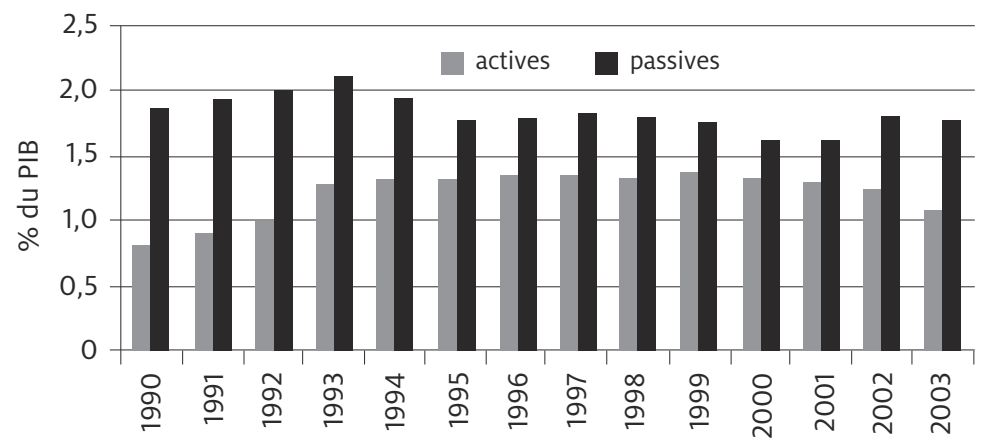

GRAPHIQUE 4.2

Dépenses pour l'emploi au Royaume-Uni

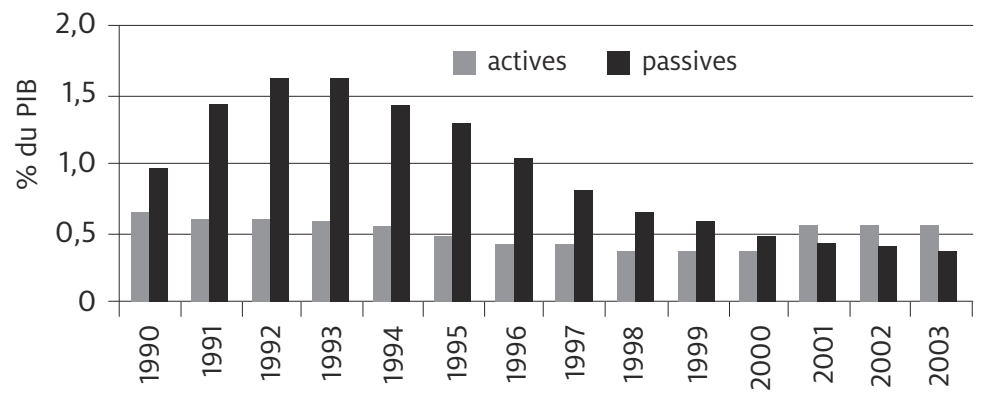

Source: Anne Eydoux, «Des sociétés pleinement actives? Marchés du travail et protection sociale, une analyse quantitative transversale», Jean Claude Barbier (dir.), Analyse comparative de l'activation de la protection sociale en France, Grande-Bretagne, Allemagne et Danemark, dans le cadre des lignes directrices de la stratégie européenne pour l'emploi, DARES, Ministère du Travail, 2006, p. 158. 
de ressources ${ }^{35}$. On voit donc se mettre en place une version hybride des politiques d'activation, entre héritage universaliste et injonctions au ciblage, démarches assurantielles et assistancielles, compassion et surveillance des bénéficiaires. Si l'on compare l'évolution des dépenses actives et passives en France et au Royaume-Uni, on mesure bien, au-delà des parts du PIB qui y sont consacrées, des répartitions très différentes. Ainsi, les dépenses dites passives restent importantes en France, alors qu'elles sont divisées par quatre durant la même séquence, outre-Manche.

Même si la France adhère de plus en plus à l'idée d'une intervention plus soucieuse de la différenciation des situations et plus axée sur la réinsertion professionnelle, les avancées vers les mesures actives se font sans changer l'économie générale de l'investissement public par rapport à l'emploi. Barbier et Théret parlent d'une activation à la française «qui mêle des aspects de solidarité et de citoyenneté [...] à des aspects de réforme libérale du marché du travail ${ }^{36}{ }$. Ainsi, malgré la rhétorique des «trappes à pauvreté» dont nous avons souligné l'émergence, les éléments disciplinaires des politiques d'activation, sur les plans administratifs, et la dévalorisation des minima sociaux, qui sont présents dans la démarche du workfare à la britannique ou à l'américaine, n'arrivent pas à s'imposer ${ }^{37}$. Mais cette politique d'incitation au retour au travail par l'activation de la main-d'œuvre, si elle fait davantage écho à la social-démocratie, n'en a ni les

35. Stéphanie Jamet, Lutter contre la pauvreté et l'exclusion sociale en France, op. cit.

36. Jean-Claude Barbier, Bruno Théret, Le nouveau système français de protection sociale, op. cit., p. 97.

37. Bruno Palier, Gouverner la sécurité sociale: Les Réformes du système français de protection sociale depuis 1945, op. cit. 
ressources, ni le volet décisif de formation et reclassification, ni la logique de droits universels extensifs ${ }^{38}$.

Le passage du RMI (1988) au RSA (2007) témoigne bien de ces ambiguïtés. Le Revenu minimum d'insertion fut conçu pour assurer un revenu minimum aux sans-emploi de longue durée, qui n'étaient pas admissibles aux mesures du filet de sécurité et défiaient donc, par leur seule existence, la protection citoyenne de l'État social français. Mais il incarnait également un droit à un minimum social pensé en termes d'insertion à la vie sociale et professionnelle. Cependant, l'horizon de la réinsertion prenait le visage d'un contrat moral, non d'une obligation encadrée par des dispositifs punitifs à l'anglo-saxonne. En 2004, on crée un Revenu minimum d'activité qui cherche à améliorer l'insertion sociale et professionnelle des prestataires, notamment par la personnalisation de l'aide ${ }^{39}$. De lutte à la pauvreté, on passe à l'insertion sur le marché du travail par l'activation. Ce Revenu minimum d'activité peut donner lieu à des sanctions, mais la mise en application de cette possibilité laisse place à interprétations divergentes ${ }^{40}$. En 2007, le Revenu de solidarité active remplace ces dispositifs et devient à la fois un complément de revenu pour les travailleurs pauvres et un revenu minimum pour ceux qui ne travaillent pas. Pour certains observateurs, le risque est d'institutionnaliser le statut de «travailleur précaire assisté», d'un «sous-salariat déguisé»: «Si l'on peut espérer que, pour certains, ce statut ne soit qu'un pis-aller temporaire avant d'accéder à un emploi stable non assisté,

38. Jean-Claude Barbier, Valeria Fargion, «Continental Inconsistencies on the Path to Activation: Consequences for Social Citizenship in Italy and France», European Societies, vol. 6, $\mathrm{n}^{\circ}$ 4, 2004.

39. Elie Alfandari, «Revenu minimum, insertion, activité: logique économique et/ou logique sociale?», Revue de droit sanitaire et social, $\mathrm{n}^{\circ}$ 1, 2004.

40. Robert Lafore, «La décentralisation du revenu minimum d'insertion», Revue de droit sanitaire et social, $\mathrm{n}^{\circ}$ 1, 2004, p. 20-21. 
on peut déjà craindre que le RSA participe à un mode généralisé de mise au travail des plus pauvres dans les segments les plus dégradés du marché de l'emploi ${ }^{41}$.» On touche ici les paradoxes des politiques d'insertion du modèle français et son revers: la création d'une pauvreté aux contours bien spécifiques.

\section{D'une assise égalitaire à la montée en force récente du précariat et des working rich}

La dualisation du marché du travail en France structure en profondeur la pauvreté. Les politiques d'activation ont des effets contrastés, et dans l'ensemble, l'aide publique et la sortie de certains mécanismes de solidarité ne semblent pas garantir des conditions de vie permettant d'échapper à la pauvreté. Il est remarquable qu'un tiers des bénéficiaires du RMI qui en sont sortis et qui ont repris un emploi déclarent n'avoir obtenu «aucune amélioration sur le plan financier [...] et que près de $12 \%$ constatent même une dégradation ${ }^{42} »$. Parallèlement, on constate une relation claire entre les contrats aidés et l'émergence de travailleurs pauvres ${ }^{43}$. Même du côté des travailleurs réguliers, il faut souligner que si le salaire minimum (65\% du salaire médian) est le plus élevé des pays de l'OCDE, il ne permet pas pour autant à un ménage avec des enfants et où l'une des personnes est au SMIC à plein temps d'échapper à la pauvreté. Or, c'est près de $14 \%$ de la population active qui est payée au SMIC en 2008. C'est le

41. Nicolas Duvoux, Serge Paugam, La régulation des pauvres, op. cit., p. 99-100.

42. Danièle Guillemot, Patrick Pétour, Hélène Zajdela, «Trappe à chômage ou trappe à pauvreté: Quel est le sort des allocations du RMI?», Revue économique, vol. 53, $\mathrm{n}^{\circ}$ 6, 2002, p. 1245.

43. Jean-Claude Barbier, Valeria Fargion, «Continental Inconsistencies on the Path to Activation: Consequences for Social Citizenship in Italy and France», op. cit. 
développement de ce précariat, comme le désigne Robert Castel, entre travail et chômage, assurance et assistance, qui est le produit de la configuration que nous avons analysé ${ }^{44}$. Selon cet auteur, on assiste à la dégradation de la solidarité: non plus cette interdépendance organique entre les membres de la société, partageant au-delà de leurs différences les mêmes droits, socles de la citoyenneté sociale, mais bien une inclinaison morale à venir en aide aux plus démunis. «La différence est que dans le premier cas de figure on est dans une société où tout le monde est citoyen à part entière, tandis que dans le second la population est clivée entre ceux qui assurent leur indépendance sociale par leur travail ou par leur patrimoine et une nébuleuse d'assistés en situation de dépendance parce qu'ils ne peuvent pas, à partir de leur travail, acquérir les conditions de cette indépendance, et qu'ils n'ont pas d'autres ressources pour y parvenir ${ }^{45}$.»

Si on mesure le basculement de perspective qu'induit la cristallisation de ce précariat, à la lumière de l'héritage solidariste républicain français, il faut souligner cependant que dans une perspective comparée, la pauvreté est moins importante en France que dans la moyenne des pays de l'Europe à 15 et ce, pour toutes les strates d'âge. Le taux de pauvreté des enfants est nettement sous la moyenne européenne, contrastant par exemple avec ce que nous avons vu en Grande-Bretagne. De plus, le taux de pauvreté, qu'on le calcule à $50 \%$ ou à $60 \%$ du revenu médian, baisse depuis les années 1970, même si l'on constate un plateau depuis les années 1990. Cette évolution est d'abord le fait d'une impor-

44. Robert Castel, L'insécurité sociale: Qu'est-ce qu'être protégé?, Seuil, 2003; Robert Castel, «Au-delà du salariat ou en deçà de l'emploi? L'institutionnalisation du précariat», La montée des incertitudes. Travail, protection, statut des individus, Seuil, 2009, p. 159-183.

45. Ibid., p. 183. 
tante contraction de la pauvreté chez les retraités, grâce aux dispositifs de retraites et de couvertures médicales.

Par contre, on peut rappeler la situation difficile de trois groupes au sein de la population (dont l'appartenance est non exclusive) à cause de leur situation sur le marché du travail. Les jeunes (moins de 25 ans) connaissent un fort taux de chômage, puisqu'il atteint 20\% des jeunes de 15 à 24 ans actifs, contre 9,O\% pour les 25-49 ans. Le deuxième groupe est lié aux familles avec un seul des parents à leur tête, qui représentent $7 \%$ des ménages, mais $20 \%$ des ménages pauvres $^{46}$. Ces ménages ont dans une très forte proportion à leur tête des femmes, qui cumulent les handicaps sur le marché de l'emploi français (notamment en termes de rémunération) par rapport à leurs homologues masculins. Enfin, le troisième groupe renvoie aux populations immigrées. Leur taux de pauvreté est plus élevé et il l'est d'autant plus que les personnes immigrées viennent de l'extérieur d'Europe. Ainsi, le taux de pauvreté d'un ménage d'origine du Maghreb est de $27,3 \%$ (avec un seuil de pauvreté à 50\% du revenu médian), alors qu'il est de 8,3\% pour un ménage d'origine européenne (mais non français): ces pourcentages sont à mettre en relation avec le taux pour la population non immigrée, taux qui est d'à peine $5,1 \%{ }^{47}$.

On ne peut qu'être frappé par la grande stabilité de l'inégalité des revenus en France au cours des dernières années. Tous les indicateurs illustrent bien le phénomène. L'indice Gini témoigne d'une inégalité des revenus relative modérée, plaçant la France entre la situation des pays d'économie

46. France. Le rapport de l'Observatoire national de la pauvreté et de l'exclusion sociale 2007-2008, Observatoire national de la pauvreté et de l'exclusion sociale et La Documentation française, 2008.

47. Stéphanie Jamet, Lutter contre la pauvreté et l'exclusion sociale en France, op. cit. 
TABLEAU 4.3

Indice de Gini pour l'inégalité des revenus, avant et après les transferts, 1996-2003

\begin{tabular}{|l|c|c|}
\hline & Avant transfert & Après transfert \\
\hline 1996 & 0,335 & 0,270 \\
\hline 1997 & 0,356 & 0,270 \\
\hline 1998 & 0,357 & 0,268 \\
\hline 1999 & 0,359 & 0,269 \\
\hline 2000 & 0,359 & 0,272 \\
\hline 2001 & 0,358 & 0,272 \\
\hline 2002 & 0,350 & 0,267 \\
\hline $2002^{\star}$ & 0,351 & 0,269 \\
\hline 2003 & 0,350 & 0,268 \\
\hline
\end{tabular}

Source: France. Rapport sur les stratégies pour la protection sociale et l'inclusion sociale 20062008, Paris, Ministère du Travail, des Relations sociales, de la Famille et de la Solidarité, 2006, annexes p. 17.

sociale de marché et des pays d'économie libérale de marché. L'intervention publique a un impact réel puisqu'il a contribué à un tassement de l'inégalité. Pour illustrer cela, considérons qu'en 2004, la redistribution opérée par l'impôt correspond à un transfert net d'environ $3 \%$ de l'ensemble des revenus des ménages des $20 \%$ les plus riches vers le reste de la population ${ }^{48}$. Un regard plus panoramique sur l'évolution des écarts de niveau de vie permet par contre de saisir, après une séquence de baisse entre 1970 et 1984, puis une très légère baisse de 1984 à 2004, une remontée importante de 2004 à $2006^{49}$.

Vue sous l'angle de la «distance économique», l'inégalité semble également contenue, car le rapport entre le décile des mieux nantis et des plus mal nantis, loin de s'accroître, se

48. Marion Navarro, «La distribution des revenus et des patrimoines en France», Regards croisés sur l'économie, vol. 2, nº 4, 2008, p. 51-53. 2009.

49. Liêm Hoang Ngoc, Sous la crise, la répartition des revenus, La dispute, 
tasse un peu. Contrairement à la Grande-Bretagne, où l'on observe un accroissement de l'inégalité par l'accentuation des extrêmes, ici nous avons plutôt un resserrement des différences et donc une moins grande «distance économique» entre les diverses catégories de revenu. Il est assez remarquable que le cinquième de la population au bas de l'échelle voie sa part des revenus légèrement augmenter, alors que le cinquième de la population drainant le plus de revenus voit sa situation rester à peu près inchangée durant la décennie 1996-2005. Cependant, derrière l'aspect rassurant de cet indicateur, un panorama par déciles permet de saisir la persistance d'une répartition de la richesse inégalitaire. Ainsi, les classes modestes (30\% de la population dont le niveau de vie est inférieur à 1000 euros par mois) détiennent $13,1 \%$ du revenu national. Les classes moyennes (4O\% de la population dont le niveau de vie se situe entre 1000 et 1600 euros par mois) vivent avec $42,5 \%$ du revenu national. Les classes aisées (20\% de la population avec un niveau de vie entre 1600 et 2300 euros par mois) détiennent $27,6 \%$ du revenu national. Enfin, les classes à hauts revenus (10\% de la population dont le niveau de vie est supérieur à 2300 euros par mois) se voient attribuer un quart du revenu national ${ }^{50}$. Surtout, si l'on s'intéresse maintenant aux 0,01\% des foyers les plus riches, on s'aperçoit que leur revenu réel a crû de 42,6\% de 1998 à 2005, contre 4,6\% pour les 90\% les moins riches $^{51}$. Après une période de grande stabilité de l'évolution des revenus réels de 1980 à la fin des années 1990, durant laquelle le revenu moyen comme les hauts revenus stagnaient, les seconds ont connu une explosion dans les années

50. Liêm Hoang Ngoc, Sous la crise, la répartition des revenus, op. cit.

51. Camille Landais, Les hauts revenus en France (1998-2006), une explosion des inégalités, School of Economics, juin 2007. www.inegalites.fr/spip.php? article705. 
GRAPHIQUE 4.3

Taux de croissance annuel moyen des revenus réels (hors plusvalues) pour différents fractiles, France/États-Unis, 1980-2005

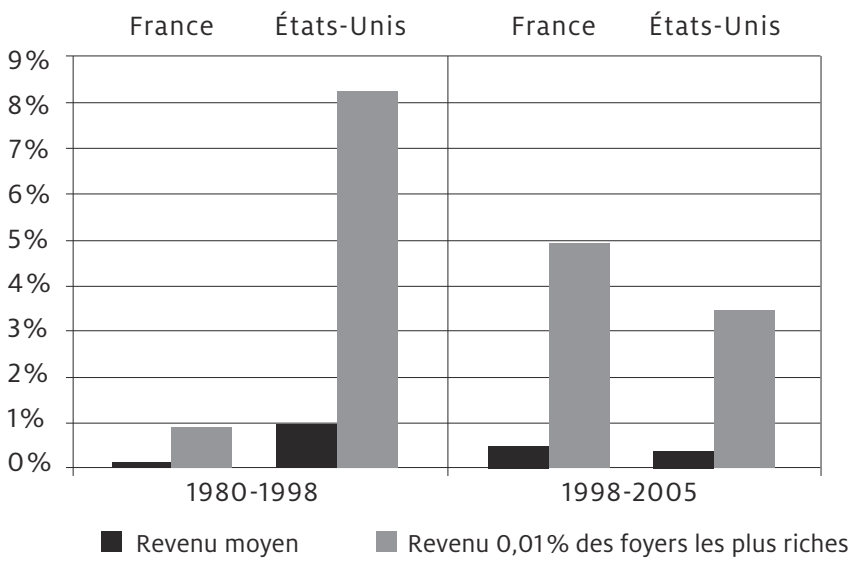

Source: Camille Landais, Les hauts revenus en France (1998-2006), une explosion des inégalités, School of Economics, juin 2007, p. 24.

1990, avec un taux de croissance même supérieur à celui observé aux États-Unis. Cette évolution sur les dernières années contraste avec l'évolution comparée de la part du revenu national captée par le $1 \%$ le plus riche des populations américaines, britanniques et françaises. Si ce centile percevait dans les trois cas près de $8 \%$ des revenus en 1983, la proportion est la même dans le cas français en 1999, mais elle a été multipliée par 1,5 au Royaume-Uni et a doublé aux États-Unis ${ }^{52}$.

52. Thomas Piketty, «Top Incomes Over the Twentieth Century: A Summary of Main Findings», Anthony Atkinson, Thomas Piketty (dir.), Top Incomes over the Twentieth Century: A Contrast Between Continental European and English-Speaking Countries, Oxford University Press, 2007. 
Parallèlement à l'explosion des plus hauts salaires, on observe également sur cette période la croissance des revenus du patrimoine, soumis à une fiscalité beaucoup moins redistributive que celle sur les revenus du travail. Comme le souligne Camille Landais, l'évolution de la fiscalité des revenus et du patrimoine a fortement baissé de 1998 à 2006 : baisse du taux marginal le plus élevé du barème de l'impôt sur le revenu (de $54 \%$ à $40 \%$ ), mise en place du bouclier fiscal à $50 \%$, forte baisse des droits de succession des hauts patrimoines $^{53}$. Sur ce dernier point, si les responsables politiques français tendent à revendiquer l'affiliation du modèle républicain français (par définition, toujours «unique» et «inclassable») à l'horizon social-démocrate, généreux et égalitaire, force est de constater que c'est davantage le modèle libéral qui semble donner le ton si l'on considère la répartition de la richesse.

53. Landais Camille, Les hauts revenus en France (1998-2006), une explosion des inégalités, op. cit. 



\section{La lutte québécoise contre la pauvreté dans un contexte inégalitaire}

Par rapport aux autres configurations sociales dont nous avons parlé, le cas du Québec se distingue par plusieurs aspects. Au sein de la diversité canadienne, c'est le Québec qui présente les différences de loin les plus importantes en termes d'intervention de l'État social ${ }^{1}$. C'est aussi le Québec qui présente les distances les plus grandes de toutes les provinces canadiennes par rapport aux États-Unis et au modèle libéral d'État social. C'est, la province qui a, a priori, le plus d'affinité avec les modèles européens ${ }^{2}$. Ce positionnement, dans l'entre-deux, se traduit par un niveau de dépenses publiques plus élevé qu'ailleurs au Canada, des charges sociales relativement importantes et un niveau d'emploi public qui atteste d'une forte présence étatique.

Cette caractéristique se comprend dans sa perspective historique. La construction d'un État social fort est liée à la volonté d'affirmation nationale qui nécessite un État capable

1. Paul Bernard, Sébastien St-Arnaud, «Du pareil au même? La position des quatre principales provinces canadiennes dans l'univers des régimes providentiels», Canadian Journal of Sociology/Cahiers canadiens de sociologie, vol. 29, $\mathrm{n}^{\circ}$ 2, 2004, p. 209-236.

2. Idem. 
d'initiatives et d'innovations ${ }^{3}$. Par ailleurs, au Canada, l'intervention publique en matière sociale implique les deux gouvernements (fédéral et provincial). Certaines compétences sont partagées (emploi, famille, logement), d'autres non (éducation, santé). Le Québec est responsable de la gestion des programmes d'assistance sociale, une politique d'aide de dernier recours pour les personnes sans emploi, des programmes d'activation qui les accompagnent et de certaines mesures en direction des familles (soutien du revenu et services), alors que le gouvernement fédéral gère le régime d'assurance-chômage (qui se nomme assurance-emploi), ainsi que des programmes de soutien du revenu en direction des familles ${ }^{4}$. Les programmes de formation professionnelle sont gérés au sein du programme provincial d'assistance social, ce qui limite singulièrement leur portée ${ }^{5}$.

Outre ce partage constitutionnel des champs de compétences des gouvernements, certaines normes codifient l'utilisation que le gouvernement provincial peut faire des transferts fédéraux. Par exemple, l'entente Canada-Québec relative au marché du travail impose des restrictions quant à l'utilisation des sommes qui proviennent du Compte d'assurance-emploi ${ }^{6}$. Autrement dit, le gouvernement du Québec et les Québécois détiennent une marge de manœuvre importante pour décider du sort collectif des personnes les plus

3. Alain Noël, «Construire la souveraineté par la base», Yvon Leclerc, Claude Béland (dir.), La voie citoyenne: pour renouveler le modèle québécois, Plurimédia, 2003, p. 255-273.

4. Michel Laffut, Marie-Renée Roy, Pauvreté et exclusion sociale: partage d'expériences entre Wallonie et Québec, De Boeck, 2007, p. 20.

5. Sylvie Morel, Modèle du workfare ou modèle de l'insertion? La transformation de l'assistance sociale au Canada et au Québec, Condition féminine Canada, dsp-psd.tpsgc.gc.ca/Collection.

6. Gouvernement du Québec, Comité consultatif de lutte contre la pauvreté et l'exclusion sociale. Planification et orientations 2006-2009, 2007. 
démunies de leur société, mais ils appartiennent à l'ensemble fédéral canadien et ses contraintes.

Comme en Grande-Bretagne, le travailleur pauvre est une figure courante au sein des personnes pauvres au Québec; mais à la différence du gouvernement britannique, les gouvernements québécois successifs ont investi activement le champ de la lutte contre la pauvreté et l'exclusion sociale, contribuant à modifier les représentations sociales traditionnelles qui y étaient liées. Pour en arriver là, et en quelque sorte emprunter une voie hors des sentiers battus des régimes libéraux d'État social, la société québécoise a progressivement construit des pratiques d'intervention publique qui font une place croissante aux acteurs sociaux dans les processus politiques.

Cette société laisse tout de même $10 \%$ de sa population en bas de l'échelle. Comme dans les autres cas analysés, les personnes démunies ont souvent un sexe (les femmes) et une origine (les personnes d'origine immigrante). Plus inclusif que son voisin du Sud et que la plupart des provinces canadiennes, le Québec fait face à des défis semblables.

\section{Une situation fragile}

Entre 1997 et 2005, les Canadiens et les Québécois ont bénéficié d'un taux d'emploi élevé et d'une croissance économique soutenue. L'inflation a été bien maîtrisée tout au long de la période (elle est de 1,6\% en 2007); le taux de chômage est en baisse, passant de $11,4 \%$ à 7,2\% en $2007^{7}$. Par ailleurs, un effort collectif important est consenti en éducation et en santé.

7. Institut de la statistique du Québec, Taux de chômage selon le groupe d'âge et selon le sexe, moyennes annuelles, Québec, 1976 à 2007, www.stat.gouv. qc.ca/donstat. 
Par exemple, la province consacre au secteur de l'éducation un peu plus que le Canada (2 119 \$ US PPA), ce qui se traduit par une part des dépenses d'éducation dans le PIB plus considérable ${ }^{8}$.

Cette embellie économique, qui fait suite à plusieurs années difficiles, n'a toutefois pas profité à tous de manière équivalente. En même temps que certains travailleurs obtenaient des augmentations de salaire (en particulier dans les secteurs d'activité suivants: extraction minière et extraction de pétrole et de gaz, commerce de gros, finances et assurances, soins de santé et assistance sociale ${ }^{9}$ ), d'autres connaissaient une précarisation de leurs conditions de travail, le nombre d'emplois atypiques offrant peu ou pas d'avantages sociaux (travail sur appel, à contrat, à temps partiel) augmentant sensiblement.

Au chapitre de la pauvreté, la situation est paradoxale. La plupart des indicateurs statistiques montrent que la pauvreté mesurée de façon absolue a diminué durant ces années de croissance économique, passant de $12 \%$ en 1996 à $5 \%$ en $2005^{10}$ au Canada. La situation économique enviable s'est aussi traduite par une embellie du côté du taux des personnes à faible revenu au Canada, le pourcentage passant de 15,7\% de la population à faible revenu après impôt en 1996 à 10,8\% en 2006. Mais, ce qui est plus remarquable encore, c'est l'évolution que le Québec a connue durant cette période.

8. Michel Laffut, Marie-Renée Roy, Pauvreté et exclusion sociale. Partage d'expériences entre Wallonie et Québec, op. cit.

9. Statistique Canada, Évolution de la rémunération horaire moyenne des salariés rémunérés à l'heure selon la branche d'activité, 2003-2007, www40. statcan.ca/.

10. Chris Sarlo, What is Poverty? Providing Clarity for Canada, Fraser Institute, 2008. 
TABLEAU 5.1

Personnes à faible revenu après impôt, en proportion de la population totale, 1996-2005

\begin{tabular}{|l|c|c|c|}
\hline & 1996 & 2000 & 2005 \\
\hline Canada & 15,7 & 12,5 & 10,8 \\
\hline Ontario & 14,2 & 10,8 & 10,3 \\
\hline Québec & 18,8 & 14,8 & 11,3 \\
\hline
\end{tabular}

Source: Statistique Canada, Le revenu au Canada 2005, Ottawa, mai 2007, p. 88, 89 et 100, cité par Alain Noël, «Gouverner à plusieurs. La protection sociale au Canada et au Québec », Informations sociales, vol. 143, 2007, p. 25.

\section{TABLEAU 5.2}

Familles comptant des personnes de moins de 18 ans à faible revenu après impôt, en proportion de la population totale, 1996-2005

\begin{tabular}{|l|c|c|c|}
\hline & 1996 & 2000 & 2005 \\
\hline Canada & 18,6 & 13,8 & 11,7 \\
\hline Ontario & 18,0 & 12,8 & 12,6 \\
\hline Québec & 20,1 & 16,0 & 9,6 \\
\hline
\end{tabular}

Source: Statistique Canada, Le revenu au Canada 2005, Ottawa, mai 2007, p. 88, 89 et 100, cité par Alain Noël, «Gouverner à plusieurs. La protection sociale au Canada et au Québec », Informations sociales, vol. 143, 2007, p. 25.

Au total, entre 1996 et 2005, le Québec a réussi à rejoindre la moyenne canadienne et l'Ontario au chapitre du taux de faible revenu, mais il a également fourni des efforts supplémentaires entre 2000 et 2005 . Comme le souligne Alain Noël ${ }^{11}$, cette évolution est encore plus claire si on considère la situation des familles avec enfants de moins de 18 ans. Le Québec a dépassé à ce sujet la moyenne canadienne et ontarienne.

Cependant, le Québec n'est pas aussi prospère que le reste du pays. Par exemple, le PIB au prix du marché par habitant

11. Statistique Canada, Le revenu au Canada 2005, Ottawa, mai 2007, p. 88, 89 et 100, cité par Alain Noël, «Gouverner à plusieurs. La protection sociale au Canada et au Québec», Informations sociales, 143, 2007. 
en 2007 y est de 6739 \$ de moins qu'en Ontario, de 4707 \$ de moins qu'en Colombie-Britannique et de 36 oog $\$$ de moins qu'en Alberta ${ }^{12}$. Depuis 2004, le ralentissement économique est plus sensible au Québec que dans ces autres provinces. Il en va de même pour les indicateurs relatifs à l'emploi. En 2007, le taux d'emploi au Québec est plus faible que dans le reste du Canada (respectivement 71,6\% et 73,6\%, dans la population des 15-64 ans) et le taux de chômage est plus élevé $(7,2 \% \text { et } 6,0 \% \text { respectivement })^{13}$. Une situation fragile, qui peut rapidement basculer.

Au-delà de la question de la «quantité» de pauvreté, il est nécessaire de considérer celle de l'aggravation de l'incidence de la pauvreté. Si la proportion de personnes pauvres en chiffre absolu a diminué, celles qui sont en situation de pauvreté sont plus pauvres qu'avant et, surtout, c'est l'écart entre les riches et les pauvres qui s'est accru ${ }^{14}$. Au Québec, comme au Canada, l'inégalité du revenu familial après impôt croît très fortement depuis les années 1990, en partie à cause d'une accélération de l'inégalité par la distribution primaire des revenus par le marché.

La croissance de l'inégalité a été telle, que même avec un effet de redistribution très significatif, l'indice d'inégalité en 2004 est le plus élevé depuis au moins 30 ans ${ }^{15}$. Ainsi, la polarisation des revenus devient de plus en plus grande. En

12. Institut de la statistique du Québec, PIB aux prix du marché par habitant, 20O2-2O11, www.stat.gouv.qc.ca/donstat/.

13. Institut de la statistique du Québec, Taux de chômage selon le groupe d'âge et selon le sexe, moyennes annuelles, Québec, 1976 à 2007, www.stat.gouv. qc.ca/donstat/.

14. Institut de la statistique du Québec, Coefficient de Gini calculé selon les concepts de revenu différents, Québec, 1996 à 2007, www.stat.gouv.qc.ca/ donstat/.

15. Institut de la statistique du Québec, Coefficient de Gini et contributions des composantes de revenu, toutes les familles, Québec, 1979-2004, www.stat.gouv. qc.ca/publications/. 
GRAPHIQUE 5.1

Indices d'inégalité du revenu après impôt - Coefficient de Gini, 1976 à 2004

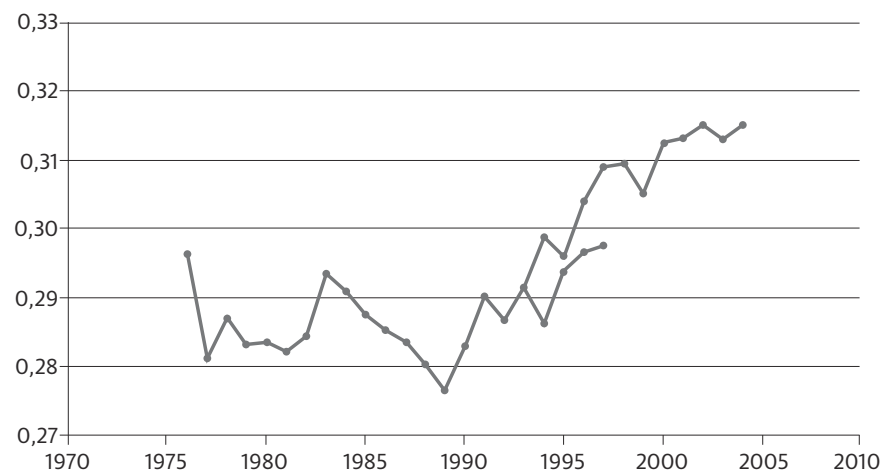

Source: Andrew Heisz, Inégalité et redistribution du revenu au Canada: de 1976-2004, Ottawa, Statistiques Canada, 2007, p. 42.

bref, si la société québécoise est collectivement moins pauvre aujourd'hui, «l'ascenseur social» ${ }^{16}$ ne fonctionne pas à la même vitesse et selon les mêmes principes pour tous.

Pour comprendre cette situation paradoxale, à la fois en nette progression et peu égalitaire, nous revenons, dans un premier temps, sur les transformations du marché du travail, qui sont une des composantes centrales de la pauvreté au Québec.

\section{Un marché du travail fragilisé}

L'économie québécoise a enregistré une croissance économique annuelle moyenne de 2,7\% entre 1997 et 2004, ce qui

16. Pour reprendre l'expression du Collectif pour un Québec sans pauvreté. 
a permis une création d'emplois à un taux annuel de $2,1 \%{ }^{17}$. Le taux de chômage est passé de 9,4\% à $7 \%$ en 2007. Au total, en 2007, le taux d'emploi était de 61,0\%. Cependant, le marché du travail est de plus en plus fragilisé18 ${ }^{18}$ Par exemple, la probabilité pour une jeune adulte d'occuper un emploi syndiqué ou couvert par une convention collective a nettement diminué (6/10 pour la cohorte née dans les années 1940 et 4/10 pour la cohorte née dans les années 1970) ${ }^{19}$. Or, le niveau de revenu est directement lié à la nature de l'emploi, les emplois atypiques étant plus souvent «mal payés» et sans protection pour le travailleur que les emplois à temps plein à durée indéterminée. Il y a un lien direct, mais souvent occulté, entre la nature des emplois et les questions de pauvreté et d'exclusion sociale.

La déréglementation du marché du travail a entraîné une stagnation des salaires moyens ${ }^{20}$, une baisse ou au moins une stagnation du salaire minimum (en tenant compte de l'indice des prix à la consommation) ${ }^{21}$ et une croissance de l'insécurité en emploi.

L'existence même de «travailleurs pauvres» contredit le discours selon lequel l'emploi est le principal mécanisme de redistribution sociale et elle infirme surtout la croyance que,

17. Institut de la statistique du Québec, État du marché du travail au Québec. Bilan de l'année, 2008.

18. Ron Saunders, Risques et possibilités: à la recherche d'options pour les travailleurs vulnérables, Canadian Policy Research Networks, 2006.

19. Charles Fleury, «La génération $X$ au Québec: une génération sacrifiée?», Recherches sociographiques, vol. 49, n 3, 2008, p. 475-499.

2O. Institut de statistique du Québec, Rémunération horaire moyenne des employés selon diverses caractéristiques, moyennes annuelles, Québec, 1997 à 2007, www.stat.gouv.qc.ca/donstat/.

21. En mai 2007, le salaire minimum a été augmenté de $0,25 \$$, passant à 8 \$/h; en mai 2008, il a connu une hausse encore plus importante de 0,50 \$. Plus élevé qu'aux États-Unis et qu'en Ontario, le salaire minimum québécois réduit l'incidence de la pauvreté. Institut de la statistique du Québec, Taux de salaire minimum sur une base horaire pour les travailleurs adultes, www.stat. gouv.qc.ca/donstat/. 
dans une société basée sur l'économie de marché, la pauvreté ne peut résulter que de l'absence de travail ${ }^{22}$. Les conséquences de l'existence d'une catégorie de personnes «pauvres qui travaillent» vont bien au-delà des questions de salaire et de revenu. C'est une question d'accès à la pleine citoyenneté et de reconnaissance de la contribution des personnes à la vie collective ${ }^{23}$.

Le phénomène des travailleurs pauvres résulte d'un ensemble de décisions politiques et économiques prises au cours des vingt dernières années, qui avaient pour principal objectif de garantir au marché du travail une flexibilité croissante. La déréglementation du marché du travail est un phénomène généralisé dans les économies des pays industrialisés depuis le début des années 1980. Cependant, elle a été plus soutenue en Amérique du Nord qu'en Europe continentale (France). Elle a également été appliquée avec plus de violence qu'en Europe du Nord (Danemark), où flexibilité ne rime pas toujours avec insécurité pour les travailleurs. C'est pourquoi le phénomène des travailleurs pauvres est plus marqué au Québec et en Grande-Bretagne, où l'assouplissement des règles régissant l'emploi s'est directement traduit par un effritement des droits des travailleurs.

Le phénomène des travailleurs pauvres est complexe. Il comporte, cependant, trois dimensions centrales: l'insuffisance de la rémunération du travail (salaire minimum trop faible); l'insuffisance de la quantité de travail (temps partiel, travail à contrat, à la pige, etc.); l'insuffisance de la protection sociale liée aux statuts (travailleurs ou chômeurs) ou aux changements de statuts. Au Canada, le premier cri d'alarme

22. Pierre Joseph Ulysse, Travail et pauvreté. Quand le travail n'empêche plus d'être pauvre!, Forum régional de développement social de l'île de Montréal, 2006, p. 5-8.

23. Barbara Ehrenreich, L’Amérique pauvre, Éditions 10/18, 2004. 
a été lancé par le Conseil national du bien-être (CNBE) qui, dans un rapport daté de 1977, maintient qu'une large proportion des personnes pauvres au Canada sont en fait des employés ou des personnes qui travaillent ${ }^{24}$. Dans la première moitié des années 1990, la proportion d'emplois non standards (c'est-à-dire tous les emplois différents de l'emploi salarié à temps plein permanent) a considérablement progressé puis s'est stabilisée ${ }^{25}$. Cependant, l'insécurité liée à ces emplois a continué de progresser.

Statistiquement, «un travailleur à faible revenu» est un individu démontrant un effort de travail considérable durant toute l'année (plus de 910 heures), mais dont le revenu familial se situe sous le seuil de faible revenu ${ }^{26}$. Selon cette définition, Roy, Fréchet et Savard ${ }^{27}$ ont déterminé que les travailleurs à faible revenu représentent $31 \%$ de toutes les personnes à faible revenu familial. Par ailleurs, selon une étude menée par le Québec en 2005, les travailleurs indépendants à temps plein travaillant toute l'année (42\%) sont plus susceptibles d'être à faible revenu que les employés se trouvant dans les mêmes conditions (11\%). Le faible revenu est plus fréquent chez les femmes travaillant à temps plein toute l'année (16\%) que chez les hommes (7\%). Les personnes n'ayant pas de diplôme, les immigrants récents, les personnes seules, les mères de familles monoparentales représentent $37 \%$ de tous les travailleurs à temps plein, mais $71 \%$ de tous les travailleurs

24. Pierre Joseph Ulysse, Travail et pauvreté. Quand le travail n'empêche plus d'être pauvre!, op. cit.

25. Leah F. Vosko, Nancy Zukewich, Cynthia Cranford, «Precarious jobs: A new typology of employment», Perspectives on Labour and Income, vol. $4, \mathrm{n}^{\circ} 10,2003$, p. 1-11.

26. Institut de la statistique du Québec, Inventaire des indicateurs de pauvreté et d'exclusion sociale, Institut de la statistique du Québec et Ministère de l'Emploi et de la Solidarité, 2005.

27. Marie-Renée Roy, Guy Fréchet, Frédéric Savard, «Travail et pauvreté au Canada», Options politiques, IRPP, 2008. 
occupant des emplois rémunérés et à faible revenu ${ }^{28}$. Au final, les travailleurs pauvres québécois sont surtout des femmes, des jeunes immigrants et des Montréalais appartenant aux minorités visibles. Parmi ces derniers, les personnes de couleur constituent le groupe des travailleurs les plus pauvres ${ }^{29}$.

Dans ce contexte, le gouvernement fédéral et celui du Québec ont agi sur deux fronts: les soins palliatifs pour soulager les situations les plus criantes de pauvreté; des actions plus ciblées en direction de certaines catégories de personnes (enfants, travailleurs à faible revenu avec famille) et le développement d'une vision de long terme devant réduire radicalement la pauvreté. Dans la partie suivante, nous présentons les grandes lignes de l'intervention publique au regard de son impact sur les questions de pauvreté et d'exclusion sociale.

\section{Des mesures et dispositifs de protection sociale qui soulagent}

L'intervention publique a joué un rôle positif et important eu égard à la diminution substantielle de la pauvreté au Québec $^{30}$. Les gouvernements successifs se sont en effet engagés dans une série de programmes sociaux depuis la fin des années 1990 qui avaient explicitement pour but de réduire le nombre et l'incidence de la pauvreté.

28. Richard P. Chaykowski, Non-standard Work and Economic Vulnerability: Defining Vulnerability in the Labour Market, Réseaux canadiens de recherche en politiques publiques, 2005; René Morissette, Garnett Picot, Le travail peu rémunéré et les familles économiquement vulnérables depuis 20 ans, Rapport de recherche de la Direction des études analytiques, Statistique Canada, 2005.

29. Pierre Joseph Ulysse, Travail et pauvreté. Quand le travail n'empêche plus d'être pauvre!, op. cit.

30. Marie-Renée Roy, Guy Fréchet, Frédéric Savard, «Travail et pauvreté au Canada», op. cit. 
Comme nous l'avons mentionné, le gouvernement fédéral gère le programme d'assurance-emploi. La refonte de ce programme en 1997 a eu une incidence immédiate sur les provinces et le traitement politique de la pauvreté parce qu'elle a modifié les critères d'admissibilité au programme, excluant une part considérable des chômeurs de la couverture d'assurance ${ }^{31}$. Avec ces nouvelles règles, en 2007, seul un chômeur sur deux était admissible au programme ${ }^{32}$. Par un effet quasi mécanique, cette diminution du taux de couverture a directement influencé le nombre de personnes prestataires des programmes provinciaux d'assistance.

Historiquement, les programmes d'assistance servaient d'aide de dernier recours et s'adressaient surtout aux personnes éloignées ou assez éloignées du marché du travail. Aujourd'hui, le nombre de prestataires de l'aide sociale est fortement lié à la conjoncture économique. Une amélioration de la situation du marché du travail se traduit par une contraction du nombre de prestataires, beaucoup de ces prestataires étant des chômeurs et non des personnes ayant de la difficulté à exercer un emploi. D'ailleurs, sur l'ensemble des provinces du Canada, le pourcentage de la population bénéficiaire de l'aide sociale suit plus ou moins les mêmes cycles, rappelant les cycles économiques de l'économie canadienne. Avec les réformes du système de sécurité sociale canadien au milieu des années 1990, et les nouvelles responsabilités sociales qui incombaient aux provinces par ricochet, les gouvernements provinciaux se sont, eux aussi, penchés sur des restructurations de leurs programmes, modifiant parfois substantiellement le traitement politique

31. Canada, Service Canada, Loi sur l'assurance-emploi, Partie I, Prestations de chômage, www.servicecanada.gc.ca/.

32. Statistique Canada, Bénéficiaires d'assurance-emploi selon la province, www.statcan.ca/. 
GRAPHIQUE 5.2

Part relative des bénéficiaires d'aide sociale de la population et taux de chômage, 1968-2003

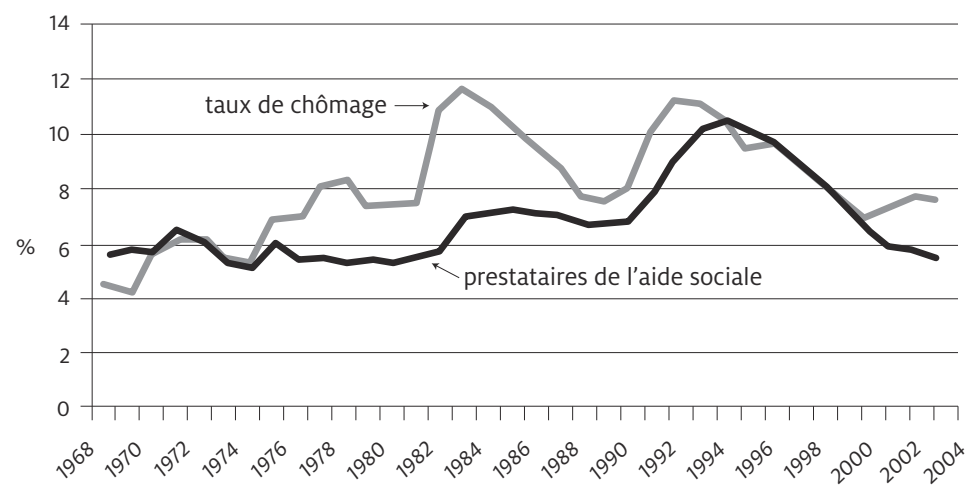

Source: Ken Battle, Michael Mendelson, Sherri Torjman, Towards a New Architecture for Canada's Adult Benefits, Caledon Institute of Social Policy, 2006, p. 5.

de la pauvreté. De fortes variations existent entre les provinces, qui ont été documentées et analysées ${ }^{33}$.

$\mathrm{Au}$ Québec, les réponses à ce changement structurel d'envergure ont été de plusieurs types: dans un premier temps, restructuration du programme d'assistance sociale, accroissement de la sévérité des mesures envers les personnes en chômage aptes au travail, mais aussi, depuis la fin des années 1990, mise en place d'une politique globale de lutte contre la pauvreté.

33. Paul Bernard, Sébastien St-Arnaud, «Du pareil au même? La position des quatre principales provinces canadiennes dans l'univers des régimes providentiels», Canadian Journal of Sociology/Cahiers canadiens de sociologie, vol. 29, $\mathrm{n}^{\circ}$ 2, 2004, p. 209-236; Gerard Boychuk, Patchwork of Purposes: The Development of Provincial Social Assistance Regimes in Canada, McGill-Queen's University Press, 1998. 


\section{Une société mobilisée par des enjeux de pauvreté}

Reconnaissant que la pauvreté mine le potentiel collectif d'une société et coûte cher en programmes sociaux, le Québec s'est fixé des objectifs ambitieux qui ont été partiellement atteints. Ces coûts sont directs - par exemple, le ministère de l'Emploi et de la Solidarité sociale (MESS), le principal ministère responsable de la lutte contre la pauvreté, avait un budget de 4 milliards de dollars en $2007^{34}-$, mais les coûts indirects sont également de plus en plus mentionnés (dépenses de soins de santé; lutte contre le décrochage scolaire, etc. $)^{35}$.

Cette vision globale de la pauvreté et de l'exclusion sociale qui semble prévaloir aujourd'hui au Québec ${ }^{36}$ est le fruit d'un travail de mobilisation effectué au départ au sein des groupes de la société civile autour de l'enjeu de «pauvreté zéro», aujourd'hui devenu celui d'un «Québec sans pauvreté ${ }^{37} »$. L'action des groupes et des coalitions a ensuite été relayée par le gouvernement qui en 2002 a adopté une loi-cadre contre la pauvreté et l'exclusion sociale, une première en Amérique du Nord. Lors de son adoption, l'objectif général de la loi était de mettre en mouvement l'ensemble des acteurs du Québec (gouvernement, partenaires socioéconomiques, collectivités régionales et locales, organismes

34. Pour l'année 2006-2007, les crédits du ministère de l'Emploi et de la Solidarité sociale s'élevaient à 4 033,3 millions de dollars. Gouvernement du Québec, Crédits et effectifs 2006-2007 du ministère de l'Emploi et de la Solidarité sociale, Emploi et Solidarité sociale, www.mess.gouv.qc.ca/ministere/creditseffectifs/.

35. Michel Laffut, Marie-Renée Roy, Pauvreté et exclusion sociale. Partages d'expériences entre Wallonie et Québec, De Boeck, 2007.

36. Alain Noël, «Loi visant à lutter contre la pauvreté et l'exclusion sociale du Québec», Perceptions, vol. 29, n ${ }^{\text {os }}$ 3-4, 2008, p. 16-22.

37. Pascale Dufour, «L'adoption de la Loi 112 au Québec: le produit d'une mobilisation ou une simple question de conjoncture politique?», Politique et Sociétés, vol. 23, nº 2-3, 2005, p. 159-182. 
communautaires) pour non seulement diminuer substantiellement la pauvreté à long terme, mais aussi (et surtout, comme nous le verrons plus loin) veiller à ne pas l'aggraver. Cette loi-cadre a été suivie de l'adoption d'un plan d'action gouvernemental en matière de lutte contre la pauvreté et l'exclusion sociale, Concilier liberté et justice sociale: un défi pour l'avenir (2004), et une réforme substantielle des programmes sociaux touchant directement les personnes en situation de pauvreté (2007 et 2008).

L'objectif premier de la loi est de guider le gouvernement et l'ensemble de la société québécoise dans la planification et la réalisation de stratégies et d'actions pour combattre la pauvreté, prévenir ses causes, en atténuer les effets sur les individus et les familles, contrer l'exclusion sociale et tendre vers un Québec sans pauvreté (art. 1). Le gouvernement s'était même fixé l'objectif de faire figurer le Québec, au bout de dix ans, parmi les nations industrialisées comptant le moins de personnes pauvres ${ }^{38}$.

La démarche du Québec se situait dans un mouvement international, la France ayant également adopté une loi semblable à la même période. Comme la France, le Québec inscrivait la lutte contre la pauvreté et l'exclusion sociale de manière formelle et durable dans le temps. Mais il modifiait aussi, et de manière substantielle, l'acception officielle de la pauvreté. Selon le préambule de la loi, la pauvreté et l'exclusion sociale peuvent porter atteinte aux droits et libertés des personnes ainsi qu'à leur dignité, qui sont des fondements de la justice et de la paix. Lutter contre la pauvreté et l'exclusion sociale devient alors un impératif national qui concerne

38. Québec, Assemblée nationale, Loi visant à lutter contre la pauvreté et l'exclusion sociale, $36^{\mathrm{e}}$ législature, deuxième session, Projet de loi $\mathrm{n}^{\mathrm{O}} 112$, Chapitre 2, «Stratégie nationale de lutte contre la pauvreté et l'exclusion sociale», article 4, 2002. 
tous les acteurs impliqués, au premier rang desquels se trouvent les personnes en situation de pauvreté.

Loin d'une définition quantitative de la pauvreté, la loi la définit comme étant «la condition dans laquelle se trouve un être humain qui est privé des ressources, des moyens, des choix et du pouvoir nécessaires pour acquérir et maintenir son autonomie économique et pour favoriser son intégration et son inclusion active dans la société québécoise» (art. 2). À l'égard des personnes et familles en situation de pauvreté, la loi prévoit de promouvoir le respect et la protection de leur dignité et de lutter contre les préjugés à leur égard, d'améliorer leur situation économique et sociale, de réduire les inégalités qui peuvent nuire à la cohésion sociale, de favoriser leur participation à la vie collective et au développement de la société. Enfin, la loi vise à développer et renforcer le sentiment de solidarité dans l'ensemble de la société québécoise afin de lutter collectivement contre la pauvreté et l'exclusion sociale.

La philosophie générale qui se dégage de la loi marque un tournant dans l'histoire des politiques sociales au Québec. En premier lieu, la loi rompt avec la logique comptable qui fait de la pauvreté une question de seuil. Celle-ci devient une question sociale à part entière qui interpelle la collectivité dans son ensemble et qui questionne l'accès à la citoyenneté, et donc aux droits des personnes démunies. Deuxièmement, elle dépasse une approche technicienne de la pauvreté qui voit dans la mise en place des mesures d'emploi et d'activation la planche de salut des personnes en situation de pauvreté. Elle propose, à l'inverse, de considérer la pauvreté sous ses multiples facettes (logement, revenu, emploi, santé) et prévoit une gamme intégrée d'actions pour prévenir et soutenir les personnes dans leurs parcours de vie. Finalement, la loi rompt, et là de manière radicale, avec l'approche puni- 
tive courante en Amérique du Nord, qui fait des personnes pauvres les seules responsables de leur sort et qui pose $a$ priori leur propension à choisir de ne pas travailler, de ne pas s'insérer, bref, d'être pauvres.

L'adoption de la Loi 112 a soulevé beaucoup d'espoir, tant du côté des acteurs sociaux, que du côté des fonctionnaires aux prises avec la gestion des programmes ${ }^{39}$.

Selon l'article 13 de la loi, un plan d'action devait être adopté et publié dans les 60 jours de la mise en vigueur de la loi, soit avant le 5 mai 2003. Celui-ci a finalement été rendu public près de 11 mois plus tard, le 2 avril 2004. Le délai de publication s'explique à la fois par le changement de gouvernement survenu en 2003, mais également par les batailles politiques auxquelles la production du plan a donné lieu ${ }^{40}$.

Selon l'article 14 de la loi, ce plan d'action devait fixer des cibles à atteindre, notamment pour améliorer le revenu des prestataires d'aide sociale et des travailleuses et travailleurs en situation de pauvreté. Le plan d'action devait également modifier le programme d'assistance-emploi et abolir les sanctions financières appliquées en cas de partage de logement, introduire le principe d'une prestation minimale non sujette à des réductions, améliorer le niveau permis de possession de biens et d'avoirs liquides, et enfin, exclure du calcul de la prestation pour toute famille ayant un enfant à charge un montant des revenus de pension alimentaire.

39. Colloque sur la loi 112 du Collectif pour un Québec sans pauvreté, L'horizon d'un Québec sans pauvreté vs la Loi visant à lutter contre la pauvreté et l'exclusion sociale: un processus et ses questions, Montréal, 2005.

40. FCPASQ, Lutte à la pauvreté. Bilan des 100 premiers jours du gouvernement Charest, Conférence de Jean-Yves Desgagnés, 2003; Mélanie Michaud, France Gagnon, Joël Gauthier, Le Plan d'action gouvernemental en matière de lutte contre la pauvreté et l'exclusion sociale. Étude de cas - Résumé, Groupe d'étude sur les politiques publiques et la santé, 2006; Alain Noël, Priorité au soutien du revenu: La mise en œuvre de la Loi québécoise visant à lutter contre la pauvreté et l'exclusion sociale, Canadian Policy Research Networks, 2004. 
Le plan d'action qui a finalement été adopté prévoit un déploiement de mesures sur cinq ans, fondé sur deux principes: 1) l'emploi est la première solution pour assurer la sécurité économique et l'inclusion sociale des personnes aptes au travail; et 2) une protection accrue doit être accordée aux personnes qui sont victimes d'incapacités ne leur permettant pas d'accéder à l'emploi ${ }^{41}$. D'emblée, on peut souligner que cette centralité de l'emploi dans la lutte contre la pauvreté et l'exclusion réduit la portée de la Loi 112, qui faisait de la pauvreté une question plus large que celle de l'accès à l'emploi.

Néanmoins, afin de reconnaître le principe de prestation minimale prévu par la loi, le plan abolit, dès avril 2005, les mesures coercitives et les pénalités financières pour refus de mesures ou d'emploi. La prestation de base ne peut donc plus faire l'objet d'une réduction, sauf en cas de fraude. Il ne s'agit pas encore d'un barème plancher, qui supposerait la couverture des besoins essentiels (ce que le montant de la prestation de base ne permet pas), mais il s'agit d'un tournant majeur dans l'histoire du traitement politique des personnes prestataires de l'aide sociale. En effet, dans les régimes libéraux de bien-être, dont fait partie le Québec, la logique punitive vis-à-vis des personnes sans emploi a fortement teinté les mesures prises à leur égard, les personnes sans emploi mais en mesure de travailler étant considérées avant tout comme responsables de leur situation et devant faire la preuve de leur volonté «à s'en sortir».

De plus, le gouvernement a investi plus de 2,5 milliards de dollars sur cinq ans autant dans des mesures visant à agir en direction des travailleurs à faible revenu et à valoriser 
le revenu de travail (mesure comme la «prime au travail», prime de participation à des mesures pour les prestataires de l'aide sociale, hausse régulière du salaire minimum) que dans le «soutien aux enfants» (un programme qui remplaçait des programmes existants tout en bonifiant substantiellement l'aide pour les familles à faible revenu) et le «logement social $^{42}{ }^{2}$. L'ensemble de ces mesures a joué comme facteur de redistribution au sein de la société québécoise.

Cependant, le plan ne remplit pas toutes les espérances, certaines mesures étant différées (la gratuité des médicaments pour les prestataires de l'aide sociale), d'autres détournées (l'indexation annuelle des prestations n'est que partielle pour les prestataires aptes au travail). Ces critiques ont été reprises en juin 2004, lors du dépôt par le gouvernement du projet de loi 57 qui proposait une réforme de l'aide sociale d'envergure. Plusieurs mesures de ce projet de loi ont été fortement décriées comme allant à l'encontre de la Loi 112, et comme remettant en question les gains obtenus pour les personnes démunies (réintroduction de la logique punitive, non-respect du principe de la prestation minimale, instauration de pratiques discriminantes) ${ }^{43}$. L'opposition au projet donnera lieu à des modifications, mais la Loi sur l'aide aux personnes et aux familles adoptée en juin 2005 confirmera la tangente précédente. L'accent est mis sur la valorisation du travail et les efforts d'insertion des personnes, et plusieurs des principes inscrits dans la Loi 112 ne sont toujours pas respectés. Entrée en vigueur le $1^{\text {er }}$ janvier 2007, la Loi sur

42. Marie-Renée Roy, Guy Fréchet, Frédéric Savard, «Travail et pauvreté au Canada», op. cit.

43. FCPASQ, Lutte à la pauvreté. Bilan des 100 premiers jours du gouvernement Charest, Conférence de Jean-Yves Desgagnés, 2003, p. 13-26; Collectif pour un Québec sans pauvreté, «Déclaration citoyenne: pour une sécurité du revenu couvrant les besoins dans la dignité et les droits», Communiqué de presse, 8 décembre 2004. 
l'aide aux personnes et aux familles remplace les programmes d'assistance-emploi en vigueur. Trois programmes sont instaurés: le Programme d'aide sociale; le Programme de solidarité sociale; et le Programme solidarité jeunesse ${ }^{44}$.

En juillet 2008, le gouvernement du Québec a annoncé une nouvelle orientation politique, le Pacte pour l'emploi ${ }^{45}$, qui vise à s'attaquer au volant des prestataires «irréductibles», autrement dit ceux qui demeurent prestataires en dépit des bonnes performances de l'économie. Publié conjointement par le ministère de l'Emploi et de la Solidarité sociale et le ministère de l'Éducation, des Loisirs et des Sports, le projet doit faciliter l'adéquation entre les besoins de maind'œuvre des entreprises et les bassins de main-d'œuvre disponibles. Très clairement, le travail est considéré dans ce pacte comme la base de la prospérité, sur une base individuelle autant que collective. Une première série de mesures vise «la mise en mouvement» rapide des nouveaux prestataires de l'aide sociale et un effort soutenu et ciblé vers les groupes davantage touchés par la pauvreté (comme les familles avec jeunes enfants ou les travailleurs âgés) ou prestataires de plus longue date; une autre vise l'intégration et le maintien en emploi des personnes handicapées; une autre, les entreprises (pour les inciter à l'embauche de personnes en situation de pauvreté). Au total, la logique d'intervention conduit essentiellement à «un accompagnement» le plus efficace possible pour répondre aux besoins du marché du travail, dans la continuité des décisions précédentes. On voit même, avec ce nouveau pacte, s'esquisser une approche où la formation professionnelle et la qualification sont reléguées

44. Québec, Répertoire des programmes: Services aux citoyens. Portail Québec, Services Québec, www.formulaire.gouv.gc.ca, 2008.

45. Québec, Le pacte pour l'emploi. Le Québec de toutes ses forces, www. pacte-emploi.gouv.qc.ca/presentation/index.asp. 
au second plan pour permettre une «mise au travail» immédiate et favoriser le bon fonctionnement du marché.

Ce n'est pas une tendance très nouvelle, mais plutôt la confirmation d'une évolution. En effet, depuis l'an 2000, les fonds du Québec consacrés à l'aide à l'emploi ont diminué de $16,6 \%$; on peut supposer que les mesures les plus onéreuses (subventions à l'embauche et formation, qui sont aussi celles qui réussissent le mieux pour les personnes les plus éloignées du marché du travail) ont été les premières à subir les compressions budgétaires ${ }^{46}$.

Comme on le voit, entre 1995 et 2007, le Québec a mis en place une série de mesures qui ont, partiellement, amélioré le sort des personnes les plus démunies. Les effets positifs de l'action publique ont également été servis par la conjoncture économique plutôt favorable. Mais pour bien saisir pourquoi le Québec est à la fois une société très inégalitaire, où les personnes pauvres représentent à peu près de manière constante $10 \%$ de la population, et en même temps LA province où la lutte contre la pauvreté a été la plus poussée, il nous faut retourner aux dimensions politiques qui sous-tendent le traitement de la pauvreté. Au-delà des normes institutionnelles et des politiques, il y a des acteurs en relation.

\section{À la manière du Québec}

Malgré les importants changements que le Québec a connus ces dix dernières années, il reste une société qui articule la quête d'une économie dynamique, un système politique fondé sur la coopération, un État protecteur et un partenariat

46. Pierre-Joseph Ulysse, «La lutte contre la pauvreté et les exclusions. Les paradoxes des stratégies québécoises», Informations sociales, $\mathrm{n}^{\circ}$ 143, 2007, p. 54-63. 
entre les différentes forces de la société ${ }^{47}$. Qu'est-ce que cela signifie? Reprenons brièvement les principaux ingrédients qui caractérisent la manière québécoise de faire de la politique ${ }^{48}$. Premièrement, il existe, au Québec, une relation État-société qui laisse une place significative à la concertation avec des acteurs collectifs, que l'on nomme parfois les «partenaires sociaux», à savoir les syndicats et les organisations patronales. Historiquement, on peut soutenir l'argument selon lequel le développement de l'État québécois s'est fait en parallèle avec le développement d'«organisations intermédiaires», en particulier les syndicats ${ }^{49}$. Cette relation se caractérise par deux aspects centraux. Le premier aspect concerne l'idée selon laquelle le Québec est le niveau d'action et de réflexion approprié, indépendamment de la distribution des pouvoirs entre le fédéral et le provincial. Cette constante de l'action politique constitue autant un réflexe pour les syndicats ${ }^{50}$ qu'un cadre «donné» de travail pour le patronat. Le deuxième aspect concerne les pratiques de concertation sur les relations de travail qui, bien que très décentralisées, sont tout de même soumises à un «regard étatique», qui peut aller jusqu'à des législations spécifiques ${ }^{51}$. Cette concertation est différente d'une approche de lobbying (même si elle ne l'exclut

47. Pierre-Joseph Ulysse, Frédéric Lesemann, Citoyenneté et pauvreté: politiques, pratiques et stratégies d'insertion en emploi et de lutte contre la pauvreté, Presses de l'Université du Québec, 2004.

48. Pascale Dufour, «L'adoption de la Loi 112 au Québec: le produit d'une mobilisation ou une simple question de conjoncture politique?», Politique et Sociétés vol. 23, nos 2-3, 2005, p. 159-182.

49. Mona-Josée Gagnon, «Syndicalisme et classe ouvrière. Histoire et évolution d'un malentendu », Lien social et Politiques, vol. 49, printemps 2003, p. 15-33.

50. Peter Graefe, «Nationalism and Competitiveness: Can Québec Win if Quebecers Lose?», Alain G. Gagnon (dir.), Quebec: State and Society, Broadview Press, 2004, p. 427-446.

51. François Delorme, Régis Fortin, Louis Gosselin, «L'organisation du monde patronal au Québec: Un portrait diversifié», Relations industrielles, vol. $49, \mathrm{n}^{\circ} 1,1994$, p. 9-38. 
pas), parce qu'il ne s'agit pas seulement de défendre des intérêts privés auprès de l'État, mais de mettre en place des mécanismes de coordination d'intérêts divergents, mécanismes qui font une place centrale à l'État ${ }^{52}$. On peut penser, en particulier, à la pratique des sommets socioéconomiques qui, depuis les années 1970, ont réuni le gouvernement et les partenaires sociaux pour décider des grandes orientations en matière de politiques économique et sociale.

Dans la période plus récente se sont également développés des secteurs de politiques publiques où les partenaires sociaux ont été étroitement associés aux décisions politiques et même, dans certains cas (comme dans le dossier de la formation de la main-d'œuvre), se sont vu confier une partie de la gestion et de la mise en œuvre des programmes, approfondissant l'institutionnalisation des mécanismes de concertation ${ }^{53}$. Cela dit, outre ces quelques exceptions, on ne trouve pas d'institutions tripartites au Québec, comme en France, où les partenaires sociaux gèrent, par exemple, le régime d'assurance-chômage. D'ailleurs, la concertation patronalesyndicale à l'échelle nationale est peu fréquente ${ }^{54}$, malgré le très fort taux de syndicalisation au Québec (plus de 40\%) et le regroupement du patronat à travers le Conseil du patronat du Québec (les négociations ont lieu au sein des entreprises pour les conventions collectives, mais davantage sur le mode de la conflictualité). La mise en forme de la concertation sociétale reste ainsi dépendante du bon vouloir des gouvernements en place, et son étendue reste limitée, le Québec

52. Éric Montpetit, «Pour en finir avec le lobbying: Comment les institutions canadiennes influencent l'action des groupes d'intérêts?», Politique et Sociétés, vol. 21, nº 3, 2002, p. 91-112.

53. Gilles L. Bourque, Le modèle québécois de développement. De l'émergence au renouvellement, Presses de l'Université du Québec, 2000.

54. Paul R. Bélanger, Benoît Lévesque, Le modèle québécois: corporatisme ou démocratie sociale?, CRISES/UQAM, 2001. 
demeurant une province canadienne dont beaucoup d'enjeux (notamment économiques) sont discutés et décidés en amont du cadre provincial. Plutôt qu'un modèle de type néocorporatiste, il est juste de parler d'une pratique récurrente de la concertation, non institutionnalisée et donc discrétionnaire, offrant néanmoins des marges de manœuvre concrètes aux partenaires ${ }^{55}$.

Le deuxième ingrédient-clef concerne la relation particulière qui s'est développée entre l'État et les organismes communautaires. Notamment, la mise en place progressive des tables de concertation dites sectorielles a permis d'offrir un cadre d'action où la consultation des groupes n'était plus sporadique, mais constituait un véritable travail de concertation avec l'État. Dans les années 1990, ce travail de concertation n'est plus seulement vrai au palier sectoriel. Les groupes communautaires travaillent de plus en plus conjointement sur des dossiers trans-sectoriels ou multisectoriels, par exemple les questions du logement ou de la pauvreté. Le rôle central des acteurs communautaires dans le processus de décision publique constitue aujourd'hui une caractéristique distincte du Québec ${ }^{56}$, dont le symbole pourrait être l'adoption, en 2001, de la Politique de reconnaissance et de

55. Gilles Paquet, «Québec Inc.: Mythes et réalités», Filip Palda (dir.), L'État interventionniste: le gouvernement provincial et l'économie du Québec, Fraser Institute, 1994; Michael R. Smith, «L'impact de Québec Inc., répartition des revenus et efficacité économique», Sociologie et sociétés, vol. 36, $\mathrm{n}^{\circ} 2$, 1994, p. 91-110. Précisons que cette pratique de la concertation n'exclut en rien les relations conflictuelles qui peuvent exister entre les centrales syndicales et les gouvernements successifs du Québec. Voir Roch Denis, Serge Denis, Les syndicats face au pouvoir. Syndicalisme et politique au Québec de 1960 à 1992, Vermillon, 1992.

56. Deena White, «Contradictory Participation: Reflections on Community Action in Quebec», Michael Elague, Brian Wharf (dir.), Community Organizing Canadian Experiences, Oxford University Press, 1997; Jane Jenson, «Les réformes des services de garde pour jeunes enfants en France et au Québec: une analyse historico-institutionnaliste», Politiques et Sociétés,

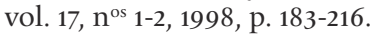


soutien de l'action communautaire, qui définit leur place en tant que «partenaires» de l'État et leur assure un cadre de financement plus ou moins pérenne. Les travaux de Laforest ont par ailleurs bien montré qu'il ne s'agissait pas seulement de réseaux de politiques publiques, mais bien de «réseaux civiques» qui mettaient en relation de nombreux acteurs de la société civile dans un maillage très complexe, dépassant largement la compartimentation par champs de politiques publiques particuliers ${ }^{57}$.

Pour ces acteurs, la relation à l'État du Québec est centrale (c'est lui qui est la cible des demandes et non l'État fédéral), mais l'existence de ces réseaux permet aussi le partage d'informations entre eux (qui ont des niveaux de ressources différents), rend possibles des mobilisations communes sur des sujets transversaux (comme la pauvreté ou la justice sociale) et fournit les ingrédients nécessaires pour que ces acteurs puissent développer, à des moments spécifiques, un rapport de force avec l'État qui compte réellement. En bref, le «partenariat» n'existe pas entre trois partenaires, comme dans la plupart des sociétés dites «néocorporatistes» ou de concertation, mais entre quatre, incluant les groupes communautaires. Dans les années 1990, ces partenaires ne seront plus seulement sollicités à titre de collaborateurs du développement social, mais également pour leur expertise ${ }^{58}$. La possibilité de ce nouveau statut au sein de l'État a infléchi, sans aucun doute, les stratégies d'action collective au cours des années 1990 et explique, en partie, l'initiative du Collectif de proposer une loi ainsi que son succès.

57. Rachel Laforest, «La consultation publique et l'action collective», Politique et Sociétés, vol. 19, nº 1, 2000, p. 3-30.

58. Dominique Masson, «Gouvernance partagée, associations et démocratie: les femmes dans le développement régional», Politique et Sociétés,

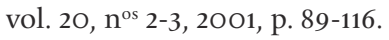


La politisation de l'enjeu de la lutte contre la pauvreté et les décisions publiques qui ont suivi n'ont pourtant pas enrayé le phénomène. La société québécoise, par ces compromis sociaux et politiques, s'accommode tout de même de laissés-pour-compte de plus en plus pauvres.

\section{Les laissés-pour-compte}

Le Québec, comme le Canada et la Grande-Bretagne, a hérité d'un passé de régime libéral qui considère que l'État social doit s'occuper, minimalement, des plus démunis et non intervenir comme garant de la solidarité sociale. Cette histoire d'État social charitable s'accompagne d'une représentation sociale tenace de la pauvreté divisée entre bons et mauvais pauvres. Elle traverse toute l'histoire du traitement politique de la pauvreté au Québec ${ }^{59}$. Ainsi, cette représentation de la pauvreté, qui culpabilise et fait peser la responsabilité de la pauvreté sur les seules épaules des individus, a des répercussions sur la perception qu'ont les Canadiens et les Québécois de l'aide publique qu'on peut leur apporter. Un sondage national effectué en 1994 révèle que 59\% des répondants étaient d'accord pour que l'on réduise les dépenses d'assistance sociale, alors que $36 \%$ seulement s'y opposaient. Les compressions effectuées dans le budget de l'assistance sociale recueillaient le plus d'appuis, suivies de celles qui touchaient l'assurance-chômage ${ }^{60}$.

Néanmoins, les représentations sociales de la pauvreté ont changé: en 2009, les représentations culpabilisantes et même suspicieuses par rapport aux prestataires de l'aide

59. Sylvie Morel, Modèle du workfare ou modèle de l'insertion? La transformation de l'assistance sociale au Canada et au Québec, Condition féminine Canada, dsp-psd.tpsgc.gc.ca/Collection/SW21-95-2002F.pdf.

6o. Nicole F. Bernier, Le désengagement de l'État-providence, Presses de l’Université de Montréal, 2003. 
sociale sont devenues minoritaires. Aujourd'hui, le gouvernement du Québec aborde la pauvreté comme une notion complexe ayant des effets négatifs sur la croissance économique, des coûts dus à la non-utilisation des ressources humaines, des répercussions négatives sur la cohésion sociale et, enfin, des retombées sur la participation à la vie démocratique et sur l'égalité des chances ${ }^{61}$. Plusieurs facteurs expliquent cette transformation. On peut raisonnablement penser que la forte mobilisation sociale en faveur de la lutte contre la pauvreté et l'exclusion porte ses fruits. Aussi, le gouvernement du Québec reconnaît que la pauvreté a des impacts directs sur la santé des populations et donc sur le budget de l'État. Personne, finalement, même en termes comptables, n'a intérêt à ce que les Québécois soient pauvres. En revanche, les inégalités sociales persistantes, voire croissantes, ne sont pas devenues un enjeu de débat public et peu de mesures ont été prises pour en corriger les effets. Le débat public par rapport aux inégalités s'est davantage structuré autour des inégalités de santé des populations (voir les très nombreux rapports gouvernementaux sur cette question, la multiplication des centres de recherche), mais les inégalités socioéconomiques sont rarement questionnées en tant que telles. Pourtant, selon le tableau ci-dessous, les personnes pauvres sont de plus en plus pauvres.

Comme on le voit, la société québécoise était plus égalitaire au milieu des années 1990 qu'elle ne l'est aujourd'hui, où $20 \%$ des plus riches détiennent quasiment $40 \%$ des revenus disponibles, alors que $20 \%$ des plus démunies n'en détiennent que 5,4\%.

61. Pierre-Joseph Ulysse, Frédéric Lesemann, Citoyenneté et pauvreté: politiques, pratiques et stratégies d'insertion en emploi et de lutte contre la pauvreté, Presses de l’Université du Québec, 2004. 
Certes, les mesures mises en œuvre témoignent d'une prise de conscience que la nature de l'emploi et la qualité de celui-ci sont des facteurs déterminants pour «sortir» de la pauvreté en même temps que de l'aide sociale. Un effort constant a d'ailleurs été noté pour redresser le niveau de revenu des emplois au salaire minimum. Mais ces mesures ne règlent pas tout. Le marché du travail lui-même produit de l'insécurité qui dépasse de loin le contrôle que le travailleur peut avoir sur son parcours de vie. Tout miser sur l'emploi (y compris l'emploi à faible qualification, comme dans le dernier pacte pour l'emploi) pour lutter contre la pauvreté et l'exclusion sociale, c'est oublier le fait que les individus circulent au sein de structures sociales inégales auxquelles nous pourrions aussi nous attaquer. Un recul sur le front de l'emploi et combien de personnes basculeront à nouveau?

Statistiquement, l'action volontariste de l'État en direction des plus démunis, couplée à la forte progression de l'emploi dans la période 1996-2006, a permis de résorber les effectifs de l'aide sociale. Globalement, selon le Rapport annuel de gestion 2005-2006 de la Sécurité du revenu, le taux d'assistance sociale est passé de 11,8\% en 1995-1996 à 7,7\% en 2005-2006. Le nombre d'adultes recevant une aide de dernier

TABLEAU 5.3

Part du revenu disponible détenu par les différents quintiles, selon le type de famille économique, 1986-2002

\begin{tabular}{|l|c|c|c|c|c|}
\hline Familles & Quintile 1 & Quintile 2 & Quintile 3 & Quintile 4 & Quintile 5 \\
\hline 1986 & 7,7 & 13,5 & 18,3 & 24,1 & 36,4 \\
\hline 1993 & 8,2 & 13,8 & 18,4 & 24,1 & 35,6 \\
\hline 1994 & 8,1 & 13,8 & 18,6 & 23,9 & 35,7 \\
\hline 2002 & 5,4 & 12,2 & 18,1 & 24,7 & 39,5 \\
\hline
\end{tabular}

Source: Statistique Canada, Enquête sur les finances des consommateurs (EFC) et enquête sur la dynamique du travail et du revenu (EDTR), compilé par l'Institut de la statistique du Québec. 
recours est même passé deux mois consécutifs sous le seuil de 500 ooo personnes, un creux non atteint depuis décembre 1979. Au chapitre du soulagement de la pauvreté, dans l'ensemble le bilan apparaît positif pour les familles avec enfants $^{62}$, y compris certaines familles de parents seuls (taux d'emploi en hausse et taux de faibles revenus en baisse). Selon une étude citée par Roy, Fréchet et Savard ${ }^{63}$, les mesures d'aide à la famille sont parmi les principaux facteurs expliquant la baisse des familles monoparentales à l'aide sociale. En revanche, la situation des personnes seules apparaît beaucoup moins enviable; elles ont moins que les autres profité des nouvelles mesures mises en place et continuent de constituer un groupe particulièrement vulnérable à la pauvreté ${ }^{4}$. Elles comptent pour presque la moitié des personnes en situation de pauvreté et ont également tendance à être les plus pauvres, parmi les personnes pauvres. Les personnes seules sont soit des hommes d'âge mûr (entre 40 et 55 ans), soit des personnes âgées. Les familles monoparentales - dont la majorité sont dirigées par des femmes -, les ménages qui ont un chef de moins de 25 ans, sont quelquesunes des autres catégories de population ayant plus de probabilité de connaître des épisodes de pauvreté ${ }^{65}$.

Enfin, une étude de Picot et Feng Hou ${ }^{66}$ montre qu'au cours des deux dernières décennies, le taux de faible revenu

62. Alain Noël, Priorité au soutien du revenu: la mise en œuvre de la Loi québécoise visant à lutter contre la pauvreté et l'exclusion sociale, Canadian Policy Research Networks, 2004.

63. Marie-Renée Roy, Guy Fréchet, Frédéric Savard, «Travail et pauvreté au Canada», op. cit.

64. Gouvernement du Québec, Le faible revenu au Québec: un état de la situation, Centre d'étude de la pauvreté et de l'exclusion, 2008.

65. Michel Laffut, Marie-Renée Roy, Pauvreté et exclusion sociale. Partage d'expériences entre Wallonie et Québec, De Boeck, 2007, Introduction.

66. Garnett Picot, Feng Hou, The rise in low income rates among immigrants in Canada, Statistique Canada, www.statcan.gc.ca/bsolc/olc-cel/olc-cel?cat no=11FoO19M2OO3198=eng. 
a baissé chez les Canadiens de naissance et augmenté chez les immigrants, renforçant l'idée d'une répartition inégale de la pauvreté et des risques qui y sont associés. Les femmes immigrantes apparaissent, au sein des nouveaux arrivants, les personnes les plus vulnérables ${ }^{67}$.

\section{Conclusion}

Le cas du Québec, comme le cas britannique, nous rappelle qu'il ne faut pas confondre «sortir de l'aide sociale» et «sortir de la pauvreté». Le défi pour beaucoup de travailleurs n'est pas tant de trouver du travail que de décrocher un emploi de qualité, offrant une certaine sécurité (sur le plan financier, de la durée du travail, mais aussi et par ricochet, sur le plan psychologique). Au Québec, avec la hausse du salaire minimum, on a reconnu la nécessité de combattre la pauvreté au travail. Cependant, les autres interventions publiques consistent davantage à rendre disponible la main-d'œuvre pour favoriser le fonctionnement le plus efficace possible du marché du travail. En effet, il ne s'agit plus seulement d'offrir des formations à des travailleurs qualifiés, ou des plans d'accompagnement à des personnes éloignées du marché du travail; il s'agit de fournir de la main-d'œuvre aux employeurs qui en ont besoin. Cette logique d'intervention est particulièrement claire dans le dernier pacte pour l'emploi (2008).

Dans ce contexte où «la fourniture de main-d'œuvre» devient l'objectif premier des politiques sociales, ce qu'on risque de voir se développer de plus en plus, ce sont des mesures différenciées envers les plus démunis. N’étant plus

67. Pierre Myrlande, «Les facteurs d'exclusion faisant obstacle à l'intégration socioéconomique de certains groupes de femmes immigrées au Québec. Un état des lieux», Nouvelles pratiques sociales, vol. 17, $\mathrm{n}^{\circ} 2,2005$, p. 75-94. 
seulement des «sans-emploi», mais un bassin de maind'œuvre qu'il faut mettre en mouvement, la nature de la mise en «mouvement» des prestataires va varier suivant le profil du prestataire. On peut alors facilement imaginer des parcours de vie très distincts suivant les personnes. Aux «irréductibles» chômeurs, le travail avec faible qualification; aux nouveaux chômeurs, un retour accompagné au travail par le biais d'une formation de court terme; et aux chômeurs qualifiés, une formation encore plus qualifiante. Finalement, le Québec nous montre que l'action collective et l'action publique, quand elles vont dans le même sens, peuvent modifier les fondements hérités de la pauvreté.

Depuis la Deuxième Guerre mondiale, on peut repérer, de manière grossière, trois séquences dans l'évolution des prérogatives sociales de la plupart des États occidentaux. À une première phase favorable au développement de l'État social aurait succédé au début des années 1980 une séquence marquée, au contraire, sous l'égide d'un tournant néolibéral, par un retrait, voire une volonté de démantèlement, de l'État social. Enfin, depuis la fin des années 1990, une troisième séquence est caractérisée, dans le sillage des réformes initiées par l'administration Clinton et la $3^{\mathrm{e}}$ voie de Tony Blair, par la promotion d'un État «d'investissement social». L'«activation» des dépenses sociales y tient une place prépondérante, mettant la formation, la qualification et la mise à l'œuvre de la main-d'œuvre au cœur des dispositifs de lutte contre la pauvreté. Entre la carotte et le bâton, entre le workfare et le welfare, entre la consolidation de dispositifs collectifs assistanciels (notamment pour lutter contre la pauvreté des enfants) et l'accentuation de la responsabilité individuelle, la plupart des politiques sociales des États s'alignent 
aujourd'hui sur la promotion d'un «juste milieu». Celui-ci conjuguerait les vertus des effets d'apprentissage croisés et la découverte d'un point d'équilibre entre les différentes contraintes et ambitions des politiques sociales. D'ailleurs, de l'Union européenne à l'OCDE, en passant par les professions de foi des différents pays en matière sociale, les mêmes recettes sont mises en avant, sur le mode de «bonnes pratiques» à adopter et de cibles chiffrées à atteindre. Assisterait-on dans le domaine des politiques sociales à la «fin de l'Histoire»?

Le tableau que nous avons dépeint de l'opposition idéaletypique entre les configurations danoise et britannique constitue un démenti net, même si elles sont parfois associées au titre de l'activation de la main-d'œuvre. Mais ce sont également des cas plus hybrides, comme les configurations françaises et québécoises, qui révèlent des différences importantes dans les modalités d'accommodement de la pauvreté.

Alors, comment instruire le débat récurrent sur la convergence ou la divergence des déclinaisons nationales d'État social? Dans les deux derniers chapitres, nous abordons de front cette question. En premier lieu, nous montrons comment les termes du débat sont souvent mal posés, notamment lorsqu'ils se focalisent sur la fétichisation d'indicateurs statistiques hégémoniques. En effet, les outils de mesure retenus pour forger le diagnostic sur la situation socioéconomique d'un pays (taux de chômage, taux de croissance du PIB) provoquent d'importants effets de myopie, voire de cécité. Plus encore, loin d'être de simples appendices techniques, leur emploi recoupe des enjeux normatifs. Ils sont actuellement des pierres angulaires du jeu politique car ils sont utilisés comme preuve d'une convergence des politiques sociales, devenue une injonction normative et promue au travers d'accords institutionnels interétatiques mais aussi d'étalonnage des «bonnes pratiques» (benchmarking), comme 
dans le cas européen. Aussi, l'affichage des «bons indicateurs» permet la qualification ou la disqualification d'un État. On démontre donc comment l'instruction du débat de la convergence/divergence sur la seule foi de ces indicateurs introduit une réponse fortement biaisée.

Puis, dans le dernier chapitre, nous contribuons à une reformulation du débat en questionnant l'objet de la convergence/divergence. Plutôt que d'évaluer la convergence/ divergence des États, des indicateurs ou des outils utilisés, nous préférons nous intéresser à la convergence/divergence des formes de pauvreté et des modalités d'accommodements qui les accompagnent. À ce titre, si la pauvreté et les inégalités existent partout, elles prennent des formes différentes selon les configurations nationales et on n'assiste pas à une convergence de celles-ci. Surtout, la morphologie des formes de pauvreté ne prend sens qu'au regard de son inscription au sein de la pyramide que nous avons circonscrite, constituée des dynamiques en cours sur le marché du travail, de l'architecture des compromis sociaux institutionnalisés, du traitement politique de la pauvreté et des dimensions symboliques attachées à cette question sociale. C'est à travers ce prisme qu'une lecture comparative des fondements nationaux de la pauvreté prend tout son sens. 



\section{(Dé)chiffrer la pauvreté : des instruments d'objectivation en débats}

La mesure statistique de la pauvreté est aujourd'hui au cœur d'un débat complexe. Tout d'abord, c'est le lien entre politique et statistique qui fait problème, avec le danger d'une instrumentalisation de la statistique par le pouvoir. Ensuite, c'est la question de la pertinence des indicateurs utilisés qui est questionnée. À des fins de clarté de la démonstration, nous allons préserver la distinction analytique entre ces deux dimensions du débat et les explorer tour à tour. Mais intrinsèquement, ces deux questions de «l'instrumentation» (la genèse d'indicateurs statistiques) et de «l'instrumentalisation» (leur utilisation stratégique) sont imbriquées. Derrière les controverses techniques, la genèse d'un indicateur statistique est toujours solidaire d'une démarche politique.

\section{La faute aux indicateurs? Enjeux de la quantification}

Dans La politique des grands nombres ${ }^{1}$, Alain Desrosières démontre ainsi que derrière la dualité des débats sur les

1. A. Desrosières, La politique des grands nombres, histoire de la raison statistique, La Découverte, 1993. 
indicateurs statistiques (d'un côté sur leur fiabilité, de l'autre sur la définition de ce qu'ils mesurent) s'articulent de manière indissociable leurs fonctions cognitives et pratiques. La proposition théorique de l'auteur est de «penser en même temps que les objets existent bien et que cela n'est qu'une convention». Substituant ainsi la question de l'objectivation à celle de l'objectivité, il invite à considérer l'ensemble des opérations qui conduisent à représenter un phénomène via la «raison statistique»: cet «espace cognitif d'équivalence et de comparabilité construit à des fins pratiques ${ }^{2} »$. Cette imbrication entre fonctions descriptive et performative, comme le suggère la locution "prendre des mesures», se donne à voir dans la genèse d'instruments statistiques liés à celle de formes particulières d'État social. L'étude comparée de l'Allemagne, de la France, de la Grande-Bretagne et des ÉtatsUnis, menée par Desrosières, témoigne de la relation entre configuration politico-administrative et type d'enquête valorisée. Loin d'être un outil neutre, la statistique sociale permet la représentation d'un phénomène, sa construction sociale - au sens fort - et sa mise en visibilité. Or, comme le montrent Rueschmeyer et Skocpol dans leur étude de la genèse des politiques sociales ${ }^{3}$, la création de savoirs sociaux par les institutions étatiques - au premier plan desquelles la statistique - permet la constitution de domaines d'intervention légitimes pour les administrations modernes.

2. Ibid., p. 399. Pour une histoire de la comptabilité nationale en France, avec un accent mis sur l'importance des conventions politiques qui la fondent, voir F. Fourquet, Les comptes de la puissance, Encres, 1980. Pour une histoire mettant davantage l'accent sur les controverses scientifiques, voir A. Vanoli, Une histoire de la comptabilité nationale, La Découverte, 2002.

3. Dietrich Rueschemeyer, Theda Skocpol, States, Social Knowledge, and the Origins of Modern Social Policies, Princeton University Press, Russell Sage Foundation, 1996. 
Dans les premières pages de cet ouvrage, nous avons souligné les débats techniques autour de la définition des seuils de pauvreté, et les conséquences politiques du choix de tel ou tel seuil (par exemple quand la pauvreté semble «doubler» par un simple changement de seuil dans les modalités de calcul). Mais plus fondamentalement, c'est à la fois le fondement artefactuel de tout agrégat statistique et, corrélativement, le pouvoir d'objectivation de l'instrument - au point que la chose semble exister "par elle-même» - qu'il faut avoir à l'esprit. L'institutionnalisation des outils statistiques, sur le mode de la naturalisation, est d'ailleurs liée à l'anamnèse, c'est-à-dire l'oubli des conditions de sa genèse. L'aboutissement d'une construction statistique et administrative est de faire exister des catégories comme «le chômage», «la pauvreté» ou «la croissance» comme des «choses sociales» dont l'existence et la signification semblent aller de soi pour les citoyens et les décideurs publics. Mais cette naturalisation tient à des conditions fragiles et la consécration d'un instrument comme simple «reflet de la réalité» peut vaciller, comme le montrent les controverses actuelles sur le PIB dont la prétention à être l'étalon universel de mesure de la richesse des nations est vivement discutée. Privilégier tel ou tel instrument conduit à dessiner un portrait différent de la réalité socioéconomique, en prenant en compte d'autres dimensions.

Certaines séquences historiques et certaines configurations politiques peuvent rendre plus aiguë cette recherche d'indicateurs permettant de connaître «véritablement» la société. Ce fut le cas par exemple du gouvernement fédéral américain face à la crise des années 1930, démuni d'outils pouvant mesurer le nombre de chômeurs ou leur répartition sur le territoire. Il dut investir dans des dispositifs statistiques novateurs pour à la fois savoir «en quoi consiste 
l'Amérique» et pouvoir mettre en place les politiques publiques du New Deal ${ }^{4}$. Ce fut le cas également en France ces dernières années, alors que la répétition des sondages portant sur le sentiment des ménages français d'une vive dégradation de leur pouvoir d'achat face à une augmentation des prix, régulièrement contredits par les données sur la stabilité de l'indice des prix, amena le gouvernement à incriminer les mesures de l'Insee comme ne reflétant plus «la réalité» ${ }^{5}$.

Cette mise en exergue de l'artificialité de la construction de l'agrégat statistique prend une importance spécifique dans la configuration actuelle. Aujourd'hui, l'apparition d'instruments comme «l'empreinte écologique» ou encore «le bilan carbone» suscite le trouble dans l'ordre socioéconomique, en objectivant un phénomène auparavant absent des comptabilités standardisées des États-nations. La prise en compte d'un ensemble d'externalités négatives du développement économique amène donc à réindexer les classements et diagnostics sur la soutenabilité des modèles de croissance. Cette remise en question de la justesse du diagnostic produit par les instruments de mesure jusqu'ici hégémoniques, tel le $\mathrm{PIB}$, invite à reconsidérer en quoi consiste la richesse (et la pauvreté) des nations.

On le perçoit sans peine, la représentation de «la réalité» via l'objectivation statistique (dire «ce qui compte» et «comment on le compte») est donc un travail indissociablement politique et scientifique. Dans le domaine des politiques sociales, «prendre des mesures adéquates» concernant la pauvreté et les inégalités sociales recouvre des enjeux cruciaux. D’une part, parce que la séquence historique dans

4. Emmanuel Didier, En quoi consiste l'Amérique? Les statistiques, le New Deal et la démocratie, La Découverte, 2009.

5. Lorraine Data, Le grand truquage. Comment le gouvernement manipule les statistiques, La Découverte, 2009. 
laquelle nous évoluons est propice à la remise en question des instruments d'objectivation du social, dont témoigne le retour en grâce d'instruments alternatifs de mesure de la pauvreté et de la richesse. D'autre part, parce que la quantification du social devient un enjeu politique à l'heure où le benchmarking, c'est-à-dire les pratiques d'étalonnage de performances, devient un art de gouvernement. C'est sur ces deux dimensions que nous allons revenir tour à tour.

\section{Mesure de la pauvreté et pauvreté de la mesure}

Depuis une vingtaine d'années, un certain nombre d'indicateurs socioéconomiques alternatifs ont été conçus pour contrer l'hégémonie du PIB. Dans un ouvrage de synthèse, Jean Gadrey et Florence Jany-Catrice reviennent sur ces instruments, qu'ils considèrent, à rebours d'une vision purement technicienne, comme «des formes de connaissances qui dépendent de conventions politiques et de systèmes de valeurs ${ }^{6} »$. Ils les envisagent comme «des conventions (des cadres cognitifs et éthiques) et des outils de régulation (des cadres institutionnalisés de l'action publique et privée) $)^{7}$. En prenant à bras-le-corps à la fois la construction technique des indicateurs et en élucidant les soubassements normatifs structurant tel ou tel instrument, ils ouvrent ces véritables «boîtes noires» de l'environnement politique et scientifique. Dépassant une opposition factice entre «objectivité scientifique» et «normativité politique», ils proposent de rendre transparentes la «discutabilité» (au sens propre) et les implications des modalités de composition des principaux indicateurs statistiques synthétiques, qu'ils soient hégémoniques

6. Jean Gadrey, Florence Jany-Catrice, Les nouveaux indicateurs de richesse, La Découverte, 2005, p. 6.

7. Ibid., p. 7. 
(PIB, taux de croissance) ou alternatifs (IDH du PNUD, PIB vert, IBED, empreinte écologique, etc.).

Par rapport aux premiers nommés, les indicateurs alternatifs ont en commun de contester la capacité du PIB à déchiffrer l'état de la pauvreté et de la richesse d'un pays. La critique est double: d'une part, le PIB (et le taux de croissance économique, autre étalon fétiche) ne mesure pas le «progrès social», d'autre part, il est aveugle à un certain nombre d'éléments, et notamment à la question environnementale. Le paradigme spécifique porté par le PIB est le suivant: la croissance économique réglera la question sociale. Or, cette vision conduit à de nombreuses apories. La première est qu'une amélioration du PIB ne dit rien sur «ce qui s'est amélioré» précisément, ni sa répartition (partage de la valeur ajoutée). Second élément, le PIB ne prend pas en compte «les dégâts» de la croissance enregistrée. Gadrey et Jani-Catrice donnent ainsi l'exemple d'une société qui aurait beaucoup d'accidents de la route, et qui serait donc dotée d'un meilleur PIB grâce à l'activité ainsi générée (soins, réparations de voiture, etc.), toute chose égale par ailleurs, qu'une société où les gens conduisent prudemment. Pourtant, il serait absurde de considérer que la première génère un «surplus de bien-être» par rapport à la seconde. La même idée peut être développée à propos des dépenses «défensives» déployées pour réparer les dégâts environnementaux. Se pose alors la question des externalités négatives générées par le modèle économique, mais qui ne sont pas prises en compte dans le calcul de la richesse et de la pauvreté d'une société. Troisième aporie d'une mesure uniquement centrée sur le PIB: l'absence de prise en compte du travail domestique, du bénévolat, bref d'un ensemble de contributions, notamment dans le secteur des soins et de l'entraide (Care), pourtant centrales sur les questions de pauvreté. 
De même, la fétichisation du «chiffre du chômage» subit de multiples remises en question. Comme nous l'avons montré, les formes de l'activité salariée ont profondément changé ces dernières années. Signe de ce décalage entre indicateurs et réalités observées, la persistance du vocable d'«emploi atypique» alors que ce type de contrat constitue aujourd'hui la norme, parmi les contrats le plus souvent proposés. Alors, de quel chômage parle-t-on? Et quid des travailleurs pauvres? Quel indicateur statistique mesure aujourd'hui la précarité dans les tableaux d'indicateurs nationaux? En France, la dissolution d'un organisme comme le Centre d'études sur les revenus et les coûts (CERC), ou encore la difficulté à susciter de la part de l'Insee des statistiques sur le poids des bas salaires ou de la pauvreté, illustrent cette «timidité» des gouvernements à l'endroit des instruments d'objectivation précis de ces phénomènes sociaux.

Plusieurs instruments alternatifs ont d'ailleurs été développés par des réseaux à la fois universitaires et militants, proches des courants altermondialistes, à l'image du baromètre des inégalités et de la pauvreté, en France, dit le BIP 40 (contraction et détournement, sous forme de clin d'œil, du «PIB» et du «CAC 4O») ${ }^{8}$. Créé au début des années 2000, l'objectif de cet indicateur est d'établir de manière globale une mesure synthétisant l'évolution des inégalités et de la pauvreté en France. À ce titre, le BIP 40 agrège différents indicateurs existants, dans les domaines de l'emploi (24 indicateurs concernant notamment le taux de chômage, mais aussi la part des chômeurs de longue durée, la précarité, les conditions de travail, les relations professionnelles, etc.), des

8. Pour un retour sur l'histoire du BIP 40, voir l'interview d'un de ses promoteurs, dans «Les indicateurs économiques alternatifs. L'exemple du BIP 40", Savoir/Agir, Revue trimestrielle de l'association Savoir/agir, vol. 8, juin 2009, p. 43-56. 
revenus (15 indicateurs sur les salaires, la pauvreté, les inégalités et la fiscalité et la consommation), de la santé (5 indicateurs dont l'espérance de vie, mais aussi la différence entre celle des cadres et celle des ouvriers), de l'éducation (5 indicateurs sur l'inégalité des performances scolaires), du logement (5 indicateurs), et enfin de la justice (4 indicateurs, dont la part de la population incarcérée par rapport à la population totale) ${ }^{9}$. Si la consultation d'un tableau de bord exhaustif, avec une multiplicité d'indicateurs, est évidemment plus précise et plus riche, les initiateurs du projet ont cependant souhaité forger un indicateur global et synthétique, conscients du poids politique du chiffre unique, par rapport à une myriade d'indices. Ils souscrivent ainsi pour partie à la vertu du fétichisme du chiffre, tirant davantage sa force de son pouvoir d'évocation («il a doublé», «il a perdu un point»...) que de sa précision scientifique ${ }^{10}$.

Au Canada, les travaux d'Osberg et Sharpe ${ }^{11}$ ont conduit à la formalisation d'un «indice de bien-être économique» qui présente deux particularités intéressantes pour notre propos. Tout d'abord, il prend en considération un certain nombre d'éléments d'habitude «hors cadre» (travail domestique et bénévolat, patrimoine de ressources naturelles par tête), en leur octroyant un équivalent monétaire. De plus, il met l'accent, parmi les différentes dimensions du bien-être

9. Jean Gadrey, Florence Jany-Catrice, Les nouveaux indicateurs de richesse, La Découverte, 2005, p. 38-41. Voir aussi BIP4O, baromètre des inégalités et de la pauvreté, www.bip40.org/.

10. On n'est pas très loin ici du sondage, qui tire sa force politique de son pouvoir de dire de manière synthétique et «scientifique», en quoi consiste «l'opinion publique». Voir Loïc Blondiaux, «Le chiffre et la croyance. L'importation des sondages d'opinion en France ou les infortunes d'une opinion sans publics», Politix - Revue des sciences sociales du politique, vol. 25, 1994, p. 117-152.

11. Lars Osberg, Andrew Sharpe, «New Estimates of the Index of Economic Well-being for Selected OECD Countries, 1980-2007», CSLS Report, 2009. Voir aussi CSLS, Centre for the Study of Living Standards, www.csls.ca, 2009. 
FIGURE 6.1

\section{L'indice de bien-être économique d'Osberg et Sharpe}

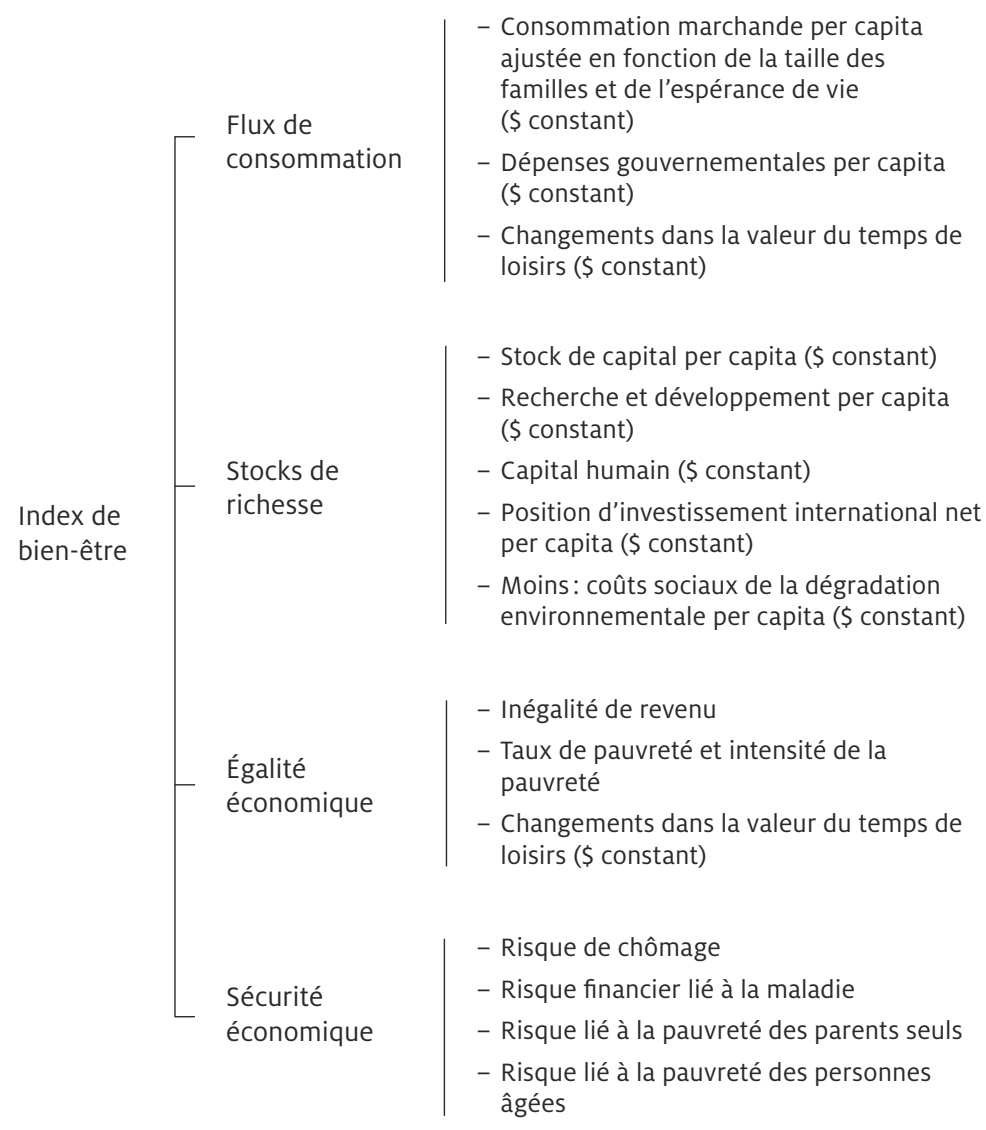

Source: Lars Osberg, Andrew Sharpe, « New Estimates of the Index of Economic Well-being for Selected OECD Countries, 1980-2007», CSLS Report, décembre 2009, p. 6. 
GRAPHIQUE 6.1

Taux de croissance annuels de l'IBEE (Indice de bien-être économique) et du PIB, pays de l'OCDE, 1980-2007

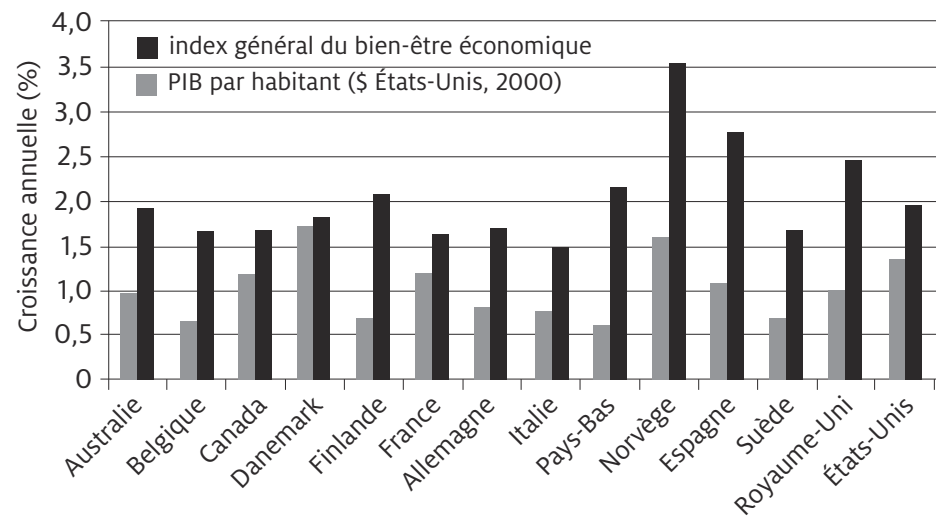

Source: Lars Osberg, Andrew Sharpe, «New Estimates of the Index of Economic Well-being for Selected OECD Countries, 1980-2007 », CSLS Report, décembre 2009, p. 13. Les données proviennent de l'OCDE.

économique, sur le degré de sécurité sociale (ou de protection sociale) ${ }^{12}$. L'insécurité économique est donc évaluée par la mesure des risques objectifs liés au chômage, à la maladie, au veuvage (et à la situation monoparentale) et à la vieillesse, comme on le voit sur la quatrième «branche» de ce schéma.

Mesurer le développement des pays à l'aune de leur BIP ou de cet indice alternatif permet de mettre à jour de manière synthétique certains contrastes, que nous avons rencontrés de manière plus transversale dans les chapitres qui précèdent.

12. Pour une synthèse de ce modèle d'évaluation, voir Jean Gadrey, Florence Jany-Catrice, Les nouveaux indicateurs de richesse, op. cit. Pour une présentation et une discussion détaillées, voir Lars Osberg, Andrew Sharpe, «Measuring Economic Security in Insecure Times: New Perspectives, New Events, and the Index of Economic Well-being», CSLS Report, 2009. 
On mesure à la fois l'écart entre l'accroissement du «bienêtre économique», tel que les auteurs l'entendent, et la croissance économique, au sens généralement entendu via la mesure du BIP. Mais on saisit également de manière directe à quel point les modèles socioéconomiques des pays divergent, par exemple entre d'un côté le Royaume-Uni et de l'autre le Danemark et la France; si le premier a un taux de croissance du BIP plus important que les seconds sur la période 1980-2007, la croissance de son IBEE est nettement inférieure.

La même mise en perspective à propos des provinces canadiennes permet de mettre en exergue la spécificité du modèle socioéconomique québécois.

Une comparaison de ces deux mesures, non plus synchronique, mais diachronique, permet, au-delà d'un simple diagnostic d'«amélioration générale», de mettre en lumière précisément ce qui s'est amélioré, mais aussi ce qui a pâti sur une séquence socioéconomique, telle que celle qu'a connue le Canada depuis le début des années 1980.

Ces quelques illustrations témoignent du pouvoir herméneutique de ce type d'instruments d'objectivation statistique. Ils témoignent à la fois d'avancées scientifiques et d'angles d'analyse politiques féconds pour mieux déchiffrer en quoi consistent la richesse et la pauvreté des nations. Ces indicateurs sont d'ailleurs de plus en plus utilisés par des organisations comme l'OCDE, la Banque mondiale ou l'ONU. En France, la parution, à l'invitation du président Sarkozy, du «rapport Stiglitz», témoigne du succès nouveau de ce type d'instruments.

En février 2008, le président français a demandé à Joseph Stiglitz (président de la commission), Amartya Sen (conseiller) et Jean-Paul Fitoussi (coordinateur), de mettre en place la Commission pour la mesure des performances économiques et du progrès social. Il s'agissait de cerner les limites 
GRAPHIQUE 6.2

Taux de croissance annuels de l'IBEE (Indice de bien-être économique) et du BIP, Canada et provinces, 1981-2008

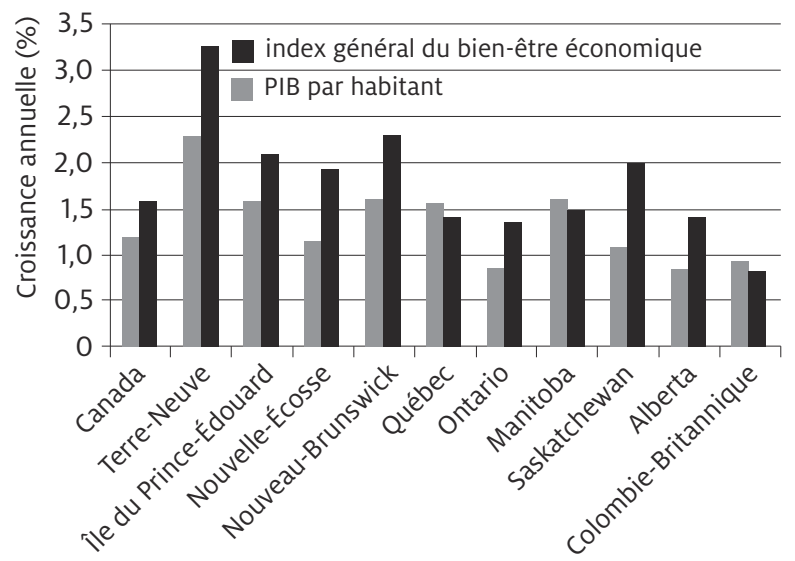

Source: Lars Osberg, Andrew Sharpe, «New Estimates of the Index of Economic Well-being for Canada and the Provinces, 1981-2008», CSLS Report, décembre 2009, p. 14.

GRAPHIQUE 6.3

L'évolution de l'IBEE et de ses composantes, Canada, 1981 et 2008

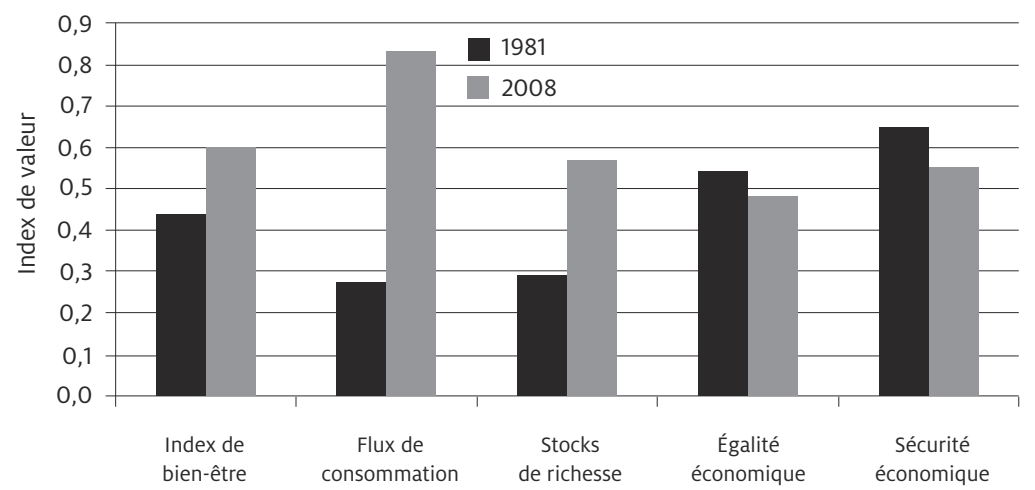

Source: Lars Osberg, Andrew Sharpe, «New Estimates of the Index of Economic Well-being for Canada and the Provinces, 1981-2008», CSLS Report, décembre 2009, p. 17. 
du PIB comme indicateur, d'identifier les compléments qui devraient lui être apportés et d'évaluer la pertinence d'indicateurs alternatifs. Le rapport, présenté en septembre 2009, se compose de trois parties ${ }^{13}$. La première revient sur le PIB et ses limites. La deuxième explore l'aspect multidimensionnel d'une mesure de la «qualité de la vie». La dernière met l'accent sur les questions environnementales et de soutenabilité des modèles de croissance.

La légitimité scientifique de la commission a souvent été soulignée par la participation de cinq prix Nobel d'économie (outre Amartya Sen et Joseph Stiglitz, on retrouvait les professeurs Kenneth Arrow, Daniel Kahneman et James Heckman). On comptait également parmi les collaborateurs du rapport les représentants des principaux instituts statistiques, comme l'Insee en France ou l'OCDE, longtemps hermétiques à toute critique du PIB comme étalon.

Pour les économistes hétérodoxes menant depuis plusieurs décennies la contestation du PIB comme étalon du «bien-être économique», c'est une reconnaissance spectaculaire de leurs thèses ${ }^{14}$, par des autorités pourtant plus conventionnelles. Ainsi, le rapport reconnaît la cécité que suscite le déchiffrement de l'économie et du social à la seule lumière du PIB et de sa croissance. L'augmentation de la production

13. Joseph Stiglitz, Amartya Sen, Jean-Paul Fitoussi, Rapport de la Commission sur la mesure des performances économiques et du progrès social, Ministère de l'Économie, de l'Industrie et de l'Emploi, 2009. www.stiglitz-sen-fitoussi. fr.

14. Voir les analyses du rapport sur le site du FAIR (Forum pour d'autres indicateurs de richesse), et notamment les critiques qu'ils y apportent: Institut pour le développement de l'information économique et sociale, FAIR, www.idies.org/index.php?category/FAIR. Ils critiquent notamment, au sein de ce rapport, la désarticulation des dimensions sociales et environnementales du développement durable, la monétarisation systématique du nonmarchand et du non-monétaire (même pour le valoriser), ainsi que le cloisonnement technocratique de la commission, cénacle d'économistes consacrés, sans échange organisé avec les organisations de la société civile. 
et des profits financiers n'est pas synonyme d'avancée systématique sur le plan social et sur le plan environnemental. Ces indicateurs hégémoniques ne nous renseignent ni sur le bien-être durable, ni sur les inégalités, ni sur la pression environnementale. A contrario, dans la lignée des travaux que nous avons présentés précédemment (mais dont l'écho politique fut bien moindre), le rapport met l'accent sur la question des inégalités et de l'insécurité économique et sociale; il évoque la nécessité de mieux valoriser (monétairement ou non) le non-marchand et le non-monétaire, les services publics et leurs contributions, le travail domestique et les loisirs. Il reconnaît aussi l'intérêt du concept de «dépenses défensives ».

Plus précisément, il appelle à mettre l'accent sur trois axes. Sur le plan économique, il propose d'apporter un ensemble de compléments et de correctifs au PIB. Sur un plan plus large de «bien-être», il valorise une évaluation tant subjective (ressentie par les ménages) qu'objective avec une prise en compte de facteurs multidimensionnels (conditions de vie matérielles, santé, éducation, participation à la vie politique, liens sociaux ${ }^{15}$, environnement, insécurité économique et physique). Contrairement aux productions statistiques habituelles, le rapport appelle à une mesure de la consommation plutôt que la production, à une prise en compte plus grande du poids du patrimoine, de la question de la répartition des revenus et des richesses, et, enfin, d'une évaluation conséquente du non-marchand pour mieux approcher le «revenu total» (full income) des ménages. Enfin, sur le plan de l'axe sur la soutenabilité du développement, le rapport invite, contrairement à la pratique statistique traditionnelle des comptabi-

15. Soulignons la participation de Robert Putnam parmi les contributeurs au rapport. 
lités nationales, à effectuer désormais non seulement des observations, mais des projections systématiques. Puis il émet deux recommandations. La première appelle, afin d'évaluer la soutenabilité, à la formalisation d'un tableau de bord spécifique, permettant de mesurer les variations des «stocks» de ressources nécessaires pour la préservation du bien-être futur (ressources naturelles mais aussi capital humain, social et physique). La seconde exige d'accorder un suivi séparé aux aspects environnementaux de la soutenabilité, ayant notamment trait à des biens publics mondiaux, comme le climat, à travers des indicateurs monétaires, mais aussi physiques.

Assiste-t-on au sein des réseaux de spécialistes et de gouvernants à un basculement de paradigme ou à un simple ravalement de façade de l'édifice du pouvoir, par le recours aux discours critiques d'un mode de développement socioéconomique soumis à des démentis cinglants en cette fin de décennie? Le sort qui sera réservé à ce rapport constituera sans doute un indicateur précieux pour l'évaluer. Seule certitude: sa consécration ou son enterrement ne reposera pas seulement sur des critères de validation scientifique. On a en effet démêlé précédemment les intrications complexes entre les dimensions politiques et scientifiques de la statistique. Or, on voudrait démontrer maintenant que les modalités contemporaines de la gouvernance, par la diffusion du recours à une technique comme le benchmarking, accentuent encore davantage la dimension instrumentale de l'objectivation statistique sur les questions de pauvreté et d'inégalités sociales. 


\section{L'objectivation statistique du social à l'épreuve du benchmarking politique}

En 2009 est paru en France un ouvrage intitulé Le grand trucage. Comment le gouvernement manipule les statistiques. Sa particularité est d'être signé par un pseudonyme (Lorraine Data), désignant «un collectif de fonctionnaires issus de la statistique et de la recherche publique, dont la plupart sont tenus à l'obligation de réserve». Loin d'une charge aveugle et générale, sur le mode de la «statistique comme art de mentir avec exactitude», les auteurs reviennent au contraire en détail sur quelques opérations précises permettant de «faire dire» aux statistiques un certain nombre de choses en appui d'une politique donnée. Les quatre techniques principales sont:

- ne retenir que ce qui arrange (parmi un ensemble d'indicateurs, choisir celui qui sert le mieux le propos ou bien retarder la publication des plus embarrassants);

- utiliser un indicateur «écran» (mettre l'accent sur une moyenne, par exemple, permet de masquer l'ampleur des inégalités);

- changer la façon de compter en gardant apparemment le même indicateur (un classique est l'affectation de demandeurs d'emploi à une nouvelle catégorie, permettant de faire baisser le taux de chômage);

- faire dire à un chiffre ce qu'il ne dit pas (par exemple «en confondant les statistiques relatives à l'activité de la police et de la gendarmerie avec une mesure du niveau effectif de la délinquance $\left.{ }^{16} »\right)$.

Les auteurs reviennent notamment sur l'application de ce type d'opérations dans un certain nombre de débats socioéconomiques récents (pouvoir d'achat, chiffres de l'emploi

16. Lorraine Data, Le grand trucage. Comment le gouvernement manipule les statistiques, La Découverte, p. 14. 
et du chômage et bien sûr mesure de la pauvreté) et dénoncent ce qu'ils qualifient de «manipulation des chiffres».

Certes, comme on l'a rappelé, l'intervention du pouvoir politique dans l'administration de la statistique n'est pas un phénomène nouveau. De même, on peut incriminer tel ou tel gouvernement comme plus susceptible de faire usage d'un contrôle «serré» de ces instruments. Mais, plus important, il nous semble que c'est la recomposition des modalités de gouvernement actuelles qui permet de mieux comprendre cette articulation particulière entre politique et statistique. En effet, depuis quelques années, le benchmarking (l'étalonnage des performances) a pris une importance particulière, notamment dans le cadre de la construction européenne ${ }^{17}$. Issu du management des entreprises privées, le benchmarking est une technique de gestion par objectifs. La quantification systématique des résultats, via des systèmes de tableau de bord et d'indicateurs, sert à rationaliser l'activité, à l'évaluer, mais aussi à susciter une émulation entre les opérateurs, par la publicité des performances de chacun. La coordination du collectif se fait donc sur un mode à la fois coopératif et compétitif, dit de «co-opétition». La surveillance systématique des performances chiffrées des uns et des autres doit conduire au repérage et à l'adoption des «meilleures pratiques». Cette technique issue du secteur marchand a été importée par le nouveau management public comme une méthode applicable aux administrations publiques.

Au sein de l'Union européenne, cette technique fut mise en place non seulement sous couvert de «bonne gouvernance»,

17. Pour des développements sur cette partie, on renvoie à la thèse d'Isabelle Bruno, «Déchiffrer l'“Europe compétitive”. Étude du benchmarking comme technique de coordination intergouvernementale dans le cadre de la stratégie de Lisbonne», Thèse de doctorat, Département de science politique, IEP de Paris, 2006. 
mais également comme stratégie de coordination intergouvernementale à la suite du Sommet de Lisbonne (23-24 mars 200O). Lors de ce sommet, l'Europe communautaire se fixe pour objectif de devenir en l'espace d'une décennie «l'économie de la connaissance la plus compétitive et la plus dynamique du monde, capable d'une croissance économique durable accompagnée d'une amélioration quantitative et qualitative de l'emploi et d'une plus grande cohésion sociale ${ }^{18}$ ». Or, on touche ici à des domaines où l'UE ne dispose pas de compétences législatives fortes, notamment dans le domaine social et de l'emploi (règle de la subsidiarité).

La «méthode ouverte de coordination» (MOC) initiée à la suite de ce sommet vise à formaliser une coopération intergouvernementale sur ces sujets sans dessaisir les États de la maîtrise de leurs politiques publiques respectives. Le benchmarking devient la pierre angulaire de cette coordination sur le double mode coopératif et compétitif indiqué précédemment. En effet, par le partage des informations et l'harmonisation des seuils et références nationales, on crée un espace de «commune mesure» permettant à la fois la concertation, mais aussi la comparaison et le classement des «bons» et «mauvais élèves». Ainsi, comme le note Isabelle Bruno, on passe d'une européanisation par le droit à une européanisation par le chiffre. Celle-ci prend la forme d'étalons à atteindre (par exemple, dans le domaine de l'emploi: un taux d'emploi de $70 \%$ pour la population considérée en âge actif (15-64 ans), 50\% pour les 55-64 ans et $60 \%$ pour les femmes) et d'indicateurs, à l'image des dix-huit indicateurs de pauvreté et d'exclusion sociale retenus par le Conseil européen à l'occasion de la réunion de Laeken en décembre

18. Conseil européen, «Conclusions de la Présidence», Sommet européen de Lisbonne, 23 et 24 mars 2000, Nr: 100/1/OO, \$5. 
2001. À l'occasion de la publication régulière des «palmarès» divers, les gouvernements nationaux subissent une pression vis-à-vis de l'opinion publique nationale, par jeu de comparaison avec leurs partenaires-concurrents. De plus, l'octroi de financements est corrélé aux résultats de ces différents palmarès.

Les opérations de qualification/disqualification régies par la discipline de l'étalonnage des performances font donc peser un poids stratégique sur la production des données statistiques, qui objectivent les «performances» de chacun. Comme l'écrit Boltanski à propos des «épreuves» de réalité qu'instaure le benchmarking, «la description [...] assume alors explicitement l'existence [d'une] boucle de rétroaction entre le sujet et l'objet de la connaissance, et les met en œuvre de manière stratégique pour accroître l'efficacité de mesures visant à modifier les contours de la réalité ${ }^{19}$ ».

La manière dont sont produites les statistiques est donc cruciale. Or, l'UE est dans une situation d'asymétrie d'informations vis-à-vis des données statistiques produites par les administrations nationales (et ce, spécifiquement dans le domaine social, où la DG Emploi a toujours été un service «faible $\left.»^{20}\right)$. Ainsi, le plus souvent, les données utilisées pour les indicateurs de performance proviennent de l'Office statistique des Communautés européennes (Eurostat). Mais cet organisme n'entreprend pas lui-même de collecte de données; il recueille et met en commun celles produites par les instituts nationaux. Eurostat détient un rôle clef, à la fois comme architecte du benchmarking, pour traduire les demandes politiques en statistiques et pour mener l'harmonisation

19. Luc Boltanski, De la critique. Précis de sociologie de l'émancipation, Gallimard, 2009, p. 208.

20. Cécile Robert, "L'impossible "modèle social européen"», Actes de la recherche en sciences sociales, $\mathrm{n}^{\text {os }}$ 166-167, mars 2007, p. 94-109. 
des statistiques nationales, et comme interface entre la DG Emploi et les États membres ${ }^{21}$. Cette tentative de rendre commensurable le social en Europe, c'est-à-dire à la fois quantifiable et comparable, est source de tensions politiques, qui prennent les atours de controverses scientifiques.

Le témoignage d'une administratrice de l'Insee, qui a contribué au sein d'Eurostat à un projet d'indicateur des «sans-abri», rend compte de ces tensions ${ }^{22}$. Elle montre les multiples controverses qui ont éclaté lors de la tentative de trouver un point d'accord sur la manière de définir et de compter les «sans-abri», à la fois entre secteur caritatif et État, et entre les différents États-membres. Ainsi, il s'est avéré impossible d'arriver à un consensus puisque la diversité des modalités nationales d'appréhension du phénomène interdisait un accord sur l'objet même de la politique. Ainsi, comment évaluer, au-delà d'une collection d'individus et de situations particulières, l'étendue d'un phénomène, dont la dénomination même pose problème? En effet, dans les pays de langue latine et slave, il n'existe pas de terme désignant de manière globale le phénomène, à la différence du vocable de «Homelessness» ou de «Wohnungslosigkeit». La diversité culturelle et institutionnelle sédimentée de manière séculaire dans les approches nationales eut raison de la volonté d'une «harmonisation» sur le plan communautaire.

Cette difficulté à opérer une harmonisation systématique laisse donc une marge de manœuvre aux instituts statistiques nationaux. Pour Robert Salais, ceux-ci se trouvent pris au milieu d'un jeu entre plusieurs acteurs, au sein de la stratégie européenne pour l'emploi (SEE) initiée au Sommet

21. Delphine Nivière, «Négocier une statistique européenne: le cas de la pauvreté», Genèses, vol. 58, mars 2005, p. 28-47.

22. Cécile Brousse, «Définir et compter les sans-abri en Europe: enjeux et controverses», Genèses, vol. 58, mars 2005, p. 48-71. 
de Lisbonne, à travers la MOC. Il pointe un risque de «dérive instrumentale» puisque «l'étalonnage par les indicateurs sert à piloter la SEE et non à évaluer économiquement ses résultats. [...] La coopération consiste, pour chaque État membre, à infléchir pour son propre compte les règles d'éligibilité de ses propres mesures, ainsi que leur mise en œuvre par les agences publiques, pour qu'elles entrent dans le cadre des indicateurs européens ${ }^{23}$.» On observe ainsi un jeu misavant mi-politique d'opérations de traduction et de conversion, à travers la quantification et les indicateurs, qui permet, au sein d'un apparent espace de «commune mesure» où chacun semble parler le même langage, la persistance de stratégies et d'héritages institutionnels distincts ${ }^{24}$.

Derrière ce brouillage des repères opéré par l'instrumentation et l'instrumentalisation statistique, il est difficile d'apercevoir avec clarté ce qu'il en est de la supposée convergence ou de l'irréductible divergence des modèles socioéconomiques. Notre étude croisée des configurations danoise, britannique, française et québécoise offre plusieurs clefs pour instruire ce débat. Elle nous permet de nous détacher des faux-semblants sur lesquels se porte trop souvent une perspective comparative (indicateurs affichés, mesures et dispositifs annoncés), pour appréhender l'inscription de

23. Robert Salais, «La politique des indicateurs. Du taux de chômage au taux d'emploi dans la stratégie européenne pour l'emploi», Bénédicte Zimmermann (dir.), Les sciences sociales à l'épreuve de l'action. Le savant, le politique et l'Europe, Éditions de la Maison des sciences de l'homme, 2004, p. 315.

24. Isabelle Bruno rapporte, à la suite de la publication de chiffres relatifs à l'effort en recherche et développement de 18 pays, l'effort de la Commission européenne pour ménager les susceptibilités de chaque pays: «Ils ont illustré les quinze indicateurs par 39 diagrammes de telle sorte que chaque État membre puisse se vanter de figurer en tête d'un classement», Isabelle Bruno, «Y a-t-il un pilote dans l'Union? Tableaux de bord, indicateurs, cibles chiffrées: les balises de la décision», Politix - Revue des sciences sociales du politique, vol. 82, $\mathrm{n}^{\circ} 2,2008$. 
formes différenciées de la pauvreté dans des architectures sociales et politiques sédimentées. C'est à cette condition qu'on peut restituer le sens des différentes mesures prises au niveau national pour s'accommoder de la pauvreté, comme le dernier chapitre s'y attelle. 


\section{CHAPITRE 7}

\section{Vous dites convergence?}

Les études, particulièrement celles qui nous viennent d'Europe, ont été largement influencées par le contexte de la construction européenne où la convergence a clairement été identifiée comme un objectif technocratique à atteindre. D'un point de vue analytique, cette injonction à la convergence a conduit les chercheurs à construire différentes mesures. Essentiellement, les politiques et les programmes mis en place ont servi d'unité de mesure. Dans le champ social, deux grandes questions traversent les travaux: est-ce que les politiques sociales des États sont plus semblables aujourd'hui qu'hier? Est-ce que les orientations des politiques vont dans la même direction?

\section{Une convergence qui se remarque}

Plusieurs réponses se côtoient. À un certain niveau de généralité, les travaux ont noté un accroissement de la convergence de l'intervention publique, au moins dans l'esprit des réformes mises en place. Partout au sein de l'Union européenne, la question sociale est devenue une question de politique de ressources humaines. La notion «d'investissement social»a 
été proposée pour cerner les changements survenus dans la façon d'aborder la question sociale ${ }^{1}$. Dans la perspective de l'investissement social, l'État social a regagné ses lettres de noblesse dans la mesure où certaines interventions ciblées sont mises de l'avant pour répondre aux nouveaux risques sociaux et économiques. La responsabilité du «bien-être» redevient partagée entre les citoyens, l'État et la solidarité de proximité. Il ne s'agit pourtant pas d'un simple retour au keynésianisme, puisque la «perspective macroéconomique de l'investissement social est, tout comme l'était celle des néolibéraux, axée sur l'offre ${ }^{2} »$. Autrement dit, les États européens, devant les impasses sociales et politiques produites par deux décennies de politiques néolibérales, ont accepté l'idée que l'intervention publique dans le domaine social pouvait aussi être productive et servir le développement économique. Plusieurs réformes des politiques de formation professionnelle ou de pensions et des mesures destinées aux enfants illustrent ces changements. Dans le champ de la pauvreté et des inégalités, la perspective de l'investissement social s'est accompagnée d'un intérêt pour la cohésion sociale et son pendant, l'exclusion. Avec la relance de la stratégie de Lisbonne en 2005, l'exclusion sociale est acceptée comme un concept multidimensionnel et l'accent est mis sur la nécessité de «l'inclusion active» et de la lutte contre la pauvreté des enfants ${ }^{3}$.

1. Jane Jenson, «Redesigning Citizenship Regimes after Neoliberalism. Moving Towards Social Investment», Nathalie Morel, Bruno Palier, Joakim Palme (dir.), What Future for Social Investment?, Institute for Futures Studies Research Report, 2009, p. 27-44.

2. Ibid., p. 30.

3. Kerstin Jacobsson, «Achievements and Non-Achievements of the European Employment Strategy», Nathalie Morel, Bruno Palier, Joakim Palme (dir.), What Future for Social Investment?, Institute for Futures Studies, Research Report, 2009, p. 119-130. 


\section{Dissonances ou affirmation de modèles}

Cette convergence dans la représentation et la justification de l'intervention de l'État ne signifie pas pour autant une convergence équivalente dans le contenu même des politiques. Le concept s'accompagne plutôt d'une diversité d'interprétations et de mises en pratique ${ }^{4}$. Plusieurs travaux récents ont essayé de mesurer l'investissement social dans certains États afin de statuer sur le changement ainsi que sur la question de la convergence.

Lorsque l'on compare les dépenses sociales allouées à des politiques dites «d'investissements» — «les politiques qui alignent leur champ de dépenses sur des provisions pour le futur et pour les besoins des jeunes générations»- et les politiques dites «compensatoires» - les «compensations généreuses de risques sociaux comme le chômage, la vieillesse et les mesures passives du marché du travail ${ }^{5} »-$, on peut retenir quelques observations. Il y a une plus grande similarité entre les pays depuis 1980 du point de vue du total des dépenses sociales qui ont augmenté (l'écart entre les pays s'étant aussi réduit), mais, pour autant, il n'y a pas de convergence des pays sur les ressources consenties aux deux types de mesures. Trois catégories de pays se dégagent: les pays d'Europe du Nord où les deux types de dépenses sont élevés; les pays anglophones, la Norvège et la Suisse, où existe un faible niveau de dépense pour les deux types de

4. Alexandra Dobrowolsky, Denis Saint-Martin, «Agency, Actors and Change in a Child Focused Future: "Path Dependency" Problematised», Journal of Commonwealth and Comparative Politics, vol. 4, n ${ }^{\circ} 1,2005$, p. 1-33; Jane Jenson, "Children, new social risks and policy change. A LEGO ${ }^{\mathrm{TM}}$ future?», Comparative Social Research, vol. 25, 2008, p. 357-381.

5. Rita Nikolai, «Towards Social Investment? Patterns of Public Policy in the OECD World», Nathalie Morel, Bruno Palier, Joakim Palme (dir.), What Future for Social Investment?, Institute for Futures Studies Research Report, 2O09, p. 101-1O2. 
mesure; les pays d'Europe continentale, d'Europe du Sud et le Japon, où les dépenses compensatoires demeurent élevées alors que les dépenses d'investissement social restent faibles.

Dans la même veine, l'analyse des variations dans la mise en pratique de l'investissement social, qui tient compte d'une cinquantaine d'indicateurs, permet d'identifier trois modèles d'investissement social ${ }^{6}$. Le premier modèle qui correspond grosso modo aux pays sociaux-démocrates, est qualifié de «high social investment activation». Il allie bonne santé des populations, niveau élevé d'éducation et forte participation au marché du travail sur la longue période. Le deuxième, le modèle qualifié de «low social investment activation», combine un taux élevé de participation au marché du travail et des formes privées de sécurité sociale et d'éducation qui ne profitent pas à tous. Une partie des travailleurs sont moins qualifiés et en moins bonne santé, si bien que l'investissement social concerne d'abord les populations riches. Ce modèle est caractéristique des États libéraux. Le troisième modèle n'est pas centré sur l'investissement social, mais offre des prestations passives et généreuses «à des travailleurs qui se retirent tôt dans leur vie du marché du travail», créant un déséquilibre et menaçant la capacité financière du régime à cause d'un taux très bas de participation au marché du travail ${ }^{7}$. Ce modèle, assez éloigné de la perspective d'investissement social, décrit assez bien les pays d'Europe continentale.

L'analyse de la nature des dépenses sociales en Europe et leur variation permet également d'estimer le phénomène de

6. Paul Bernard, Guillaume Boucher, «Institutional Competitiveness, Social Investment and Welfare Regimes », Regulation \& Governance, vol. 1, n 3, 2007, p. 213-229.

7. Ibid., p. 224. 
la convergence ${ }^{8}$. En se référant aux quatre catégories d'État social (nordique, continental, anglo-saxon et méditerranéen), il ressort que la variation des dépenses sociales à l'intérieur d'une même catégorie de pays a augmenté entre 1992 et 2002, alors qu'elle a diminué entre les différents groupes. Par ailleurs, la convergence est faible, si on compare la croissance du volume de dépenses sociales entre 1981 à 1991 et 1991 à 2001. La prise en compte, non plus seulement de l'évolution des dépenses sociales des États, mais également des revenus, de la générosité des prestations et du niveau de démarchandisation des politiques sociales, met en évidence qu'il n'y a pas de «course vers le bas» ni de convergence vers le modèle américain, mais plutôt des mouvements de rattrapage entre les pays qui expliquent, en grande partie, la diminution des écarts entre les pays au chapitre des dépenses sociales totales, des dépenses sociales, pour prestation versées en espèces ou en services, des dépenses en santé et pour la protection contre le chômage ${ }^{9}$.

Néanmoins, on comprend que les modes d'intervention des États restent distincts. L'Union européenne a certes construit de la convergence symbolique et empirique en diffusant des discours et des indicateurs de convergence, mais les analyses statistiques ne permettent pas de conclure à une convergence des politiques elles-mêmes. Il n'y aurait donc rien de mécanique dans les évolutions des États sociaux: ni mouvement structurel dû à la mondialisation, ni course vers le bas (la thèse de l'américanisation) ou vers le haut (la thèse de l'européanisation), ni recherche des «meilleures»

8. Tito Boeri, Simona Baldi, «Europe(s) sociales, convergence et compétition», Revue d'économie politique, vol. 115, 2005/2006, p. 170-171.

9. Peter Starke, Herbert Obinger, Francis G. Catles, «Convergence Towards Where: In What Ways, if any, Are Welfare States Becoming More Similar?», Journal of European Public Policy, vol. 15, nº 7, 2008. 
pratiques (la thèse des décideurs comme acteurs rationnels), mais tout au plus des modalités d'apprentissage entre les sociétés qui peuvent expliquer certaines réformes de politiques ou de programmes et leur diffusion dans le temps et l'espace $^{10}$.

\section{Différenciation et complexité sociales}

Cette réponse, pour intéressante qu'elle soit, est insatisfaisante. Les dimensions de la convergence ne se limitent pas à une question de nature technique des politiques, ni aux seules sommes allouées à chaque programme. D'ailleurs, la pauvreté et les inégalités sociales ne sont pas uniquement du ressort de l'action publique. Ainsi, au-delà de l'instrumentalisation des politiques, nous devons nous demander si les sociétés convergent. Plus précisément, est-ce que les formes de pauvreté convergent au sein de ces sociétés?

Dans cet ouvrage, la question de la pauvreté n'a pas été posée d'abord en termes d'instrumentation de l'intervention sociale, mais davantage en termes de configuration sociétale et de physionomie de l'action publique qui supportent la constitution d'une pauvreté plus ou moins importante, la perpétuation plus ou moins poussée de cette situation, du reflux d'une population plus ou moins considérable en marge du modèle de développement, de l'investissement public relatif dans le champ de la redistribution de la richesse, de l'intensité de l'accès au travail, de la capacité socioécono-

10. Diane Stone, «Transfer Agents and Global Networks in the Transnationalisation of Policy», Journal of European Public Policy, vol. 11, nº 3, 2004, p. 545-566; Gérard Boismenu, Pascale Dufour, «Participation de l'État et de l'environnement social à l'aide aux personnes sans emploi. Une comparaison des aides publiques aux États-Unis, Canada, Grande-Bretagne, Danemark et France», Recherches et Prévisions, $\mathrm{n}^{\circ}$ 91, mars 2008; Gérard Boismenu, Pascale Dufour, Alain Noël, «Les logiques de la contrepartie dans les programmes visant les personnes sans emploi», Les Politiques sociales, 2004, p. 106-117. 
mique et de la qualité d'intégration des populations travailleuses dans la sphère du travail. En somme, il nous importait de comprendre la structure des pratiques et les formes institutionnelles publiques et privées qui produisent et supportent la pauvreté, ainsi que l'inégalité sociale.

Ces considérations sont éclairées par une série de relations dans des sociétés comparables pour leur type de développement économique et pour leurs traditions politiques, pris en termes généraux. Malgré la compatibilité d'ensemble de ces sociétés, nous avons constaté que si la pauvreté et l'inégalité sociale sont des réalités qui habitent chacune d'elles, ces phénomènes prennent une ampleur, des accents et des formes d'existence contrastés. Des «modèles» existent et ceux-ci représentent autant de constructions sociales et politiques qui ont marqué l'histoire de ces sociétés. Ces modèles ne sont rien d'autre que des agencements de pratiques et de formes institutionnelles qui traversent la sphère du travail, les modes de représentation des intérêts et les logiques de politiques publiques. Avec le recul et la vision panoramique que ce recul permet, on remarque qu'il y a une compatibilité et une complémentarité dans l'empilement des formes de pratiques, des institutions et des arbitrages. Mais, loin de relever d'une préscience et d'une volonté historique intangible, il s'agit là d'une compatibilité qui exprime une représentation inscrite dans des logiques d'action et d'intervention des acteurs privés et publics.

Lorsqu'on discute de la situation d'une société, on ne peut échapper au fait que cette société est inscrite et participe à un modèle particulier d'organisation sociale et qu'un changement de «parcours» ou un changement institutionnel ne peut se faire que dans des contraintes de tous ordres et sur lesquelles il faut agir, tout en sachant que le seul volontarisme politique a de grandes limites. 


\section{L'innovation plutôt que la fatalité}

La comparaison internationale permet de distinguer ces modèles et cela est instructif à plusieurs titres.

- On comprend que l'on ne peut parler en termes de modèles que dans la mesure où on procède à une généralisation empirique, basée sur l'observation de modes de fonctionnement, d'organisations des pratiques, d'architectures institutionnelles, de modèles de développement, de rapports à la population, de cultures politiques, etc., permettant d'établir des congruences.

- Les sociétés qui sont associées à tel ou tel modèle ne le sont pas en fonction d'une fatalité historique et n'existent pas en fonction de l'un ou l'autre des modèles. Une société associée à un modèle - sachant que chaque société est singulière et que son association à un modèle relève d'une stylisation dans la lecture de la complexité sociale - peut l'être dans le temps, sans que cela préjuge du parcours ou du cheminement suivi dans le cours de l'évolution des choses. Une société n'a pas pour mission d'«incarner un modèle»!

- Il reste que les similarités repérables pour des modes de représentation des acteurs, des formes institutionnelles et des logiques d'action exercent un poids sur cette évolution, du moins en termes de contraintes. Cette remarque est d'autant plus pertinente dans la perspective où l'on voudrait s'inspirer de façons de faire empruntées à d'autres contextes: le mimétisme gouvernemental ou l'apprentissage social pour les politiques publiques, par exemple, qui ne tiendrait pas compte de leurs conditions d'existence et des contraintes inhérentes constituerait une méconnaissance pouvant hypothéquer toute l'entreprise.

À partir des travaux existants, on constate sans peine que des modèles différenciés émergent sur le front de l'inégalité 
des revenus. La disparité dans la distribution sociale de la richesse prend des accents très poussés dans les pays de tradition libérale, alors que, malgré un accroissement des écarts, cela reste dans des ordres de grandeur nettement plus restreints dans les pays sociaux-démocrates, par exemple. Même si cela nécessite beaucoup de nuances, le démarquage qui ressort avec les trois modèles - d'économie libérale de marché, d'économie sociale de marché continentale ou d'économie sociale de marché nordique - propose un cadrage assez instructif des modèles d'économie politique et de formes institutionnelles. L'inégalité n’a rien d'un épiphénomène: elle tire sa source de l'accès au travail, des structures salariales, du statut au travail, des rapports de force sur la scène du travail et en dehors, de la hiérarchisation des compétences professionnelles, de la place relative du secteur public et du poids des institutions publiques dans la distribution de la richesse. L'approfondissement des inégalités n'est pas un accident historique: il est soutenu par une organisation sociale puissante qui est compatible et permissive pour une plus ou moins grande disparité.

Dans la littérature, le traitement de la pauvreté met en relief les questions de l'accès à l'emploi, du statut d'emploi, de la composition des ménages (famille monoparentale, famille avec un ou deux revenus) et le rôle (et l'ampleur) de l'intervention publique, tout autant pour l'accès à l'emploi que pour la résorption du phénomène de pauvreté en tant que tel. Dans cette perspective, les pouvoirs publics ne sont pas un intervenant externe et ponctuel à l'égard d'un modèle de développement, mais bien un élément interne à l'œuvre dans divers champs de pratiques, comme partie constitutive de ces pratiques elles-mêmes. On a pu montrer, pour prendre un exemple particulier, que l'État social dans la régulation des populations prenait des physionomies distinctes selon 
que l'on favorisait les services plutôt que les transferts, les examens de ressources plutôt que l'universalité, la citoyenneté sociale plutôt que l'assurance sociale ${ }^{11}$. De nombreuses études ont montré que ces physionomies différentes étaient liées (comme facteurs explicatifs) aux risques sociaux, aux clivages politiques et aux institutions politiques ${ }^{12}$.

Autre exemple: les politiques d'activation se sont généralisées dans l'arsenal des dispositifs mis en œuvre pour remodeler les conditions de soutien au revenu et pour favoriser l'accès au travail. Mais elles suivent des logiques qui sont fortement contrastées. En grossissant le trait, deux approches qui reposent sur les postulats et des méthodes différentes, voire opposées, se mettent en œuvre ${ }^{13}$. D'un côté le work first, où la dépendance à l'aide publique est combattue au moyen d'obligations, d'incitation forte, de démarche ciblée et d'une vision punitive au besoin, pour inciter un engagement des sans-emploi sur le marché du travail. Les dispositifs combinent les minima sociaux et l'impôt négatif, mais ne portent pas ombrage au développement du «travail pauvre» et se concilient bien avec des formations peu qualifiantes. D’un

11. Lyle Scruggs, «The generosity of social insurance, 1971-2002», Oxford Review of Economic Policy, vol. 22, $\mathrm{n}^{\circ}$ 3, 2006.

12. Torben Iversen, David Soskice, «Electoral Institutions and the Politics of Coalitions: Why Some Democracies Redistribute More than Others ", American Political Science Review, vol. 100, n² 2, 2006, p. 165-181; Alberto Alesina, Edward L. Glaeser, Combattre les inégalités et la pauvreté. Les États-Unis face à l'Europe, Flammarion, 2004; Torben Iversen, John D. Stephens, «Partisan Politics, the Welfare State, and Three Worlds of Human Capital Formation», Comparative Political Studies, vol. 41, $\mathrm{n}^{\text {os }}$ 4/5, 2008, p. 600-637.

13. Jean-Claude Barbier, «Peut-on parler d'«activation» de la protection sociale en Europe?», Revue française de sociologie, vol. 43, $\mathrm{n}^{\circ}$ 2, 2002; Ides Nicaise, «The active welfare state: a response to social exclusion?», Vives lectures, 2002; Yannick L'Horty, Revenu minimum et retour à l'emploi: une perspective européenne?, Université d'Evry, 2004; Pascale Dufour, Gérard Boismenu, Alain Noël, L'aide au conditionnel: la contrepartie dans les mesures envers les personnes sans emploi en Europe et en Amérique du Nord, Presses de l’Université de Montréal, 2003. 
autre côté l'inclusion sociale, où il faut chercher à atteindre la participation sociale par l'emploi, ce qui passe par l'employabilité et la formation professionnelle, soit l'intégration par l'emploi. On préconise une protection de bon niveau et prolongée, basée sur des droits, mais également des obligations, se conciliant à des droits et à des sanctions moins rigoureuses. Ces deux démarches correspondent assez bien au modèle d'économie libérale de marché et au modèle de l'économie sociale nordique. Mais dans tous les cas, cette tension bipolaire aiguise l'évolution des dispositifs de l'activation.

Ces quelques observations illustrent le caractère non aléatoire des orientations générales des politiques publiques à moyen terme. Parler de non-aléatoire ne signifie pas que l'on puisse avancer que ces politiques soient prévisibles. La comparaison que nous avons effectuée de deux sociétés «exemplaires» montre tout de même une transformation et un mouvement sinueux dans une trajectoire qui a ses propres forces d'inertie et qui connaît relativement peu de bifurcation radicale. Ainsi, le catalogue d'idées, la thématisation des enjeux, la configuration et la capacité organisationnelle des forces en présence, sans oublier le panorama de la structure industrielle et de ses acteurs majeurs, ont tendance à s'inscrire dans la durée. Bien souvent, il faut faire remonter le cadre des actions engagées à des moments charnières, qui sont relativement peu nombreux dans le parcours d'une nation. Mais aucune société n'est esclave de son passé ni n'est destinée à creuser le même sillon ou la même ornière.

\section{Une démarche multidimensionnelle}

Dans cette étude, nous avons adopté une démarche qui met en relation quatre dimensions de structuration de la pauvreté 
et des inégalités. La pyramide qui se révèle par l'articulation de ces dimensions découpe les formes multiples de pauvreté et d'inégalité ainsi que les dynamiques sociales, économiques et politiques de chacune des sociétés considérées.

La première dimension concerne les dynamiques en cours sur le marché du travail. L'analyse des systèmes de production est l'une des clefs de la compréhension de la pauvreté. Ces dynamiques économiques «brutes» sont aussi médiatisées par l'architecture des compromis sociaux institutionnalisés et des représentations sociales. Par exemple, dans certaines sociétés, la tolérance sociale aux inégalités, fruit de la construction historique des compromis sociaux et donc des luttes sociales et de leur plus ou moins grande reconnaissance par l'État, est plus faible que dans d'autres et se traduit par des législations du travail spécifiques, des niveaux de salaires minimum, des couvertures des risques sociaux plus ou moins étendues. Les dynamiques influant sur les formes de pauvreté sont aussi étatiques, dans la mesure où le traitement politique de la pauvreté par le biais de la gestion du chômage, du sous-emploi, des politiques d'assistance, d'activation, des politiques de formation et d'éducation, des politiques familiales parfois, a un effet direct sur la nature et les niveaux de cette pauvreté. Les dimensions symboliques qui entourent la notion de pauvreté sont également cruciales. Une société où la représentation dominante des personnes pauvres insiste sur la dimension de la responsabilité individuelle dans la situation de pauvreté n'induira pas les mêmes comportements, ni des décideurs ni des résidents, que celle où la responsabilité collective vis-à-vis de la pauvreté est dominante.

En considérant simultanément ces quatre dimensions, il est possible de saisir l'architecture générale des principaux processus qui sous-tendent le constat des formes multiples (et persistantes) de la pauvreté. Cette grille à quatre entrées 
ne prétend évidemment pas épuiser le sujet. Elle a néanmoins le mérite de permettre une comparaison relativement systématique de plusieurs configurations nationales différenciées.

Notre grille d'analyse permet d'apporter une première réponse générale à la question de la convergence/divergence des sociétés. Dans les chapitres précédents, nous avons montré que toutes les sociétés s'accommodent de formes particulières de pauvreté et d'inégalités. Néanmoins, pour reprendre de manière plus fine la comparaison, il est nécessaire de distinguer des tendances de fond, qui traversent l'ensemble des cas, ainsi que des articulations distinctes dans chaque société, qui font varier la valeur de l'accommodement de la pauvreté et des inégalités.

\section{Des tendances de fond}

Les deux dernières décennies ont généralement été marquées par une période de croissance économique importante et de recul du chômage. Seule la France contraste avec la persistance d'un taux de chômage relativement élevé. Cette période faste sur le plan des indicateurs économiques n’a pas entraîné une réduction significative de la pauvreté, ni des inégalités. Au mieux, les taux de pauvreté ont légèrement décru ou ils se sont stabilisés. Parallèlement, partout les inégalités se creusent. Les politiques d'activation, tant vantées par l'Union européenne et la troisième voie de Tony Blair ne se sont pas traduites par des résultats convaincants au chapitre de la pauvreté et des inégalités ${ }^{14}$. La perspective de l'investissement social a freiné le processus de retrait de

14. Jean-Claude Barbier, «Le workfare et l'activation de la protection sociale, vingt ans après: beaucoup de bruit pour rien? Contribution à un bilan qui reste à faire», Lien social et Politiques, vol. 61, 2009, p. 23-36. 
l'État engagé par les perspectives néolibérales de l'économie dans les années 1980, ce qui a permis de sauver l'essentiel: on est loin de la réforme sociale annoncée.

Le bilan n'est pas globalement satisfaisant, surtout si on met en lumière certaines manifestations que l'on peut cristalliser sous le vocable de précariat. Dans presque chacune des sociétés étudiées, un précariat s'affirme et s'institutionnalise ${ }^{15}$. Plusieurs éléments entrent en ligne de compte.

La généralisation des «travailleurs pauvres» — c'est-àdire des personnes qui exercent un emploi mais qui n'en retirent pas un salaire suffisant pour subvenir à leurs besoins de base -, participe au phénomène ${ }^{16}$. Réalité bien connue en Amérique du Nord, et en particulier aux ÉtatsUnis, l'existence des travailleurs pauvres est plus récente sur le continent européen (ou tout au moins sa reconnaissance est plus récente). Le travailleur pauvre se définit par son insuffisance de revenu, mais également par un statut d'emploi différent attaché à des droits sociaux soit distincts, soit inexistants. Dans les pays d'Europe continentale, comme la France ou l'Allemagne, la protection sociale du travailleur est directement liée au statut d'emploi. Si le statut d'emploi se dégrade, la protection sociale et les droits sociaux qui y sont attachés se dégradent également. D’où le développement d'un précariat qui se caractérise par des situations d'emplois non standard, mal payées et n'ouvrant pas des droits à l'assurance-chômage. Ce processus est valable ailleurs qu'en

15. Robert Castel, «Au-delà du salariat ou en deçà de l'emploi? L'institutionnalisation du précariat», Serge Paugam (dir.), Repenser la solidarité. L'apport des sciences sociales, PUF, 2007, p. 415-434.

16. Pierre-Joseph Ulysse, «Les travailleurs pauvres: de la précarité à la pauvreté en emploi. Un état des lieux au Canada/Québec, aux États-Unis et en France», Lien social et Politiques, vol. 61, 2009, p. 81-96. 
France ${ }^{17}$. Au Canada, par exemple, tout comme dans les régimes bismarckiens, une partie de la protection des travailleurs est encore associée au statut de travail et au nombre d'heures travaillées, en particulier pour l'assurance-chômage. Être en dehors du statut «normal» de travail à temps plein toute l'année, c'est être en dehors d'une partie de la protection sociale. Le précariat devient «une grille de lecture des évolutions de la société contemporaine ${ }^{18}$ ».

En France, le précariat économique, lié aux évolutions du marché du travail, est amplifié par «la gestion publique du chômage» qui a créé des statuts intermédiaires (préretraités, formation professionnelle des jeunes, emplois non marchands, emplois aidés), enchâssant de plus en plus dans des programmes des situations de travailleur de seconde zone $^{19}$. Au Québec, comme dans le reste du Canada, des droits différenciés en fonction de la nature de l'emploi existent, opposant en particulier les employés (bénéficiant de certains droits sociaux) aux travailleurs autonomes (non couverts). Plusieurs initiatives récentes des gouvernements du Québec ont conduit à mettre en place des programmes spécifiques pour les travailleurs autonomes comme les congés parentaux et l'assurance-chômage. On est loin cependant du traitement équivalent pour les deux catégories de travailleurs, alors que de plus en plus de personnes sont poussées vers le travail autonome, par la sous-traitance mais

17. Leah Vosko, Precarious Employment. Understanding Labour Market Insecurity in Canada, McGill-Queen's University Press, 2006; Rosalind Gill, Andy Pratt, «Precarity and Cultural Work. In the Social Factory? Immaterial Labour, Precariousness and Cultural Work», Theory, Culture and Society, vol. $25, \mathrm{n}^{\text {os }} 7-8,2008$, p. $1-30$.

18. Pierre-Joseph Ulysse, «Les travailleurs pauvres: de la précarité à la pauvreté en emploi. Un état des lieux au Canada/Québec, aux États-Unis et en France», Lien social et Politiques, vol. 61, 2009, p. 81-96.

19. Serge Paugam, Claude Martin, «Présentation. La nouvelle figure du travailleur précaire assisté», Lien social et Politiques, vol. 61, 2009. 
aussi par certains programmes d'activation qui valorisent le travail indépendant des prestataires. Là encore, le précariat devient un phénomène qui se répand et s'institutionnalise.

L'institutionnalisation du précariat peut prendre plusieurs formes. En France, le nouveau revenu social d'activité (RSA) crée un véritable statut de «travailleur précaire assisté ${ }^{20}$ » alors qu'au Québec, c'est le «travailleur autonome» qui devient de plus en plus un statut pour les travailleurs précaires. La reconnaissance formelle de ces statuts «entérine la distribution inéquitable des salaires parmi les travailleurs ${ }^{21} »$ et l'officialise. Au Danemark, la conjonction d'un haut niveau de protection sociale avec un haut niveau de flexibilité devrait empêcher l'apparition des travailleurs précaires, dans la mesure où les personnes en mobilité d'emploi ou de formation bénéficient d'une couverture sociale conséquente qui leur permet de se projeter dans l'avenir; il s'agit là d'une capacité essentielle qui fait défaut au travailleur précaire, dont le leitmotiv est l'instabilité. Ce système vertueux ne fonctionne que quand la mobilité sociale est réelle et partagée. Or, dans le cas danois, certaines catégories sociales, en particulier les personnes immigrantes, ne font pas partie de ces flux de main-d'œuvre, mais, au contraire, sont plus souvent à la marge du système. Il y aurait là tous les ingrédients d'une forme de précariat en développement. En GrandeBretagne, le précariat est clairement lié au phénomène des travailleurs pauvres, mais semble moins construit comme une strate sociale et plus comme un temps de passage vers le bas ou vers le haut. C'est un espace social où on séjourne, dans lequel on revient souvent, entre deux emplois stables

2O. Ibid., p. 15.

21. Pierre-Joseph Ulysse, «Les travailleurs pauvres: de la précarité à la pauvreté en emploi. Un état des lieux au Canada/Québec, aux États-Unis et en France», op. cit. 
ou deux périodes de chômage ou de formation, mais dont on n'est pas forcément prisonnier (comme en France).

Le précariat est un phénomène qui se généralise, même si la notion n'est pas présente partout. Relativement commun aux sociétés que nous avons comparées (sauf au Danemark), il semble durablement installé, autant comme mode d'adaptation des économies que comme catégorie d'action publique. Tout se passe comme si le précariat remplaçait le prolétariat de l'ère fordiste ${ }^{22}$. Sans en faire une classe sociale en tant que telle, nous pouvons considérer au moins le précariat comme un phénomène qui nous renseigne sur la «structure sociale inégalitaire, alimentée par des rapports sociaux discriminants ${ }^{23} »$. Cette structure sociale inégalitaire va varier suivant les sociétés et suivant les moments considérés: elle sera plus ou moins inégalitaire, la discrimination sera orientée vers telle ou telle catégorie sociale.

Outre le précariat, notre analyse met également en évidence l'existence d'un groupe de personnes «hors jeu» qui sont durablement exclues des modes de production et de consommation des sociétés. Chômeurs de longue durée, personnes itinérantes, personnes avec des problèmes durables de santé mentale et/ou physique, toutes ces personnes ne peuvent (et/ou ne veulent) se soumettre à l'injonction de «l'activation» et sont fréquemment l'objet de culpabilisation et de stigmatisation parce qu'elles ne travaillent pas. Cette dernière décennie, dans la perspective de l'investissement social, l'intervention publique a eu tendance à se détourner de ces populations considérées comme de mauvais investissements parce que non rentables dans le futur. C'est comme

22. Ibid., p. 83 .

23. Myriam Thirot, "Se brûler au travail": la précarisation du travail vécue par des habitants du Centre-Sud de Montréal», Lien social et Politiques, vol. 61, 2009, p. 161. 
si, pour elles, les dés étaient jetés. Évidemment, suivant les sociétés, le nombre et la forme spécifique de cette catégorie de personnes «hors jeu» varient, mais le processus de dualisation apparaît comme un élément relativement commun.

Précariat et personnes «hors jeu» sont des formes communes de pauvreté et révèle des inégalités partout présentes. Cependant, des articulations distinctes dans chaque société entre les quatre dimensions de notre grille d'analyse font que si toutes les sociétés s'arrangent d'un certain degré de pauvreté et d'inégalité, ce degré varie amplement, d'où l'intérêt de se pencher sur la logique générale de ces articulations.

\section{La logique des articulations}

À partir de la seconde moitié des années 1990, le Danemark est devenu le pays de l'Union européenne ayant le taux d'emploi le plus élevé, le taux de pauvreté et le niveau d'inégalité des revenus les plus bas.

Cette position au tableau d'honneur est fondée sur l'idée de la nécessité de la flexibilité de la main-d'œuvre, permettant aux employeurs d'ajuster leurs besoins rapidement. Les travailleurs danois sont en mouvement constant; s'ils connaissent des périodes fréquentes de chômage, elles sont de courte durée et la formation fait partie intégrante de leurs parcours de vie. En contrepartie, le niveau de sécurité sociale est élevé en termes de montant des revenus de remplacement, généreux en termes de durée et qualifiant dans la mesure où l'accent est mis sur l'activation, comme moyen d'enrichissement du «capital humain», et donc sur la formation qualifiante.

La gestion des politiques du marché du travail est tripartite, l'État, les syndicats et les représentants patronaux négocient de manière décentralisée la flexibilité des travailleurs 
ainsi que les salaires et les niveaux de protection sociale. La fiscalité très progressive limite les inégalités de revenus, de même que les programmes sociaux qui contribuent à la redistribution des richesses. Il n'y a pas de précariat en tant que tel au Danemark, néanmoins, nous avons noté des inégalités dans les trajectoires des travailleurs. Les travailleurs peu qualifiés sont soumis, plus que les autres, aux programmes de l'activation; beaucoup ne sont pas comptabilisés comme chômeurs, mais «en congé». Si ces parcours ne débouchent pas sur des emplois stables, la trajectoire d'activation peut devenir pénible, même si elle assure un revenu décent de remplacement. Finalement, les laissés-pour-compte sont surtout les populations immigrantes et, plus particulièrement les femmes, qui connaissent des taux de chômage très élevés et des situations de pauvreté largement plus fréquentes que pour les autres catégories de personnes. Le miracle danois ne semble pas profiter à tous de manière équivalente.

La Grande-Bretagne présente un portrait quasiment opposé en tous points. Si le marché du travail apparaît dynamique et se caractérise par un taux de chômage faible et un taux d'activité élevé, également chez les femmes et les personnes de plus de 55 ans, comme au Danemark, les ressorts de ce dynamisme sont très largement divergents. Cette société de travail pour (presque) tous n'est pas une panacée. Le précariat est en effet très développé parce que beaucoup d'emplois sont à temps partiel (surtout pour les femmes) ou temporaires. Aujourd'hui, presque $30 \%$ des employés n'ont plus de travail à temps plein ou de revenu stable, ce qui crée une masse importante de travailleurs pauvres. Nous avons également noté une croissance importante de l'inégalité des revenus, les hauts salaires ayant connu la plus forte progression, alors que les bas salaires connaissaient la progression la plus faible de ces dernières années. 
De plus, restée fidèle à sa tradition libérale, la GrandeBretagne a limité l'intervention publique en direction des personnes pauvres. Elles sont l'objet d'une forte stigmatisation, les représentations dominantes leur attribuant la responsabilité de la pauvreté et le défaut de la «dépendance» à l'aide publique. Depuis la fin des années 1990, les politiques d'activation des personnes sans emploi n'ont pas fondamentalement modifié cette tendance. L'intervention de l'État demeure ciblée en visant certaines catégories de population (les jeunes, les mères seules), l'aide aux revenus de travail concerne surtout les travailleurs pauvres avec enfants, la législation du travail est très faible et les revenus de remplacement (assistance et assurance) sont particulièrement modestes. Sur l'axe des compromis sociaux, les syndicats sont maintenus en marge de la protection sociale, dont la destinée est l'apanage du gouvernement et de quelques experts de réseaux de politiques. Les personnes handicapées, les parents seuls, les minorités ethniques et les individus sans qualification sont les principaux laissés-pour-compte de la société britannique. De fait, malgré les discours sur l'investissement social et la volonté des gouvernements travaillistes successifs de concilier objectifs sociaux et modernisation économique, la Grande-Bretagne conserve sa place dans le peloton de queue au chapitre de la pauvreté en Europe, et en particulier de la pauvreté des enfants.

On le voit, plusieurs modalités de développement social peuvent soutenir des performances économiques relativement comparables. Dans le cas du Danemark, la collectivité, par le biais de l'État social, prend en charge les «externalités» du capitalisme en assurant les citoyens d'un revenu suffisant. Plus même, l'État intervient avec les partenaires sociaux pour fournir une main-d'œuvre compétitive parce que formée. En Grande-Bretagne, ce sont les individus qui ont la 
responsabilité de voir à ces externalités; la collectivité demeure largement en retrait et l'intervention publique très légère. Ces deux cas représentent des «classiques» de la littérature comparée des États sociaux. Il est intéressant de voir comment on retrouve, sous des formes mixtes, des éléments de ces logiques dans les deux cas hybrides que nous avons traités: la France et le Québec.

En France, les emplois sont accaparés par les 25-54 ans et la plus grande part des performances économiques du pays repose sur ces travailleurs. Aux deux extrêmes de la vie active, on retrouve des taux d'activités très faibles, dus d'une part, à l'allongement des études et aux difficultés pour les jeunes à entrer durablement sur le marché du travail, et d'autre part, à des politiques publiques et des politiques d'embauche qui privilégient le retrait précoce des travailleurs plus âgés par le biais de formules de préretraite. À la différence des autres cas analysés, le chômage est important sur toute la période. Le chômage des jeunes (et des jeunes immigrés ou d'origine étrangère encore plus) est particulièrement préoccupant. La recherche de flexibilité du marché du travail, bien que moins réussie quailleurs, a conduit les pouvoirs publics à créer de nouveaux statuts d'emploi auxquels sont attachés des droits sociaux différenciés.

La dualisation croissante du marché du travail structure fortement la pauvreté: un segment de la main-d'œuvre plus protégé a conservé en grande partie ses droits sociaux et des niveaux de salaires élevés, mais a eu tendance à rester relativement fermé aux nouveaux entrants; alors que la multiplication des statuts d'emploi a produit un fort sentiment d'insécurité pour les travailleurs précaires peu protégés. Les principaux syndicats, faibles numériquement mais forts politiquement, ont participé à ce processus de dualisation, en défendant davantage les droits acquis qu'en faisant la 
promotion de couvertures sociales élargies à l'ensemble des travailleurs, précaires compris. Comme le système des assurances sociales est très lié au statut d'emploi, la segmentation de l'emploi s'accompagne d'une segmentation du traitement politique de la pauvreté. À côté d'un précariat institutionnalisé, composé de travailleurs sous contrats atypiques ou de chômeurs en emplois aidés, les jeunes, les mères seules et les populations immigrées constituent les principales catégories de laissés-pour-compte et connaissent des taux de pauvreté plus importants que les autres.

À la différence de la Grande-Bretagne, les inégalités sociales ont été relativement contenues, sauf si on considère les très hauts revenus qui ont connu une croissance exponentielle dans les cinq dernières années. La France se caractérise par des politiques de redistribution importantes, qui limitent les écarts entre les plus riches et les plus pauvres, et des politiques d'assistance relativement généreuses qui amortissent l'effritement des droits sociaux liées aux changements dans les statuts d'emploi. Comparativement à la Grande-Bretagne, les taux de pauvreté sont beaucoup plus faibles en France; en revanche, la mobilité de la main-d'œuvre est plus difficile et participe à faire du précariat un état plus qu'un passage transitoire. Autrement dit, les précaires français sont - toutes choses étant égales par ailleurs - moins pauvres économiquement que leurs homologues britanniques, mais ils ne jouissent pas d'une sécurité financière aussi étendue et développée que les danois, malgré une forte intervention de l'État.

Au Québec, comme en Grande-Bretagne, la question des travailleurs pauvres est centrale. Elle est liée à la dégradation des conditions de travail et à l'effritement des droits du travail. Moins sévère que dans le cas britannique, la pauvreté des travailleurs existe néanmoins. Dans les dix dernières années, le Québec a profité d'une situation économique 
enviable qui lui a permis de diminuer sensiblement son taux de chômage et de rattraper sur plusieurs indicateurs ses voisins canadiens immédiats, comme la province de l'Ontario. De plus, la lutte contre la pauvreté qui a engagé l'ensemble de la société québécoise a permis une nette amélioration du point de vue de la réduction du taux de pauvreté et un changement significatif des représentations de la pauvreté, perçue aujourd'hui dans sa globalité et non comme une «simple» insuffisance de revenus. Un problème central demeure: les inégalités de revenus, qui sont liées à des politiques de redistribution faibles et des inégalités importantes de salaires, malgré les relèvements récents du salaire minimum.

Engagé, comme les autres sociétés, dans des politiques d'activation de la main-d'œuvre, le Québec n'a pas assorti ces nouveaux programmes sociaux de beaucoup de moyens financiers, ce qui limite grandement la portée des réformes entreprises. Moins interventionniste que le Danemark ou la France, le Québec se démarque néanmoins du cas britannique et des modèles dits libéraux, parce qu'on y retrouve depuis plus de dix ans des acteurs sociaux (syndicats et groupes communautaires) qui tentent de forcer la main du gouvernement dans le champ de la lutte contre la pauvreté et qui proposent aussi des solutions originales pour prendre soin des plus démunis de la société.

L'analyse entreprise ne visait pas à répondre à la question «pourquoi» les sociétés étudiées sont plus ou moins accommodantes vis-à-vis de la pauvreté et des inégalités, comme d'autres le font ${ }^{24}$, mettant de l'avant des facteurs politiques ou institutionnels pour expliquer les trajectoires des pays. L’idée

24. Torben Iversen, John D. Stephens, «Partisan Politics, the Welfare State, and Three Worlds of Human Capital Formation», op. cit. 
initiale consistait plutôt à comprendre d'où viennent les «output» des sociétés en termes de pauvreté et d'inégalités. À chaque cas, nous avons pu faire correspondre des logiques d'articulation des dynamiques politiques, sociales et économiques induisant des formes de pauvreté et d'inégalités.

Pour terminer, nous aimerions insister sur quatre conclusions (partielles) que nous retirons de cette recherche.

1. La mobilité des travailleurs semble une condition du fonctionnement du marché du travail contemporain. Autrement dit, pour tourner, le marché (et les employeurs) a besoin d'une main-d'œuvre mobile, façonnable au gré des besoins de l'économie. Cette mobilité nécessaire peut se transformer en insécurité dans les trajectoires des personnes s'il n'y a pas une prise en charge collective des risques liés à la flexibilité de la main-d'œuvre. L'insécurité sera d'autant plus grande que les marchés du travail comportent des rigidités qui empêchent la circulation d'un emploi à un autre, à la formation, au chômage, etc. Elle peut également se traduire par une relégation pure et simple hors du marché du travail pour des catégories de personnes discriminées ou présentant des profils de formation, de scolarité systématiquement refusés par les employeurs. La flexibilité peut être plus supportable si les transitions sont pensées et assurées et qu'elles ne signifient pas une perte brusque de revenus. Dans cette perspective, les sociétés où le revenu est attaché uniquement ou presque exclusivement à l'emploi apparaissent moins bien armées pour à la fois combattre la pauvreté et être performantes sur le plan économique.

2. La dimension de la négociation collective et des compromis sociaux apparaît centrale dans la possibilité d'entreprendre des réformes d'envergure et de transformer durablement les dynamiques qui sous-tendent la production de la pauvreté et des inégalités. Autant au Danemark avec l'inclusion 
des acteurs collectifs et patronaux à la gestion du marché du travail qu'au Québec avec la participation des acteurs sociaux à la gestion de la pauvreté, le compromis social apparaît comme une clef pour atteindre des objectifs de développement social. La France et la Grande-Bretagne auront probablement besoin de toutes leurs forces vives (collectives) pour lutter contre la pauvreté endémique (Grande-Bretagne) ou renverser le développement d'un précariat institutionnalisé (France).

3. La lutte contre la pauvreté et la réduction des inégalités constituent des choix collectifs, des choix de société, qui peuvent se traduire par des actions politiques concrètes. Il n'y a pas de fatalité à ce chapitre, ni de convergence mécanique. La destinée des sociétés repose entre les mains des acteurs qui les composent. Néanmoins, nous avons noté une tension croissante entre les reconfigurations du monde du travail et de la recherche d'emploi (injonctions à l'individualisation, à la responsabilisation des individus et à l'émiettement des statuts collectifs) et la nécessité de la prise en charge collective de la question sociale.

4. Dans les quatre sociétés analysées, ce sont les personnes immigrantes qui sont les plus mal loties. C'est vrai du chômage, des taux de pauvreté, de l'exclusion plus durable. Le futur pourra difficilement se faire sans elles; leur inclusion véritable appelle des changements profonds autant sur le plan des représentations sociales que des politiques et des comportements individuels et collectifs. L'histoire des démocraties du Nord, et en particulier les années post-crise de 1929, nous a appris que chercher à souder la société par une dynamique de division entre des inclus et des «autres», en utilisant les étrangers de l'intérieur comme variable d'ajustement, voire comme bouc-émissaire, ne peut être qu'une défaite collective. 



\section{BIBLIOGRAPHIE}

\section{Ouvrages généraux}

Alcock, Pete, Social Policy in Britain, Palgrave-Macmillan, 2003.

Alesina, Alberto et Edward L. Glaeser, Combattre les inégalités et la pauvreté. Les États-Unis face à l'Europe, Flammarion, 2004.

Amparo Serrano, Pascual et Lars Magnusson (dir.), Reshaping Welfare States and Activation Regimes in Europe, Peter Lang, 2007.

Andersen, Torben M. et Michael Svarer, Flexicurity - Labour Market Performance in Denmark, University of Aarhus, 2007.

Atkinson, Anthony B. et Thomas Piketty (dir.), Top Incomes over the Twentieth Century, A Contrast Between European and English-Speaking Countries, Oxford University Press, 2007.

Auerbach, Alan J., David E. Card et John M. Quigley (dir.), Public Policy and the Income Distribution, Russell Sage Foundation, 2006.

Barbier, Jean-Claude, La longue marche vers l'Europe sociale, Presses universitaires de France, 2009.

Barbier, Jean-Claude et Bruno Théret, Le nouveau système français de protection sociale, La Découverte, 2004.

Barnes, Matt, Social Exclusion in Great Britain: An Empirical Investigation and Comparison with the EU, Ashgate, 2005.

Bec, Colette, Giovanna Procacci et Claudine Attias-Donfut (dir.), De la responsabilité solidaire: mutations dans les politiques sociales aujourd'hui, Syllepse, 2003.

Bernier, Nicole F., Le désengagement de l'État-providence, Presses de l'Université de Montréal, 2003.

Boltanski, Luc, De la critique. Précis de sociologie de l'émancipation, Gallimard, 2009.

Bourque, Gilles L., Le modèle québécois de développement. De l'émergence au renouvellement, Presses de l'Université du Québec, 2000. 
Boychuk, Gerard W., Patchwork of Purposes: The Development of Provincial Social Assistance Regimes in Canada, McGill-Queen's University Press, 1998.

Bruno, Isabelle, «Déchiffrer l'“Europe compétitive”. Étude du benchmarking comme technique de coordination intergouvernementale dans le cadre de la stratégie de Lisbonne», Thèse de doctorat, IEP de Paris, 2006.

Campbell, John L., John A. Hall et Ove Kaj Pedersen (dir.), National Identity and the Varieties of Capitalism: The Danish Experience, McGillQueen's University Press, 2006.

Castel, Robert, L'insécurité sociale: Qu'est-ce qu'être protégé?, Seuil, 2003.

Castel, Robert, La montée des incertitudes. Travail, protections, statut de l'individu, Seuil, 2009.

Castles, F. G. (dir.), Families of Nations: Patterns of Public Policy in Western Democracies, Darmouth, 1993, p. 93-127.

Clasen, Jochen, Reforming European Welfare States: Germany and the United Kingdom Compared, Oxford University Press, 2005.

Cohen, Antonin, Bernard Lacroix et Philippe Riutort (dir.), Les formes de l'activité politique. Eléments d'analyse sociologique, $X V I I I^{e}-X X^{e}$ siècle, Presses universitaires de France, 2006.

Daguerre, Anne et Trine P. Larsen, Low Pay and Social Assistance: Minimum Wage and Tax Credits, University of Kent, 2001.

Daguerre, Anne et Trine P. Larsen, The 1996 Jobseeker's Allowance Act (JSA), University of Kent, 2001.

Danziger, Sheldon et Robert H. Haveman (dir.), Understanding Poverty, Russell Sage Foundation et Harvard University Press, 2001.

Data, Lorraine, Le grand truquage. Comment le gouvernement manipule les statistiques, La Découverte, 2009.

Denis, Roch et Serge Denis, Les syndicats face au pouvoir. Syndicalisme et politique au Québec de 1960 à 1992, Vermillon, 1992.

Desrosières, A., La politique des grands nombres, histoire de la raison statistique, La Découverte, 1993.

Didier, Emmanuel, En quoi consiste l'Amérique? Les statistiques, le New Deal et la démocratie, La Découverte, 2009.

Dobrowolsky, Alexandra et Ruth Lister, Social Exclusion and Changes to Citizenship: Woman and Children, Minorities and Migrants in Britain, University of Western, 2005.

Dufour, Pascale, Gérard Boismenu et Alain Noël, L'aide au conditionnel: la contrepartie dans les mesures envers les personnes sans emploi en Europe et en Amérique du Nord, Presses de l'Université de Montréal et Peter Lang, 2003.

Duvoux, Nicolas et Serge Paugam, La régulation des pauvres, Presses universitaires de France, 2008. 
Ehrenreich, Barbara, L’Amérique pauvre, Éditions 10/18, 2004.

Elague, Michael et Brian Wharf (dir.), Community Organizing Canadian Experiences, Oxford University Press, 1997.

Esping-Andersen, Gøsta (dir.), Welfare States in Transition: National Adaptation in Global Economies, Sage Publications, 1996.

Esping-Andersen, Gøsta, The Three Worlds of Welfare Capitalism, Princeton University Press, 1990.

Esping-Andersen, Gøsta, Trois leçons sur l'État-providence, Seuil, 2008.

Faucher-King, Florence et Patrick Le Galès, Tony Blair 1997-2007, Le bilan des réformes, Presses de Sciences Po, 2007.

Fourquet, F., Les comptes de la puissance, Encres, 1980.

Fremstad, Shawn et al., Movin' On Up: Reforming America's Social Contract to Provide a Bridge to the Middle Class, Centre for Economic and Policy Research, 2008.

Gadrey, Jean et Florence Jany-Catrice, Les nouveaux indicateurs de richesse, La Découverte, 2005.

Gagnon, Alain G. (dir.), Quebec: State and Society, Broadview Press, 2004, p. 427-446.

Gazier, Bernard, Tous sublimes, vers un nouveau plein emploi, Flammarion, 2003.

Giddens, Anthony, The Third Way. The Renewal of Social Democracy, Polity Press, 1998.

Hall, Peter et David Soskice (dir.), Varieties of Capitalism, Oxford University Press, 2001.

Hills, John et Kitty Stewart (dir.), A More Equal Society? New Labour, Poverty, Inequality and Exclusion, Policy Press, 2005.

Hills, John, Inequality and the State, Oxford University Press, 2004.

Hoang, Ngoc Liêm, Sous la crise, la répartition des revenus, La dispute, 2009.

Jenson, Jane, Bérengère Marques-Pereira et Éric Remacle (dir.), L'état des citoyennetés en Europe et dans les Amériques, Presses de l'Université de Montréal, 2007.

Katzenstein, Peter J., Small States in World Makets, Cornell University Press, 1985.

Kober, Claire et Will Paxton (dir.), Asset-based Welfare and Poverty: Exploring the Case for and against Asset-based Welfare Policies, National Children's Bureau Enterprises, 2002.

L'Horty, Yannick, Revenu minimum et retour à l'emploi: une perspective européenne?, Université d'Evry, 2004.

Laffut, Michel et Marie-Renée Roy, Pauvreté et exclusion sociale: partage d'expériences entre Wallonie et Québec, De Boeck, 2007. 
Larsen, Trine P., Anne Daguerre et Peter Taylor-Gooby, Woman and the Labour Market - The National Childcare Strategy Including Sure Start and the Integrated Child Credit, University of Kent, 2001.

Lind Jens, Herman Knudsen et Henning Jørgensen (dir.), Labour and Employment Regulation in Europe, Peter Lang, 2004.

Lødemel, Ivar et Heather Trickey (dir.), «An Offer You Can't Refuse»: Workfare in International Perspective, Policy Press, 2001.

Ludlam, Steve et Martin J. Smith (dir.), Governing as New Labour. Policy and Politics under Blair, Macmillan, 2004.

Matt Barnes et al. (dir.), Poverty and Social Exclusion in Europe, Edward Elgar Pub, 2002.

Maurin, Éric, La peur du déclassement, une sociologie des récessions, Seuil, 2009.

Millar, Jane (dir.), Understanding Social Security: Issues for Policy and Practice, Policy Press, 2003.

Noël, Alain, Federalism and Labour Market Policy: Comparing Different Governance and Employment Strategies, McGill-Queen's University Press, 2004.

Palier, Bruno, Gouverner la sécurité sociale: Les réformes du système français de protection sociale depuis 1945, Quadrige/Presses universitaires de France, 2005.

Palier, Bruno et Christelle Mandin, Policy Maps - France, University of Kent, 2003,

Palmer, Guy, Tom MacInnes et Peter Kenway, Monitoring Poverty and Social Exclusion 2006, New Policy Institute, 2006.

Paugam, Serge, Les formes élémentaires de la pauvreté, Presses universitaires de France, 2005.

Paugam, Serge (dir.), Repenser la solidarité. L'apport des sciences sociales, Presses universitaires de France, 2007.

Pierson, Paul, Dismantling the Welfare State? Reagan, Thatcher and the Politics of Retrenchment, Cambridge University Press, 1995.

Pierson, Paul (dir.), The New Politics of the Welfare State, Oxford University Press, 2001.

Pontusson, Jonas, Inequality and Prosperity: Social Europe vs. Liberal America, Cornell University Press, 2005.

Richard G. Harris (dir.), North American Linkages: Opportunities and Challenges for Canada, University of Calgary Press, 2004.

Rowntree, Seebohm, Poverty and Progress, Longmans Green, 1941.

Rowntree, Seebohm, Poverty: A Study of Town Life, Nelson, 1901.

Roy, Marie-Renée, Guy Fréchet et Frédéric Savard, Travail et pauvreté au Canada, Options politiques, IRPP, 2008. 
Rueschmeyer, Dietrich et Theda Skocpol, States, Social Knowledge, and the Origins of Modern Social Policies, Princeton University Press, Russell Sage Foundation, 1996.

Sainsbury, D. (dir.), Gendering Welfare States, Sage Publications, 1994.

Smeeding, Timoty S. et Lee Rainwater, Comparing Living Standards across Nations: Real Incomes at the Top, the Bottom, and the Middle, University of New South Wales, $2 \mathrm{OO} 2$.

Sutherland, Holly, Tom Shefton et David Piachaud, Poverty in Britain: The Impact of Government Policy since 1997, Joseph Rowntree Foundation, 2003.

Townsend, Peter, Poverty in the United Kingdom, a Survey of Household Resources and Standards of Living, Penguin Books \& Allen Lane, 1979.

Ulysse, Pierre-Joseph et Frédéric Lesemann, Citoyenneté et pauvreté: politiques, pratiques et stratégies d'insertion en emploi et de lutte contre la pauvreté, Presses de l'Université du Québec, 2004.

Vanoli, A., Une histoire de la comptabilité nationale, La Découverte, 2002.

Vosko, Leah, Precarious Employment. Understanding Labour Market Insecurity in Canada, McGill-Queen's University Press, 2006.

Yvon, Leclerc et Claude Béland (dir.), La voie citoyenne: pour renouveler le modèle québécois, Plurimédia, 2003.

Zajdela, Hélène et Ai-Thu Dang, Fondements normatifs des politiques d'activation: un éclairage à partir des théories de la justice, Centre d'étude de l'emploi, 2007.

Zimmermann, Bénédicte (dir.), Les sciences sociales à l'épreuve de l'action. Le savant, le politique et l'Europe, Éditions de la Maison des sciences de l'homme, 2004.

\section{Sources administratives et gouvernementales}

Arloc, Sherman, Income Inequality Hits Record Levels, New CBO Data Show Incomes Rose \$180,000 for Top One Percent in 2005. But Just $\$ 400$ for Middle-Income Households, Center on Budget and Policy Priorities, 2007.

Barbier, Jean-Claude (dir.), Analyse comparative de l'activation de la protection sociale en France, Grande-Bretagne, Allemagne et Danemark, dans le cadre des lignes directrices de la stratégie européenne pour l'emploi, DARES, Ministère du Travail, 2006.

Bélanger, Paul R. et Benoît Lévesque, Le modèle québécois: corporatisme ou démocratie sociale?, CRISES/UQAM, 2001.

Bredgaard, Thomas, Larsen Flemming et Per Kongshøj Madsen, The Flexible Danish Labour Market - A Review, Aalborg University, 2005. 
Bredgaard, Thomas et Larsen Flemming, The Transitional Danish Labour Market: Understanding a Best Case, and Policy Proposals for Solving Some Paradoxes, CARMA, Aalborg University, Research Paper, 2006.

Centre d'étude sur la pauvreté et l'exclusion, Prendre la mesure de la pauvreté. Proposition d'indicateurs de pauvreté, d'inégalités et d'exclusion sociale afin de mesurer les progrès réalisés au Québec. Avis au ministre, 2009.

Chaykowski, Richard P., Non-Standard Work and Economic Vulnerability: Defining Vulnerability in the Labour Market, Réseaux canadiens de recherche en politiques publiques, 2005.

Collectif pour un Québec sans pauvreté, «Déclaration citoyenne: pour une sécurité du revenu couvrant les besoins dans la dignité et les droits», communiqué de presse, 8 décembre 2004.

Colloque sur la loi 112 du Collectif pour un Québec sans pauvreté, L'horizon d'un Québec sans pauvreté versus la Loi visant à lutter contre la pauvreté et l'exclusion sociale: un processus et ses questions, 2005.

Conseil européen, "Conclusions de la Présidence», Sommet européen de Lisbonne, 23 et 24 mars 2000, Nr: 100/1/OO, §5.

Cooke, Graeme et Kayte Lawton, Working out of Poverty. A Study of the Low-paid and the "Working Poor», Institute for Public Policy Research, 2008.

Daguerre, Anne et Trine P. Larsen, Low Pay and Social Assistance: Minimum Wage and Tax Credits, University of Kent.

Daguerre, Anne et Trine P. Larsen, The 1996 Jobseeker's Allowance Act (JSA), University of Kent.

Danemark, National Report on Strategies for Social Protection and Social Inclusion, Ministry of Social Affairs et Ministry of Interior Affairs and Health, 2006.

Egger, Philippe et Werner Sengenberger (dir.), Decent Work in Denmark: Employment, Social Efficiency and Economic Security, International Labour Office, 2003.

Front commun des personnes assistées sociales du Québec (FCPASQ), Lutte à la pauvreté. Bilan des 100 premiers jours du gouvernement Charest, conférence de Jean-Yves Desgagnés, 2003.

Gouvernement du Québec, Comité consultatif de lutte contre la pauvreté et l'exclusion sociale. Planification et orientations 2006-2009, 2007.

Gouvernement du Québec, Inventaire des indicateurs de pauvreté et d'exclusion sociale. Conditions de vie, 2005.

Gouvernement du Québec, Le faible revenu au Québec: un état de la situation, Centre d'étude de la pauvreté et de l'exclusion, 2008, p. 4.

Grivel, Nicolas, Nathalie Georges et Dominique Méda, Les prestations et services d'accompagnement des demandeurs d'emploi: comparaisons internationales Suède, Pays-Bas, Royaume-Uni, Centre d'étude de l'emploi, 2007. 
Harker, Lisa, Delivering on Child Poverty: What Would It Take?, Secretary of State for Work and Pensions, 2006.

Jacobsson, Kerstin, «Achievements and Non-Achievements of the European Employment Strategy», Morel, Nathalie, Bruno Palier et Joakim Palme, What Future for Social Investment?, Institute for Futures Studies, Research Report, 2009, p. 119-130.

Jenson, Jane, «Redesigning Citizenship Regimes after Neoliberalism. Moving Towards Social Investment», Morel, Nathalie, Bruno Palier et Joakim Palme, What Future for Social Investment?, Institute for Futures Studies Research Report, 2009, p. 27-44.

Kemp, Peter, Jonathan Bradshaw, Paul Dornan, Naomi Finch et Emese Mayhew, Routes out of Poverty. A Research Review, Joseph Rowntree Foundation, 2004.

Labrie, Viviane, «Projet de loi 57 réformant l'aide sociale: le Collectif exige le retrait du projet de loi 57 et propose des solutions pour améliorer le régime d'aide sociale», communiqué de presse, 2004.

Larsen, Trine P., Anne Daguerre et Peter Taylor-Gooby, Woman and the Labour Market - The National Childcare Strategy Including Sure Start and the Integrated Child Credit, University of Kent, 2001.

Madsen, Per Kongshøj, Danish Labour Market Institutions and Employment Outcomes, Federal Ministry of Economics and Technology, 2007.

Michaud, Mélanie, France Gagnon et Joël Gauthier, Le plan d'action gouvernemental en matière de lutte contre la pauvreté et l'exclusion sociale. Étude de cas - Résumé, Groupe d'étude sur les politiques publiques et la santé, 2006.

Morissette, René et Garnett Picot, Le travail peu rémunéré et les familles économiquement vulnérables depuis 20 ans, Rapport de recherche de la Direction des études analytiques, Statistique Canada, 2005.

Nikolai, Rita, «Towards Social Investment? Patterns of Public Policy in the OECD World», Morel, Nathalie, Bruno Palier et Joakim Palme (dir.), What Future for Social Investment?, Institute for Futures Studies Research Report, 2009, p. 101-102.

Noël, Alain, Priorité au soutien du revenu: La mise en œuvre de la Loi québécoise visant à lutter contre la pauvreté et l'exclusion sociale, Canadian Policy Research Networks, 2004, p. 1-16.

OCDE, «Croissance et inégalités: distribution des revenus et pauvreté dans les pays de l'OCDE», 2008.

OCDE, Earnings Inequality, Low-paid Employment and Earning Mobility, Employment Outlook, 1996.

OCDE, Perspectives de l'emploi de l'OCDE. Stimuler l'emploi et les revenus, Organisation de coopération et de développement économiques, 2006. 
Osberg, Lars et Andrew Sharpe, «Measuring Economic Security in Insecure Times: New Perspectives, New Events, and the Index of Economic Well-being», CSLS Report, 2009.

Osberg, Lars et Andrew Sharpe, «New Estimates of the Index of Economic Well-being for Selected OECD Countries, 1980-2007», CSLS Report, 2009.

Paquet, Gilles, «Québec Inc. : Mythes et réalités», Filip Palda (dir.), L'État interventionniste: le gouvernement provincial et l'économie du Québec, Fraser Institute, 1994.

Québec, Assemblée nationale, Loi visant à lutter contre la pauvreté et l'exclusion sociale, $36^{\mathrm{e}}$ législature, deuxième session, Projet de loi $\mathrm{n}^{\circ}$ 112, 2002.

Royaume-Uni, UK National Report on Strategies for Social Protection and Social Inclusion 2006-2008, Ministry of Labour and Social Affairs, 2006.

Royaume-Uni. Opportunity for All - Eighth Annual Report 2006: Strategy Document, Department for Work and Pensions, 2006.

Sarlo, Chris, What is Poverty? Providing Clarity for Canada, Fraser Institute, 2008.

Saunders, Ron, Risques et possibilités: à la recherche d'options pour les travailleurs vulnérables, Canadian Policy Research Networks, 2006, p. 9.

Statistique Canada, Le revenu au Canada 2005, Ottawa, mai 2007.

Stiglitz, Joseph, Amartya Sen et Jean-Paul Fitoussi, Rapport de la Commission sur la mesure des performances économiques et du progrès social, Ministère de l'Économie, de l'Industrie et de l'Emploi, septembre 2009.

Ulysse, Pierre-Joseph, Travail et pauvreté. Quand le travail n'empêche plus d'être pauvre!, Forum régional de développement social de l'île de Montréal, 2006.

\section{Périodiques}

Actes de la recherche en sciences sociales

American Political Science Review

American Sociological Review

Canadian Journal of Sociology/Cahiers canadiens de sociologie

Centre d'études de l'emploi

Chronique internationale de l'IRES

Comparative Political Studies

Comparative Social Research

Critique internationale

Droit Social 
Économie et statistique

Environment and Planning C: Government and Policy

Études internationales

European Journal of Industrial Relations

European Review of Labour and Research

European Societies

Genèses

Industrial Relations Journal

Informations sociales

International Journal of Sociology and Social Policy

International Social Security Review

Journal of Commonwealth and Comparative Politics

Journal of European Public Policy

Journal of Social Policy

Journal of Social Sciences

L'Observatoire de l'ANPE: l'essentiel

La Revue de l'IRES

Les Politiques sociales

Lien social et politiques

Monthly Labour Review

Nouvelles pratiques sociales

Oxford Review of Economic Policy

Perceptions

Perspectives on Labour and Income

Politiques et Sociétés

Politix - Revue des sciences sociales du politique

Recherches et Prévisions

Recherches sociographiques

Regards croisés sur l'économie

Regulation \& Governance

Relations industrielles

Revue canadienne de science politique

Revue d'économie politique

Revue de droit sanitaire et social

Revue économique

Revue française de science politique

Revue française de sociologie

Revue française des affaires sociales

Revue Internationale de Politique Comparée 
Savoir/Agir, Revue trimestrielle de l'association Savoir/agir

Social Policy \& Administration

Sociologie et sociétés

The Industrial Law Journal

Theory, Culture and Society

Vives lectures

\section{Références web}

BIP4O, Baromètre des inégalités et de la pauvreté, www.bip40.org

Canada, Service Canada, Loi sur l'assurance-emploi, Partie I, Prestations de chômage, www1.servicecanada.gc.ca/fr/ae/legislation/ae_lois_ partie1_7.shtml\#7_1

CSLS, Centre for the Study of Living Standards, www.csls.ca

Gouvernement du Québec, Crédits et effectifs 2006-2007 du ministère de l'Emploi et de la Solidarité sociale, Emploi et Solidarité sociale, www. mess.gouv.qc.ca/ministere/credits-effectifs/credits-effectifs2Oo62007.asp

Institut de la statistique du Québec, Coefficient de Gini calculé selon les concepts de revenu différents, 1996 à 2007, www.stat.gouv.qc.ca/donstat/ societe/famls_mengs_niv_vie/revenus_depense/revenus/ginitab96 _2007.htm

Institut de la statistique du Québec, Coefficient de Gini et contributions des composantes de revenu, toutes les familles, 1979-2004, www.stat.gouv. qc.ca/publications/conditions/pdf2007/InegaliteRevenu.pdf

Institut de la statistique du Québec, Inventaire des indicateurs de pauvreté et d'exclusion sociale, Institut de la statistique du Québec et Ministère de l'Emploi et de la Solidarité, 2005.

Institut de la statistique du Québec, PIB aux prix du marché par habitant, 20O2-2O11, www.stat.gouv.qc.ca/donstat/econm_finnc/conjn_econm/ TSC/index.htm

Institut de statistique du Québec, Rémunération horaire moyenne des employés selon diverses caractéristiques, moyennes annuelles, Québec, 1997 à 2007, www.stat.gouv.qc.ca/donstat/societe/march_travl_remnr/ remnr_condt_travl/coo1_rem_hor_emp_9707.htm

Institut de la statistique du Québec, Taux de chômage selon le groupe d'âge et selon le sexe, moyennes annuelles, Québec, 1976 à 2007, www.stat.gouv. qc.ca/donstat/societe/march_travl_remnr/parnt_etudn_march_ travl/pop_active/tab17.htm

Institut de la statistique du Québec, Taux de faibles revenus. Éditions 2008. www.stat.gouv.qc.ca/publications/regions/flash_faible_revenu_pdf. htm 
Institut de la statistique du Québec, Taux de salaire minimum sur une base horaire pour les travailleurs adultes, www.stat.gouv.qc.ca/donstat/ societe/march_travl_remnr/remnr_condt_travl/eoo1_taux_hor_sal _min_janv97_maio8.htm

Institut pour le développement de l'information économique et sociale, FAIR, www.idies.org/index.php?category/FAIR

Morel, Sylvie, Modèle du workfare ou modèle de l'insertion? La transformation de l'assistance sociale au Canada et au Québec, Condition féminine Canada, dsp-psd.tpsgc.gc.ca/Collection/SW21-95-2002F.pdf

Picot, Garnett et Hou Feng, The Rise in Low Income Rates among Immigrants in Canada, Statistique Canada, www.statcan.gc.ca/bsolc/olc-cel/ olc-cel?catno $=11$ Foo $19 \mathrm{M} 2003198=\mathrm{eng}$

Québec, Le pacte pour l'emploi. Le Québec de toutes ses forces, www.pacteemploi.gouv.qc.ca/presentation/index.asp

Québec, Répertoire des programmes: Services aux citoyens. Portail Québec, Services Québec, www.formulaire.gouv.gc.ca

Statistique Canada, Bénéficiaires d'assurance-emploi selon la province, www. statcan.ca/francais/freepub/71-222-XIF/200400o/chart-k62_f.htm

Statistique Canada, Évolution de la rémunération horaire moyenne des salariés rémunérés à l'heure selon la branche d'activité, 2003-2007, www40. statcan.ca/lo2/csto1/labr74a_f.htm 



\section{TABLE DES MATIÈRES}

AVANT-PROPOS

1 Comprendre la pauvreté: enjeux politiques et analytiques

La permanence de la pauvreté

Transformations des trajectoires de l'État social

Prendre la mesure de la pauvreté

Comparer les fondements de la pauvreté

2 Les arêtes du triangle danois

Un marché du travail flexible et dynamique,

une population très active

Une activation reposant sur le développement

de l'employabilité des travailleurs

Le traitement politique de la pauvreté: l'universalisme et le primat de l'inclusion comme matrice sociale

Régulation du marché du travail

et maîtrise des antagonismes sociaux

Les revers de l'ambition universaliste

3 La légitimité des inégalités et la question sociale en Grande-Bretagne

Un marché du travail actif après de grandes difficultés

La stigmatisation de la pauvreté

et la responsabilisation des individus

Le travail comme pivot de la protection sociale

La faiblesse du tripartisme

et la marginalisation des syndicats

Pauvreté illégitime, inégalités légitimes? 
4 L'idéal solidariste républicain français face à la dualisation du marché du travail

La pesanteur du chômage de masse $\quad 90$

La monopolisation par l'État de l'intérêt général 94

Intolérance (théorique) et accommodements

(pratiques) envers la pauvreté $\quad 97$

La dualisation du marché du travail:

vers un workfare à la française? 102

D’une assise égalitaire à la montée en force récente $\begin{array}{ll}\text { du précariat et des working rich } & 109\end{array}$

5 La lutte québécoise contre la pauvreté dans un contexte inégalitaire $\quad 117$

Une situation fragile $\quad 119$

Un marché du travail fragilisé $\quad 123$

Des mesures et dispositifs de protection sociale

qui soulagent $\quad 127$

Une société mobilisée par des enjeux de pauvreté $\quad 130$

À la manière du Québec $\quad 137$

Les laissés-pour-compte $\quad 142$

Conclusion 146

6 (Dé)chiffrer la pauvreté: des instruments d'objectivation en débats 151

La faute aux indicateurs? Enjeux de la quantification $\quad 151$

Mesure de la pauvreté et pauvreté de la mesure $\quad 155$

L'objectivation statistique du social

à l'épreuve du benchmarking politique 166

7 Vous dites convergence? 173

Une convergence qui se remarque $\quad 173$

Dissonances ou affirmation de modèles $\quad 175$

Différenciation et complexité sociales $\quad 178$

L'innovation plutôt que la fatalité $\quad 180$

Une démarche multidimensionnelle $\quad 183$

Des tendances de fond $\quad 185$

La logique des articulations $\quad 190$

$\begin{array}{ll}\text { BIBLIOGRAPHIE } & 199\end{array}$ 


\section{DANS LA MÊME COLLECTION}

Comité spécial du Sénat sur les drogues illicites, Le cannabis. Rapport du Comité spécial du Sénat sur les drogues illicites

Normand Baillargeon, Contre la réforme. La dérive idéologique du sytème d'éducation québécois

Charles Blattberg, Et si nous dansions? Pour une politique du bien commun au Canada

Gérard Boismenu et Guylaine Beaudry, Le nouveau monde numérique. Le cas des revues universitaires

Esther Cohen, Les narrateurs d'Auschwitz

Yolande Cohen, Femmes philanthropes. Catholiques, protestantes et juives dans les organisations caritatives au Québec

Joseph Heath, La société efficiente. Pourquoi fait-il si bon vivre au Canada?

Othmar Keel, La médecine des preuves. Une histoire de l'expérimentation thérapeutique par essais cliniques contrôlés

Ingo Kolboom, Pièces d'identité. Signets d'une décennie allemande 1989-2000

Armand Mattelart, Pour un regard-monde. Entretiens avec Michel Sénécal

Catherine Mavrikakis, Condamner à mort. Les meurtres et la loi à l'écran

Éric Méchoulan, La culture de la mémoire ou comment se débarrasser du passé?

Alain Noël et Jean-Philippe Thérien, La gauche et la droite. Un débat sans frontières 
